\title{
Toward Resolution of Singularities over a Field of Positive Characteristic
}

\author{
Dedicated to Professor Heisuke Hironaka \\ Part I. \\ Foundation; the language of the idealistic filtration
}

By

\author{
Hiraku KaWANOUE*
}

\section{Contents}

Chapter 0. Introduction

$\S 0.1$. Goal of this series of papers

$\S 0.2$. Overview of the program

0.2.1. Crash course on the existing algorithm(s) in characteristic zero.

0.2.2. Trouble in positive characteristic.

0.2.3. Our program: a new approach in the framework of the idealistic filtration.

$\S 0.3$. Algorithm constructed according to the program

0.3.1. Algorithm in characteristic zero.

0.3.2. Algorithm in positive characteristic; the remaining problem of termination.

§0.4. Assumption on the base field

0.4.1. Perfect case.

0.4.2. Non-perfect case.

$\S 0.5$. Other methods and approaches

0.5.1. Brief history.

0.5.2. Recent announcements of other new approaches.

Communicated by S. Mori. Received August 2, 2006. Revised March 23, 2007. 2000 Mathematics Subject Classification(s): 14E15.

* Research Institute for Mathematical Sciences, Kyoto University, Oiwake-cho, Kitashirakawa, Sakyo-ku, Kyoto 606-8502, Japan.

e-mail: kawanoue@kurims.kyoto-u.ac.jp

(c) 2007 Research Institute for Mathematical Sciences, Kyoto University. All rights reserved. 
$\S 0.6$. Origin of our program and its name

$\S 0.7$. Acknowledgement

$\S 0.8$. Outline of Part I

$\S 0.9$. Preliminaries

0.9.1. The language of schemes.

0.9.2. Basic facts from commutative algebra.

0.9.3. Multi-index notation.

Chapter 1. Basics on Differential Operators

$\S 1.1$. Definitions and first properties

1.1.1. Definitions.

1.1.2. First properties.

$\S 1.2$. Basic properties of differential operators on a variety smooth over $k$

1.2.1. Explicit description of differential operators with respect to a regular system of parameters.

1.2.2. Logarithmic differential operators.

1.2.3. Relation with multiplicity.

$\S 1.3$. Ideals generated by the $p^{e}$-th power elements

1.3.1. Characterization in terms of the differential operators.

Chapter 2. Idealistic Filtration

$\S 2.1$. Idealistic filtration over a ring

2.1.1. Definitions.

2.1.2. D-saturation.

2.1.3. R-saturation.

2.1.4. Integral closure.

2.1.5. $\mathfrak{B}$-saturation.

$\S 2.2$. Basic properties of an idealistic filtration

2.2.1. On generation, $\mathfrak{D}$-saturation, $\mathfrak{R}$-saturation, integral closure, and $\mathfrak{B}$-saturation.

2.2.2. $\mathfrak{R}$-saturated implies integrally closed.

2.2.3. Analysis of interaction between $\mathfrak{D}$-saturation and $\mathfrak{R}$-saturation.

§2.3. Idealistic filtration of r.f.g. type

2.3.1. Stability of r.f.g. type under $\mathfrak{D}$-saturation.

2.3.2. Stability under $\mathfrak{R}$-saturation.

$\S 2.4$. Localization and completion of an idealistic filtration

2.4.1. Definition.

2.4.2. Compatibility. 
Chapter 3. Leading Generator System

§3.1. Analysis of the leading terms of an idealistic filtration

3.1.1. Definitions.

3.1.2. Heart of our analysis.

3.1.3. Leading generator system.

$\S 3.2$. Invariants $\sigma$ and $\widetilde{\mu}$

3.2.1. Invariant $\sigma$.

3.2.2. Invariant $\widetilde{\mu}$.

Chapter 4. Nonsingularity Principle

$\S 4.1$. Preparation toward the nonsingularity principle

4.1.1. Setting for the supporting lemmas.

4.1.2. Statements and proofs of the supporting lemmas.

4.1.3. Setting for the coefficient lemma.

4.1.4. Statement and proof of the coefficient lemma.

$\S 4.2$. Nonsingularity principle

4.2.1. Statement of the nonsingularity principle.

4.2.2. Proof of the nonsingularity principle.

References

\section{Chapter 0. Introduction}

\section{$\S 0.1$. Goal of this series of papers}

This is the first of the series of papers under the title

"Toward resolution of singularities over a field of positive characteristic"

Part I. Foundation; the language of the idealistic filtration

Part II. Basic invariants associated to the idealistic filtration and their properties

Part III. Transformations and modifications of the idealistic filtration

Part IV. Algorithm in the framework of the idealistic filtration

Our goal is to present a program toward constructing an algorithm for resolution of singularities of an algebraic variety over a perfect field $k$ of positive characteristic $p=\operatorname{char}(k)>0$. We would like to emphasize, however, that the program is created in the spirit of developing a uniform point of view toward the problem of resolution of singularities in all characteristics, and hence that it is also valid in characteristic zero. 
In Part I, we establish the notion and some fundamental properties of an idealistic filtration, which is the main language to describe the program. This part, therefore, forms the foundation of the program.

In Part II, we study the basic invariants $\sigma$ and $\widetilde{\mu}$ associated to an idealistic filtration, which will become the building blocks toward constructing the strand of invariants used in our algorithm, and discuss their properties.

In Part III, we analyze the behavior of an idealistic filtration under the two main operations in the process of our algorithm for resolution of singularities:

- transformations of an idealistic filtration under the operation of taking blowups, and

- modifications of an idealistic filtration under the operation of constructing the strand of invariants.

Part II and Part III should play the role of a bridge between the foundation in Part I and the presentation of our algorithm in Part IV.

In Part IV, we present our algorithm for resolution of singularities according to the program as a summary of the series. In characteristic zero, the program leads to a complete algorithm (slightly different from the existing ones), which then serves as a prototype toward the case in positive characteristic. In positive characteristic, all the ingredients of the program work nicely forming a perfect parallel to the case in characteristic zero, except for the problem of termination: we do not know at this point whether our algorithm terminates after finitely many steps or not. Although we do know that the strand of invariants we construct strictly drops after each blowup, we can not exclude the possibility that the denominators of some invariants in the strand may indefinitely increase and hence that the descending chain condition may not be satisfied. The problem of termination remains as the only missing piece toward completing our algorithm in positive characteristic. We hope, however, that we may be able to fix this problem during the process of writing down all the details of the program in this series of papers.

\section{§0.2. Overview of the program}

Below we present an overview of the program, by first giving a crash course on the existing algorithm(s) in characteristic zero, then pinpointing the main source of troubles if we try to apply the same methods to the case in positive characteristic, and finally describing how our program attempts to overcome these troubles. 


\subsubsection{Crash course on the existing algorithm(s) in characteristic} zero.

0.2.1.1. Standard reduction. By a standard argument free of characteristic, the problem of resolution of singularities of an abstract algebraic variety is reduced to, and reformulated as, the problem of transforming a given ideal $\mathcal{I} \subset \mathcal{O}_{W}$ on a nonsingular variety $W$ over $k$ into the one whose multiplicity (order) becomes lower than the aimed (or expected) multiplicity $a$ everywhere, through a sequence of blowups and through a certain transformation rule for the ideal. We require that each center of blowup to be nonsingular and transversal to the boundary, which consists of the exceptional divisor and the strict transform of a simple normal crossing divisor $E$ on $W$ given at the beginning. We call this reformulation the problem of resolution of singularities of the triplet $(W,(\mathcal{I}, a), E)$, and call $\operatorname{Sing}(\mathcal{I}, a)=\left\{P \in W ; \operatorname{ord}_{P}(\mathcal{I}) \geq a\right\}$ its singular locus or support.

0.2.1.2. Inductive scheme in characteristic zero. At the very core of all the existing algorithmic approaches in characteristic zero lies the common inductive scheme on dimension; reduce the problem of resolution of singularities of $(W,(\mathcal{I}, a), E)$ to that of $(H,(\mathcal{J}, b), D)$, where $H$ is a smooth hypersurface in $W$. The hypersurface $H$ is called a hypersurface of maximal contact, since it contains (contacts) the singular $\operatorname{locus} \operatorname{Sing}(\mathcal{I}, a)$ and since so do its strict transforms throughout any sequence of transformations. The ideal $\mathcal{J}$ on $H$ is usually realized as $\mathcal{J}=\left.C(\mathcal{I})\right|_{H}$, where $C(\mathcal{I})$ is the so-called coefficient ideal of the original ideal $\mathcal{I}$, which is larger than $\mathcal{I}$. (It is worthwhile noting that the mere restriction $\left.\mathcal{I}\right|_{H}$ of the original ideal would fail to provide the inductive scheme in general, and it is necessary to take a larger ideal.) In short, we decrease the dimension by converting the problem on $W$ into the one on the hypersurface of maximal contact $H$ with $\operatorname{dim} H=\operatorname{dim} W-1$.

\subsubsection{Algorithm: modifications and construction of the strand of}

invariants. The above description of the inductive scheme is, however, oversimplified. For an arbitrary triplet $(W,(\mathcal{I}, a), E)$, a hypersurface of maximal contact may not exist at all. In order to guarantee that a hypersurface of maximal contact $H$ exists, we have to take the "companion modification" associated to the weak-order " $w$ ". Furthermore, in order to guarantee that $H$ is transversal to $E$ and hence that we can take $D=\left.E\right|_{H}$ as a simple normal crossing divisor on $H$, we have to take the "boundary modification" associ- 
ated to the invariant " $s$ ". In other words, only after considering the pair of invariants $(w, s)$ and taking the corresponding companion modification and its boundary modification, we can find the triplet $(H,(\mathcal{J}, b), D)$ of dimension one less as described in 0.2.1.2., whose resolution of singularities corresponds to the decrease of the pair of invariants $(w, s)$. (In general, even after modifications, a hypersurface of maximal contact exists only locally, and so does $(H,(\mathcal{J}, b), D)$. Therefore, it is an issue how to globalize this procedure, the important issue which we ignore in this crash course for simplicity.)

Therefore, the actual algorithm realizing the inductive scheme is carried out in such a way that we construct the strand of invariants

$$
i n v_{\text {classical }}=(w, s)(w, s)(w, s) \cdots
$$

by repeating the operations of taking the companion modification, boundary modification, and taking the restriction to a hypersurface of maximal contact, and that at the end we reach the stage where the maximum locus of the strand $i n v_{\text {classical }}$ of invariants coincides with the last hypersurface of maximal contact, which is hence nonsingular and which we choose as the center of blowup. (We remark that, to be precise, at the end we may also reach the stage where the ideal is "monomial", in which case the nonsingular center of blowup can be chosen easily by a combinatorial method.) After the blowup, we repeat the same process. We can repeat the process only finitely many times, since after each blowup the value of the strand of invariants strictly drops and since the set of its values satisfies the descending chain condition, leading to the termination of the algorithm. (See, e.g.,[Vil89] [Vil92] [BM97] [EV00] [EH02] [BV03] [Wło05] [Kol05] [BM07] [Mk07] for details of the construction of the strand of invariants and the corresponding modifications in the classical setting.)

0.2.2. Trouble in positive characteristic. In positive characteristic, however, the examples by R. Narasimhan [Nar83a] [Nar83b] and others [Hau98] [Mk07] demonstrate that there is no hope of finding a hypersurface of maximal contact in general (even after companion or boundary modification), as long as we require it to contain the singular locus and to be nonsingular. This lack of a hypersurface of maximal contact and hence of an apparent inductive scheme is the main source of troubles, which allowed the problem in positive characteristic to elude any systematic attempt to find an algorithm for its solution so far.

0.2.3. Our program: a new approach in the framework of the idealistic filtration. Our program offers a new approach to overcome the 
main source of troubles in the language of the idealistic filtration, which is a refined extension of such classical notions as the idealistic exponent by Hironaka, the presentation by Bierstone-Milman, the basic object by Villamayor, and the marked ideal by Włodarczyk. We devote Part I of the series of papers to introducing the notion of an idealistic filtration, and to establishing its fundamental properties.

0.2.3.1. What is an idealistic filtration? In the classical setting, we consider the pair $(\mathcal{I}, a)$ consisting of an ideal $\mathcal{I} \subset \mathcal{O}_{W}$ on a nonsingular variety $W$ and the aimed multiplicity $a \in \mathbb{Z}_{>0}$. Stalkwise at a point $P \in W$, this is equivalent to considering the collection of pairs $\left\{(f, a) ; f \in \mathcal{I}_{P}\right\}$.

Suppose we interpret the pair $(f, a)$ as a statement saying that "the multiplicity of $f$ is at least $a$ ". In this interpretation, the problem of resolution of singularities (cf. 0.2.1.1.) is, after a sequence of blowups and through transformations and at every point of the ambient space, to negate at least one statement in the collection.

Observe in this interpretation that the following conditions naturally hold:

$$
\left\{\begin{aligned}
& \text { (o) }(f, 0) \quad \forall f \in \mathcal{O}_{W, P},(0, a) \quad \forall a \in \mathbb{Z} \\
& \text { (i) }(f, a),(g, a) \Longrightarrow(f+g, a) \\
& r \in \mathcal{O}_{W, P},(f, a) \Longrightarrow(r f, a) \\
& \text { (ii) }(f, a),(h, b) \Longrightarrow(f h, a+b) \\
& \text { (iii) }(f, a), b \leq a \Longrightarrow(f, b) .
\end{aligned}\right.
$$

Observe also that the problem of resolution of singularities stays unchanged, even if we add the statements derived from the given collection using the above conditions (implications). For example, starting from the given collection $\left\{(f, a) ; f \in \mathcal{I}_{P}\right\}$, the problem stays unchanged even if we consider the new collection $\left\{(f, n) ; f \in \mathcal{I}_{P}^{\lceil n / a\rceil}, n \in \mathbb{Z}_{\geq 0}\right\}$. Our philosophy is that it should be theoretically more desirable to consider the larger or largest collection of statements toward the problem of resolution of singularities.

Accordingly we define an idealistic filtration, at a point $P \in W$, to be a subset $\mathbb{I} \subset \mathcal{O}_{W, P} \times \mathbb{R}$ satisfying the following conditions: 


$$
\left\{\begin{aligned}
& \text { (o) }(f, 0) \in \mathbb{I} \forall f \in \mathcal{O}_{W, P},(0, a) \in \mathbb{I} \forall a \in \mathbb{R} \\
& \text { (i) }(f, a),(g, a) \in \mathbb{I} \Longrightarrow(f+g, a) \in \mathbb{I} \\
& r \in \mathcal{O}_{W, P},(f, a) \in \mathbb{I} \Longrightarrow(r f, a) \in \mathbb{I} \\
& \text { (ii) }(f, a),(h, b) \in \mathbb{I} \quad \Longrightarrow(f h, a+b) \in \mathbb{I} \\
& \text { (iii) }(f, a) \in \mathbb{I}, b \leq a \quad \Longrightarrow(f, b) \in \mathbb{I} .
\end{aligned}\right.
$$

Note that, as a consequence of conditions (o) and (iii), we have

$$
(f, a) \in \mathbb{I} \quad \text { for any } f \in \mathcal{O}_{W, P}, a \in \mathbb{R}_{\leq 0} .
$$

We say an element $(f, a) \in \mathbb{I}$ is at level $a$. Note that we let the level vary in $\mathbb{R}$. Starting from the level varying in $\mathbb{Z}$, we are naturally led to the situation where we let the level varying in the fractions $\mathbb{Q}$ when we start considering the condition (cf. $\mathfrak{R}$-saturation)

$$
\text { (radical) }\left(f^{n}, n a\right) \in \mathbb{I}, n \in \mathbb{Z}_{>0} \Longrightarrow(f, a) \in \mathbb{I},
$$

and then to the situation where we let the level varying in $\mathbb{R}$ when we start considering the condition of continuity

(continuity) $\left(f, a_{l}\right) \in \mathbb{I}$ for a sequence $\left\{a_{l}\right\}$ with $\lim _{l \rightarrow \infty} a_{l}=a \Longrightarrow(f, a) \in \mathbb{I}$.

Note that there is one more natural condition to consider related to the differential operators

(differential) $(f, a) \in \mathbb{I}, d$ a differential operator of degree $t \Longrightarrow(d(f), a-t) \in \mathbb{I}$.

We remark that we do not include condition (radical), (continuity) or (differential) in the definition of an idealistic filtration, even though these conditions play crucial roles when we consider the radical and differential saturations of an idealistic filtration (cf. 0.2.3.2.3.). We also introduce the notion of an idealistic filtration of r.f.g. type (cf. $\S 0.8)$.

We also remark that, given an ideal $\mathcal{I}_{P}$, considering the collection $\{(f, n a)$; $\left.f \in \mathcal{I}_{P}^{n}, n \in \mathbb{Z}_{\geq 0}\right\}$ with additive and multiplicative conditions (i) and (ii) as above is equivalent to considering the Rees algebra $\oplus_{n \in \mathbb{Z}_{\geq 0}} \mathcal{I}_{P}^{n}$. Therefore, the notion of an idealistic filtration can be regarded as a generalization of the notion of the Rees algebra, where the grading takes only nonnegative integers for the latter and the level takes rational or even real values for the former. The properties of the Rees algebra within the context of the problem of resolution of singularities, in connection with the differential operators and integral closure, have also been extensively studied by the recent series of papers by Villamayor 
[Vil06a] [Vil06b] [EV07]. It seems, however, that the consideration of the rational (and real) levels is unique to our approach. We would like to emphasize that the extension of the levels leads to a real difference in carrying out the steps of our algorithm and that it is not a matter of theoretical convenience (cf. Remark 3.2.2.2 (6)).

0.2.3.2. Distinguished features. Being framed in a refinement of the classical notions, our program in the language of the idealistic filtration shares some common spirit with the existing approaches. However, the following four features distinguish our program from them in a decisive way:

\subsection{Leading generator system as a collective substitute for} a hypersurface of maximal contact. Given an idealistic filtration $\mathbb{I} \subset$ $\mathcal{O}_{W, P} \times \mathbb{R}$ at a point $P \in W$, we look at the graded ring of its leading terms $L(\mathbb{I}):=\bigoplus_{n \in \mathbb{Z}_{\geq 0}} L(\mathbb{I})_{n}$ where $L(\mathbb{I})_{n}=\left\{f \bmod \mathfrak{m}_{W, P}^{n+1} ;(f, n) \in \mathbb{I}, f \in \mathfrak{m}_{W, P}^{n}\right\}$. If we fix a regular system of parameters $\left(x_{1}, \ldots, x_{d}\right)$ at $P$ and if we fix a natural isomorphism of $G=\bigoplus_{n \in \mathbb{Z}_{\geq 0}} \mathfrak{m}_{W, P}^{n} / \mathfrak{m}_{W, P}^{n+1}$ with the polynomial ring $k\left[x_{1}, \ldots, x_{d}\right]$, the graded ring $L(\mathbb{I})$ can be considered as a graded $k$-subalgebra of $G=k\left[x_{1}, \ldots, x_{d}\right]$.

Now the fundamental observation is that (if the idealistic filtration is differentially saturated (cf. $\mathfrak{D}$-saturation in 0.2 .3 .2 .3 .) ) for a suitably chosen regular system of parameters, we can choose the generators of $L(\mathbb{I})$, as a graded $k$-subalgebra of $k\left[x_{1}, \ldots, x_{d}\right]$, to be of the form

$$
\left\{x_{i}^{p^{e_{i}}} ; e_{i} \in \mathbb{Z}_{\geq 0}\right\}_{i \in I} \quad \text { for some } \quad I \subset\{1, \ldots, d\}
$$

when we are in positive characteristic $\operatorname{char}(k)=p>0$. We define a leading generator system of the idealistic filtration to be a set of elements $\left\{\left(h_{i}, p^{e_{i}}\right)\right\}_{i \in I} \subset \mathbb{I}$ whose leading terms give rise to the set of generators as above, i.e., $h_{i} \bmod$ $\mathfrak{m}_{W, P}^{p^{e_{i}+1}}=x_{i}^{p_{i}}$ for $i \in I$. We emphasize that the leading terms of the elements in the leading generator system lie in degrees $p^{0}, p^{1}, p^{2}, p^{3}, \ldots$, and hence that the leading generator system may not form (a part of) a regular system of parameters when we are in positive characteristic $\operatorname{char}(k)=p>0$. In the example by R. Narasimhan [Nar83a] [Nar83b], where there is no nonsingular hypersurface of maximal contact, there is no leading term of degree one in any leading generator system. When we are in characteristic zero $\operatorname{char}(k)=0$, in contrast, we can choose the generators of $L(\mathbb{I})$ to be concentrated all in degree one, i.e., of the form

$$
\left\{x_{i}\right\}_{i \in I} \quad \text { for some } \quad I \subset\{1, \ldots, d\} .
$$


Accordingly, we can take a leading generator system to be a set of elements $\left\{\left(h_{i}, 1\right)\right\}_{i \in I} \subset \mathbb{I}$ with $h_{i} \bmod \mathfrak{m}_{W, P}^{2}=x_{i}$ for $i \in I$. If we look at the classical algorithm(s), then a hypersurface of maximal contact (locally at $P$ ) is given by $\left\{h_{i}=0\right\}$ (for some $i \in I$ ). Since the leading term of $h_{i}$ is linear, it is guaranteed to define a nonsingular hypersurface.

Although forming a clear contrast, the case in positive characteristic and the case in characteristic zero should not be considered as two separate entities. Rather, the case in characteristic zero should be considered as a special case of the uniform phenomenon: Traditionally we define the characteristic char $(k)$ to be the (non-negative) generator of the set of the annihilators in $\mathbb{Z}$ of the unit "1" in the field $k$. However, as Hironaka points out in [Hir70], for the purpose of considering the problem of resolution of singularities, it is more natural to adopt the following definition

$$
p=\inf \left\{n \in \mathbb{Z}_{>0} ; n \cdot 1=0 \in k\right\} .
$$

Therefore, the case of characteristic being zero in the traditional sense corresponds to the case of $p=\infty$ in this convention. In other words, we expect the behavior in characteristic zero to be similar to the one in positive characteristic with large $p$, and ultimately to lie at the limit when $p \rightarrow \infty$. Accordingly, in characteristic zero with $p=\infty$, the (virtual) leading terms of the leading generator system in degrees $p^{1}=p^{2}=\cdots=\infty$ are invisible (non-existent), while the actual leading terms are concentrated all in degree $\lim _{p \rightarrow \infty} p^{0}=1$.

That is to say, we consider the notion of a hypersurface of maximal contact in characteristic zero to be a special case of the notion of a leading generator system, which is valid in all characteristics. Accordingly, we use the notion of a leading generator system as a collective substitute in positive characteristic for the notion of a hypersurface of maximal contact in characteristic zero in the process of constructing an algorithm according to our program.

We would like to remark that, for the purpose of studying a singularity, the idea of analyzing the leading terms of its defining ideal is nothing new, and so classical as is the term "tangent cone". Even in a more specific subject of the problem of resolution of singularities and in the context of studying the Rees algebra, Hironaka, Oda, and Giraud, among others, realized its importance early on in relation to the effect of taking the differential saturation and/or to the notion of a standard basis (cf. [Hir70] [Oda73] [Oda83] [Oda87] [Gir75]). The fundamental observation mentioned above appears in [Oda87]. In fact, the recent approaches (cf. 0.5.2), e.g., the one by Villamayor via "generic projection" [Vil06a] [Vil06b] [EV07], referring to Hironaka's $\tau$-invariant, the one by Włodarczyk via the notion of " $p$-order" [Wło07], referring to [Gir75] as its 
inspirational source, and the one by Hironaka himself [Hir05] [Hir06], all find renewed interests in this classical idea combined with novel developments of their own. Our approach via the notion of the leading generator system is no exception.

0.2.3.2.2. Enlargement vs. restriction. (Construction of the strand of invariants through enlargements (modifications) of an idealistic filtration, and without using restriction to a hypersurface of maximal contact.) At first sight, the introduction of the notion of a leading generator system does not seem to contribute toward overcoming the main source of troubles at all. Recall (cf. 0.2.1.3.) that in the classical setting in characteristic zero the strand of invariants is constructed in such a way that a unit $(w, s)$ is added to the strand constructed so far every time we decrease the dimension by one, and then continue the construction by restricting ourselves to a hypersurface of maximal contact. Nonsingularity of a hypersurface of maximal contact is absolutely crucial in order to continue the construction by restriction. Therefore, in the new setting in positive characteristic where we use a leading generator system, we seem to fail to construct the strand of invariants if any of the elements in the leading generator system defines a singular hypersurface. However, in the construction of the strand of invariants in the new setting, we do not use any restriction but only use enlargements (modifications) of the idealistic filtration. In fact, starting from a given idealistic filtration on a nonsingular variety $W$, we construct the triplet of invariants $(\sigma, \widetilde{\mu}, s)$, where $\sigma$ reflects the degrees of the leading terms of a leading generator system, and $\widetilde{\mu}$ and $s$ are the weak-order (with respect to a leading generator system) and the invariant determined by the boundary, respectively, corresponding to the invariants $w$ and $s$ as before. In the classical setting, after taking the corresponding companion modification and boundary modification, we take a hypersurface of maximal contact at this point and continue the process by taking the restriction to it. In the new setting, however, after taking the companion modification and boundary modification, we consider a leading generator system of the newly modified idealistic filtration and continue the process. In other words, in the new setting, we construct the strand of invariants in the following form

$$
i n v_{\text {new }}=(\sigma, \widetilde{\mu}, s)(\sigma, \widetilde{\mu}, s)(\sigma, \widetilde{\mu}, s) \cdots,
$$

and the construction is done only through enlargement keeping the ambient space $W$ intact, and hence the crucial nonsingularity intact.

It is worthwhile noting that $\widetilde{\mu}$ is independent of the choice of a leading 
generator system, which is a priori needed for its definition, and hence is an invariant canonically attached to the idealistic filtration (if it is appropriately saturated (See 0.2.3.2.3. below.)). This implies that the strand of invariants $i n v_{\text {new }}$ is also canonically determined globally. Therefore, we see that the center of each blowup in our algorithm, which is the maximum locus of the strand of invariants, is also canonically and globally defined, without the so-called Hironaka's trick needed in the classical setting (cf. 0.2.3.2.3. and [Wło05]).

In Part II, we will define the two basic invariants denoted by $\sigma$ and $\widetilde{\mu}$ in the context of an idealistic filtration as above. They form the building blocks for constructing the strand of invariants (together with invariant $s$ related to the boundary). Some of their properties which are straightforward in characteristic zero, e.g., the upper semi-continuity, become highly non-trivial in positive characteristic and are also discussed in Part II.

Discussion of the modifications is one of the main themes of Part III, where the classical notion of the companion modification and that of the boundary modification find their perfect analogs in the context of the enlargements of an idealistic filtration with respect to a leading generator system.

0.2.3.2.3. Saturations. It is important in our program to make a given idealistic filtration "larger" without changing the associated problem of resolution of singularities. Ultimately, we would like to find the largest of all such (with respect to a certain fixed kind of operations " $X$ "), leading to the notion of the $(X$-)saturation. Dealing with the saturated idealistic filtration, we expect to extract more intrinsic information toward a solution of the problem of resolution of singularities (e.g. invariants which are independent of the choice of a leading generator system in the new setting, or the choice of a hypersurface of maximal contact in the classical setting). The two key saturations in our program are the differential saturation (called the $\mathfrak{D}$-saturation for short, with respect to the operation of taking differentiations) and the radical saturation (called the $\mathfrak{R}$-saturation for short, with respect to the operation of taking the $n$-th roots (radicals)), the latter being equivalent to taking the integral closure (for an idealistic filtration of r.f.g. type). (The operation of taking the coefficient ideal and the operation of taking the "homogenization" in the sense of [Wło05] share the same spirit with $\mathfrak{D}$-saturation. In fact, we can obtain new formulas for the coefficient ideal and the homogenization as byproducts of the notion of the $\mathfrak{D}$-saturation of an idealistic filtration. See [Mk07] for details. We also invite the reader to look at [Kol05], which discusses several extensions of the idea of homogenization.) At the center of our program sits the analysis of the 
interaction of these two saturations (See also [Vil06a] [Vil06b] [EV07] for the related results in the study of the Rees algebra.), leading to the notion of the bi-saturation (called the $\mathfrak{B}$-saturation) and its explicit description as the $\mathfrak{R D}$ saturation. Note that the notion of a leading generator system in 0.2.3.2.1. is defined only through $\mathfrak{D}$-saturation, and the new nonsingularity principle in 0.2.3.2.4. only through $\mathfrak{B}$-saturation.

0.2.3.2.4. New nonsingularity principle. There is another problem which comes along with using a leading generator system as a collective substitute for a hypersurface of maximal contact. In the classical setting in characteristic zero, what guarantees the nonsingularity of the center is the nonsingularity of a hypersurface of maximal contact (cf. 0.2.1.3.). In our new setting in positive characteristic, we no longer have this guarantee. In fact, at the intermediate stage of the construction of the strand of invariants, the leading generator system may not be (a part of) a regular system of parameters and hence may define a singular subscheme. We observe, however, that at the end of the construction of the strand of invariants the enlarged idealistic filtration takes such a special form that guarantees the corresponding leading generator system to be (a part of) a regular system of parameters. The maximum locus of the strand of the invariants, which we choose as the center, is defined by this leading generator system, and hence is nonsingular. We call this observation the new nonsingularity principle of the center.

We would like to remark that, as the new nonsingularity principle is indispensable in our program, the use of $\mathfrak{B}$-saturation (and hence of $\mathfrak{R}$-saturation) is essential in executing our algorithm. This feature distinguishes our program not only from the existing and classical methods but also from the other proposed approaches (cf. 0.5.2), where the conceptual importance of $\mathfrak{R}$-saturation (taking the integral closure) is emphasized in defining some equivalence classes but never used explicitly in executing their algorithms.

0.2.3.3. Uniformity of our program in all characteristics. It should be emphasized that our program is not designed to come up with an esoteric strategy peculiar to the situation in positive characteristic, but rather intended to develop a uniform point of view toward the problem of resolution of singularities valid in all characteristics. Part IV is devoted to letting this point of view manifest itself in the form of an algorithm, summarizing all the ingredients of the program. 


\section{§0.3. Algorithm constructed according to the program}

0.3.1. Algorithm in characteristic zero. Aiming at uniformity, our program makes perfect sense and works just as well in characteristic zero, leading to a new algorithm slightly different from the existing ones. We will demonstrate in Part IV how the distinguished features of our program described in 0.2.3.2. work in the new algorithm.

0.3.2. Algorithm in positive characteristic; the remaining problem of termination. The algorithm in characteristic zero, now through uniformity, serves as a prototype toward establishing an algorithm in positive characteristic. In fact, we can carry out almost all the procedures of our algorithm in positive characteristic, forming a perfect parallel to the case in characteristic zero, except for the problem of termination.

0.3.2.1. Termination. It is easy to see that in characteristic zero the invariants constituting the strand, constructed according to the program, have bounded denominators, and hence that the strand takes its value in the set satisfying the descending chain condition. Since the value of the strand strictly drops after each blowup, we conclude that the algorithm terminates after finitely many steps. However, in positive characteristic, we can not exclude the possibility that the denominators may increase indefinitely as we carry out the processes (blowups) of the algorithm. (In the unit $(\sigma, \widetilde{\mu}, s)$ for the strand, the values of invariant $\sigma$ and $s$ are easily seen to satisfy the descending chain condition. Therefore, more specifically, the only issue is the boundedness of the denominators for the values of $\widetilde{\mu}$, which are fractional.) Therefore, we do not know at the moment if the algorithm terminates after finitely many steps.

The problem of termination remains as the only missing piece in our quest of establishing an algorithm for resolution of singularities in positive characteristic according to the program. The details will be discussed in Part IV.

\section{§0.4. Assumption on the base field}

We carry out our entire program assuming that the base field $k$ is algebraically closed field of characteristic $\operatorname{char}(k)=p \geq 0$.

Our definition of a leading generator system, the key notion of the program, at a closed point $P \in W$ where $W$ is a variety of dimension $d$ smooth over $k$, needs the assumption of the base field being algebraically closed, since we use the fact $\mathcal{O}_{W, P} / \mathfrak{m}_{W, P} \cong k$ and the natural isomorphism $G=\bigoplus_{n \geq 0} \mathfrak{m}_{W, P}^{n+1} / \mathfrak{m}_{W, P}^{n}$ 
$\cong k\left[x_{1}, \ldots, x_{d}\right]$ with respect to a fixed regular system of parameters $\left(x_{1}, \ldots, x_{d}\right)$, as well as the fact that we can take the $p$-th root of any element within $k$ (when $\operatorname{char}(k)=p>0$ ). We briefly mention below what happens if we loosen the assumption on the base field.

0.4.1. Perfect case. Suppose that the base field $k$ is perfect, but not necessarily algebraically closed. Upon completion, the algorithm constructed according to the program should be equivariant under any group action (cf. Part IV). Therefore, as long as the base field $k$ is perfect, we see that the algorithm established over its algebraic closure $\bar{k}$ descends to the one over the original base field $k$, utilizing the equivariance under the action of the Galois $\operatorname{group} \operatorname{Gal}(\bar{k} / k)$.

0.4.2. Non-perfect case. Over a non-perfect field $k$, we even have to start distinguishing the notion of being regular and that of being smooth over $k$. The discussions, including the one on how we may try to reduce the nonperfect case to the perfect case using the Lefschetz Principle type argument, will be given in Part IV.

\section{$\S 0.5$. Other methods and approaches}

0.5.1. Brief history. First we briefly mention the history of a few of the other methods and approaches than the algorithmic approach we follow toward the problem of resolution of singularities in positive characteristic. We refer the reader to [Lip75] [Moh96] [HLOQ00] for a more detailed account.

Resolution of singularities for curves is a classical result, with many of its ideas and methods leading to the higher dimensional cases even to this day.

Among several results for surfaces, the most general one seems to be given by [Lip69] [Lip78], which establish resolution of singularities of an arbitrary excellent scheme in dimension 2.

It is [Zar40] that initiated the strategy to establish local uniformizations first, with the theory of valuations as the central tool, and then by patching them to establish resolution of singularities globally. The theory of local uniformization has been further developed by many people [Abh66] [Cos00] [Kuh97] [Kuh00]. We should mention the approaches by [Tei03] [Spi04] toward local uniformization in higher dimensions.

Jung's idea of taking the (generic) projection provides many useful approaches toward the problem of resolution of singularities. [Abh66] uses the method of Albanese projecting from a singular point, combined with the theory 
of local uniformization, to resolve singularities of a threefold $X$ when char $(k)$ is greater than $(\operatorname{dim} X) !=6$. A simplified proof has been recently given by [Cut06], which also discusses the potential and problems if one tries to extend the method to higher dimensions. There are attempts to study the problem in the remaining characteristic $\operatorname{char}(k)=2,3,5$ by [Cos87] [Moh96] [Cos04] [Pil04] in dimension 3.

Without any restriction on the dimension of a variety or on the base field $k$, the most remarkable development in the vicinity of the problem of resolution of singularities is arguably the method of alteration initiated by de Jong [dJ96]. Given a variety $X$, it constructs a proper and generically finite morphism $f: Y \rightarrow X$ from a regular variety $Y$. (In characteristic zero, one can refine the method of alteration to realize $f$ as a birational map. See [AdJ97] [BP96] [Par99] for details.) The structure of $f$ is rather obscure, though its existence follows nicely and simply by regarding $X$ as a family of curves fibered over a variety of dimension one less and hence by paving a way to apply induction. The method of alteration even works in mixed characteristics or with integral schemes over $\mathbb{Z}$, and hence it allows a wide range of applications for arithmetic purposes.

0.5.2. Recent announcements of other new approaches. During the preparation of the first draft for Part I, we were informed that Hironaka announced a program of resolution of singularities in all characteristics $p>0$ and in all dimensions at the summer school in Trieste 2006 (cf. [Hir06]). In the course of revision, we also learned of a program by Villamayor [Vil06a] [Vil06b] [EV07] and one by Włodarczyk [Wło07], each pursuing its own direction different from ours using the method of "generic projection" and the notion of " $p$-order", respectively, toward resolution of singularities in positive characteristic. We have not had the time to analyze these approaches in comparison to ours, while none of them, including ours, seems to claim a complete proof for the moment. We refer the reader to their research papers for the precise contents.

\section{$\S 0.6$. Origin of our program and its name}

This series of papers is a joint work of H. Kawanoue and K. Matsuki as a whole. However, the program forming the backbone of the series was conceived in its entirety by the first author toward his Ph.D. thesis, and revealed to the second author in the summer of 2003 at a private seminar held at Purdue University as a blueprint toward constructing an algorithm for resolution of 
singularities in positive characteristic. As such all the essential ideas are due to the first author. The only contribution of the second author was to help the first author and jointly bring these ideas together converging into a coherent algorithm. Part I, which represents the main portion of the afore-mentioned Ph.D. thesis, bears only the name of the first author.

In the process of writing this series of papers, we felt it is not only convenient but also necessary to give a proper name to our program. After its main framework "the idealistic filtration", we decided to call it the Idealistic Filtration Program, abbreviated as the IFP.

\section{§0.7. Acknowledgement}

Our entire project could only be possible through the guidance and encouragement of Professor Shigefumi Mori both at the personal level and in the mathematical context. He not only shared his insight generously with us, but also on several occasions in the development of the IFP showed us directly some key arguments to bring us forward. Professor Masaki Kashiwara also gave us an invaluable and enthusiastic support, without which the project would have dissipated into the air.

We thank Professors Edward Bierstone, Pierre Milman, Orlando Villamayor, and Herwig Hauser, from whom we learned most on the subject of resolution of singularities, where the tutoring was given in the form of publications and personal correspondences. Only through their teaching, we started understanding the greatest ideas of [Hir64]. Many of the ideas of our project, therefore, find their origins in [Hir64] as well as in the papers of our teachers cited above. Our indebtedness to Professor Heisuke Hironaka, whose influence was decisive for us to enter the subject, is immeasurable.

It is a pleasure to acknowledge the helpful comments and suggestions we received from Professors Donu Arapura, Johan de Jong, Joseph Lipman, TsuongTsieng Moh, Tadao Oda, Bernd Ulrich, Jarosław Włodarczyk.

We are grateful to the referee for many valuable comments and for bringing several of the classical references, which we were not aware of, to our attention.

Special thanks go to Hidehisa Alikawa, Takeshi Nozawa, and Masahiko Yoshinaga, who were both good friends and patient listeners in Room 120 for the graduate students of Research Institute for Mathematical Sciences in Kyoto at the dawn of the IFP. 


\section{$\S 0.8$. Outline of Part I}

Following the itemized table of contents at the beginning, we describe the outline of the structure of Part I below.

At the end of the introduction in Chapter 0, we give a brief description of the preliminaries to read Part I and the subsequent series of papers. In Chapter 1 , we recall some basic facts on the differential operators, especially those in positive characteristic. Both in the description of the preliminaries and in Chapter 1, our purpose is not to exhaustively cover all the material, but only to minimally summarize what is needed to present our program and to fix our notation. For example, an elementary characterization, in terms of the differential operators, of an ideal generated by the $p^{e}$-th power elements in characteristic $p=\operatorname{char}(k)>0$ is included only due to the lack of an appropriate reference. We should emphasize here that the use of the logarithmic differential operators is indispensable in our setting in the language of the idealistic filtration (See Remark 1.2.2.3).

Chapter 2 is devoted to establishing the notion of an idealistic filtration, and its fundamental properties. The most important ingredient of Chapter 2 is the analysis of the $\mathfrak{D}$-saturation and $\mathfrak{R}$-saturation and that of their interaction. In our algorithm, given an idealistic filtration, we always look for its bi-saturation, called the $\mathfrak{B}$-saturation, which is both $\mathfrak{D}$-saturated and $\mathfrak{R}$ saturated and which is minimal among such containing the original idealistic filtration. The existence of the $\mathfrak{B}$-saturation is theoretically clear. However, we do not know a priori whether we can reach the $\mathfrak{B}$-saturation by a repetition of $\mathfrak{D}$-saturations and $\mathfrak{R}$-saturations starting from the given idealistic filtration, even after infinitely many times. The main result here is that the $\mathfrak{B}$-saturation is actually realized if we take the $\mathfrak{D}$-saturation and then $\mathfrak{R}$-saturation of the given one, each just once in this order. In our algorithm, we do not deal with an arbitrary idealistic filtration, but only with those which are generated by finitely many elements with rational levels. We say they are of r.f.g. type (short for "rationally and finitely generated"). It is then a natural and crucial question if the property of being of r.f.g. type is stable under $\mathfrak{D}$-saturation and $\mathfrak{R}$-saturation. We find somewhat unexpectedly that the argument of M. Nagata (cf. [Nag57]), which was originally developed to answer some questions posed by P. Samuel regarding the asymptotic behavior of ideals, is tailor-made to establish the stability under $\mathfrak{R}$-saturation (while the stability under $\mathfrak{D}$-saturation is elementary). Since the use of $\mathfrak{R}$-saturation (together with the use of $\mathfrak{D}$ saturation) and the introduction of the rational levels are essential in executing our algorithm, so is the stability of r.f.g. type. 
In Chapter 3, through the analysis of the leading terms of an idealistic filtration (which is $\mathfrak{D}$-saturated), we define the notion of a leading generator system, which, as discussed in 0.2.3.2.1., plays the role of a collective substitute for the notion of a hypersurface of maximal contact.

Chapter 4 is the culmination of Part I, establishing the new nonsingularity principle of the center for an idealistic filtration which is $\mathfrak{B}$-saturated. Its proof is given via three somewhat technical but important lemmas, which we will use again later in the series of papers.

Our theory in Part I is mainly local, dealing almost exclusively with an idealistic filtration over the local ring of a closed point on a nonsingular ambient variety. The global theory toward constructing an algorithm will be discussed in the subsequent papers.

The main purpose of Part I is to establish the foundation of our program toward constructing an algorithm for resolution of singularities. However, we believe that the results on the idealistic filtration we discuss here in Part I, notably the analysis leading to the explicit description of the $\mathfrak{B}$-saturation, stability of r.f.g. type, and the new nonsingularity principle, are of interest on their own in the subject of the ideal theory in commutative algebra.

This finishes the discussion of the outline of Part I.

\section{$\S 0.9$. Preliminaries}

We summarize a few of the preliminaries in order to read Part I and the subsequent series of papers.

0.9.1. The language of schemes. Our entire argument is carried out in the language of schemes. For example, a variety is an integral separated scheme of finite type over $k$. Accordingly, when we say "points", we refer to the scheme-theoretic points and do not confine ourselves to the closed points, which correspond to the geometric ones in the classical setting. Thus the invariants that we construct will be defined over all the scheme-theoretic points, and not confined to the closed points. However, some of the key notions of our program, notably that of a leading generator system, are only defined at the level of the closed points, and the values of the invariants over the non-closed points are given only indirectly through their upper or lower semi-continuity (cf. Part II).

Our program is not conceived in the language of schemes originally. Rather, it has its origin in the concrete analysis and computation in terms of the coordinates at the closed points. As such, it can be applied to many 
other "spaces" than algebraic varieties over $k$, where the same analysis and computation can be applied to the coordinates at its closed points. The task of presenting a set of axiomatic conditions for the IFP to function, and that of listing explicitly the spaces within its applicability will be dealt with elsewhere.

0.9.2. Basic facts from commutative algebra. For the basic facts in commutative algebra, we try to use [Mat86] as the main source of reference.

0.9.3. Multi-index notation. When we have the multi-variables, either as the indeterminates in the polynomial ring or as a regular system of parameters, we often use the following multi-index notations:

$$
\left\{\begin{array}{c}
X=\left(x_{1}, \ldots, x_{d}\right), \quad I=\left(i_{1}, \ldots, i_{d}\right) \in \mathbb{Z}_{\geq 0}^{d} \\
|I|=\sum_{\alpha=1}^{d} i_{\alpha}, \quad X^{I}=\prod_{\alpha=1}^{d} x_{\alpha}^{i_{\alpha}} \\
\left(\begin{array}{c}
I \\
J
\end{array}\right)=\prod_{\alpha=1}^{d}\left(\begin{array}{c}
i_{\alpha} \\
j_{\alpha}
\end{array}\right) \quad \text { for } \quad J=\left(j_{1}, \ldots, j_{d}\right) \in \mathbb{Z}_{\geq 0}^{d} \\
\text { where }\left(\begin{array}{l}
i \\
j
\end{array}\right)=\frac{i !}{(i-j) ! j !} \in \mathbb{Z}_{\geq 0} \text { denotes the binomial coefficient, } \\
\left(\text { We also use the convention that, whenever } i_{\alpha}<j_{\alpha}, \text { we set }\left(\begin{array}{c}
i_{\alpha} \\
j_{\alpha}
\end{array}\right)=0 .\right) \\
\partial_{X^{J}}=\frac{\partial^{|J|}}{\partial_{x_{1}}^{i_{1}} \cdots \partial_{x_{d}}^{i_{d}}} \quad \text { (expressed by } \partial_{J} \text { for short) } \\
\mathbf{e}_{\alpha}=(0, \ldots, 1, \ldots, 0) .
\end{array}\right.
$$

\section{Chapter 1. Basics on Differential Operators}

The purpose of this chapter is to give a brief account of the differential operators, which play a key role in the Idealistic Filtration Program.

We would like to mention that it is through reading the papers [Hir70] [Oda73] that our attention was first brought to the importance of the higher order differential operators in the context of the problem of resolution of singularities in positive characteristic.

Our main reference is EGA IV $\S 16$ [Gro67], where all that we need, especially the properties of the higher order differential operators of Hasse-Schmidt type in positive characteristic, and much more, is beautifully presented. We only try to extract some basic facts and discuss them in the form that suits our limited purposes. 


\section{$\S 1.1 . \quad$ Definitions and first properties}

1.1.1. Definitions. Recall that the base field $k$ is assumed to be an algebraically closed field of $\operatorname{char}(k) \geq 0$.

Definition 1.1.1.1. Let $R$ be a $k$-algebra. We use the following notation:

$$
\begin{aligned}
\mu: R \otimes_{k} R & \rightarrow R \quad \text { the multiplication map, } \quad I:=\operatorname{ker}(\mu) \quad \text { the kernel of } \mu, \\
P_{R}^{n} & =R \otimes_{k} R / I^{n+1}, \quad q_{n}: R \rightarrow R \otimes_{k} R \rightarrow P_{R}^{n} \quad \text { for } n \in \mathbb{Z}_{\geq 0}
\end{aligned}
$$

where $q_{n}$ is the composition of the map to the second factor with the projection, i.e.,

$$
q_{n}(r)=\left(1 \otimes r \bmod I^{n+1}\right) \quad \text { for } \quad r \in R .
$$

A differential operator $d$ of degree $\leq n$ on $R$ (over $k$ ) for $n \in \mathbb{Z}_{\geq 0}$ is a map $d: R \rightarrow R$ of the form

$$
d=u \circ q_{n} \quad \text { with } \quad u \in \operatorname{Hom}_{R}\left(P_{R}^{n}, R\right)
$$

(We note that the $R$-module structure on $P_{R}^{n}$ is inherited from the $R$-module structure on $R \otimes_{k} R$ given by the multiplication on the first factor.)

We denote the set of differential operators of degree $\leq n$ on $R$ by $\operatorname{Diff}_{R}^{n}$, i.e.,

$$
\operatorname{Diff}_{R}^{n}:=\left\{d=u \circ q_{n} ; u \in \operatorname{Hom}_{R}\left(P_{R}^{n}, R\right)\right\} .
$$

(Note that Diff $R_{R}^{n}$ inherits the $R$-module structure from the one on $\operatorname{Hom}_{R}$ $\times\left(P_{R}^{n}, R\right)$.)

We call $\operatorname{Diff}_{R}=\bigcup_{n=0}^{\infty} \operatorname{Diff}_{R}^{n}$ (cf. Lemma 1.1.2.1) the set of the differential operators on $R$ (over $k$ ).

For a subset $T \subset R$, we also use the following notation

$$
\operatorname{Diff}_{R}^{n}(T)=\left(\left\{d(r) ; d \in \operatorname{Diff}_{R}^{n}, r \in T\right\}\right) .
$$

\subsubsection{First properties.}

Lemma 1.1.2.1. $\quad$ Let the situation and notation be the same as in Definition 1.1.1.1.

(1) Let $d$ be a k-linear map $d: R \rightarrow R$. Then $d$ is a differential operator of degree $\leq n$, i.e., $d \in \operatorname{Diff}_{R}^{n}$ if and only if $d$ satisfies the Leibnitz rule of degree 
$n$ :

$$
\sum_{T \subset S_{n+1}}(-1)^{|T|}\left(\prod_{s \in S_{n+1} \backslash T} r_{s}\right) d\left(\prod_{s \in T} r_{s}\right)=0
$$

where $S_{n+1}=\{1,2, \ldots, n, n+1\}$ and $r_{s} \in R$ for $s \in S_{n+1}$.

(2) The natural map

$$
\phi_{R}: \operatorname{Hom}_{R}\left(P_{R}^{n}, R\right) \rightarrow \operatorname{Diff}_{R}^{n},
$$

given by $d=\phi_{R}(u)=u \circ q_{n}$ for $u \in \operatorname{Hom}_{R}\left(P_{R}^{n}, R\right.$ ), is bijective (and actually an isomorphism between $R$-modules).

(3) If $R$ is finitely generated as an algebra over $k$, then $P_{R}^{n}$ is finitely generated as an $R$-module, and so is $\operatorname{Hom}_{R}\left(P_{R}^{n}, R\right) \stackrel{\sim}{\rightarrow} \operatorname{Diff}_{R}^{n}$.

(4) Let $R^{\prime}$ be the localization $R_{S}$ of $R$ with respect to a multiplicative set $S \subset R$ or the completion $\widehat{R}$ of $R$ with respect to a maximal ideal $\mathfrak{m} \subset R$. We define the $\operatorname{map} \operatorname{Diff}_{R}^{n} \rightarrow \operatorname{Diff}_{R^{\prime}}^{n}$ so that the following diagram commutes

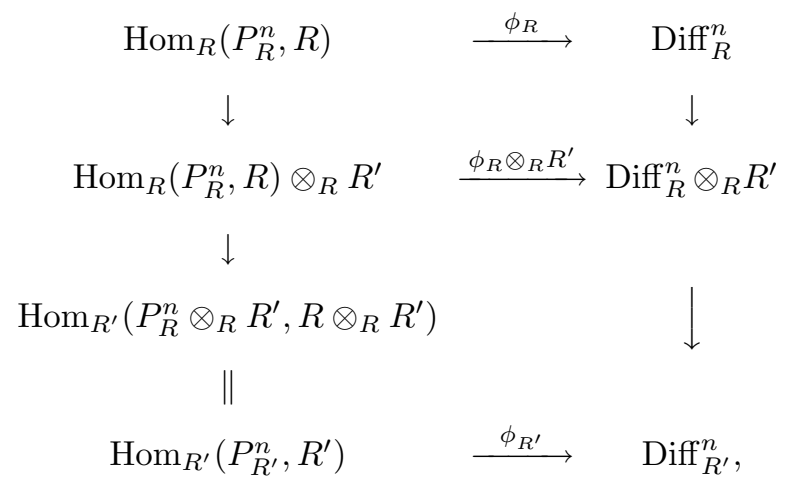

where the vertical arrows are the natural maps.

Consequently, the bijections are compatible with localization and completion.

Moreover, if $R$ is essentially of finite type over $k$, then the second vertical arrow on the left is an isomorphism, and hence so is the second vertical arrow on the right.

(5) Let $d \in \operatorname{Diff}_{R}^{n}$ be a differential operator of degree $\leq n$ on $R$. Then $d$ is a differential operator of degree $\leq m$ for any $n \leq m$. That is to say,

$$
\operatorname{Diff}_{R}^{n} \subset \operatorname{Diff}_{R}^{m} \quad \text { for } n \leq m .
$$

With respect to these inclusions, $\left\{\operatorname{Diff}_{R}^{n}\right\}_{n \in \mathbb{Z}_{\geq 0}}$ forms a projective system. 
(6) Let $d \in \operatorname{Diff}_{R}^{n}$ be a differential operator of degree $\leq n$ on $R$, and $d^{\prime} \in \operatorname{Diff}_{R}^{n^{\prime}}$ be a differential operator of degree $\leq n^{\prime}$ on $R$. Then the composition $d \circ d^{\prime}$ is a differential operator of degree $\leq n+n^{\prime}$ on $R$, i.e., $d \circ d^{\prime} \in \operatorname{Diff}_{R}^{n+n^{\prime}}$.

(7) Let $R$ be an algebra essentially of finite type over $k, I \subset R$ an ideal, and let $R^{\prime}$ be as in (4). Then we have

$$
\operatorname{Diff}_{R}^{n}(I) R^{\prime}=\operatorname{Diff}_{R^{\prime}}^{n}\left(I R^{\prime}\right)
$$

Proof.

(1) We refer the reader to Proposition (16.8.8) in EGA IV $\S 16$ [Gro67] for a proof.

(2) The isomorphism $\phi_{R}$ is the one mentioned in (16.8.3.1) in EGA IV $\S 16$ [Gro67].

(3) Suppose $R$ is finitely generated as an algebra over $k$. Let $X=\left\{x_{1}, \ldots, x_{t}\right\}$ be a set of generators for $R$ over $k$. We see that $P_{R}^{n}$ is generated by $\left\{q_{n}\left(X^{I}\right) ; I \in \mathbb{Z}_{\geq 0}^{t}\right\}$ as an $R$-module (cf. the first note in Definition 1.1.1.1). We also see, by the relation $\prod_{s \in S_{n+1}}\left(1 \otimes r_{s}-r_{s} \otimes 1\right)=0$ in $P_{R}^{n}$, that $q_{n}\left(X^{I}\right)$ for any $I \in \mathbb{Z}_{\geq 0}^{t}$ belongs to the $R$-span of $\left\{q_{n}\left(X^{I}\right) ; I \in \mathbb{Z}_{\geq 0}^{t},|I| \leq n\right\}$. Therefore, we conclude that $P_{R}^{n}$ is finitely generated as an $R$-module and hence that so is $\operatorname{Hom}_{R}\left(P_{R}^{n}, R\right) \stackrel{\sim}{\rightarrow} \operatorname{Diff}_{R}^{n}$.

(4) Compatibility of the bijections with localization and completion follows immediately from the definitions and from the fact that $P_{R}^{n} \otimes_{R} R^{\prime}=P_{R^{\prime}}^{n}$. In order to verify the "Moreover" part, it suffices to show the assertion assuming that $R$ is finitely generated as an algebra over $k$. Then since the extension $R \rightarrow R^{\prime}$ is flat and since $P_{R}^{n}$ is finitely generated as an $R$-module by $(3)$, the second vertical arrow on the left is an isomorphism, and hence so is the second vertical arrow on the right.

(5) The natural surjection $P_{R}^{m}=\left(R \otimes_{k} R\right) / I^{m+1} \rightarrow P_{R}^{n}=\left(R \otimes_{k} R\right) / I^{n+1}$ for $n \leq m$ induces the injection $\operatorname{Hom}_{R}\left(P_{R}^{n}, R\right) \hookrightarrow \operatorname{Hom}_{R}\left(P_{R}^{n+1}, R\right)$ and hence the inclusion $\operatorname{Diff}_{R}^{n} \subset \operatorname{Diff}_{R}^{m}$. It is clear that $\left\{\operatorname{Diff}_{R}^{n}\right\}_{n \in \mathbb{Z}_{\geq 0}}$ forms a projective system with respect to these inclusions.

(6) We refer the reader to Proposition (16.8.9) in EGA IV $\S 16$ [Gro67].

(7) When $R^{\prime}=\widehat{R}$, the equality $\operatorname{Diff}_{R}^{n}(I) R^{\prime}=\operatorname{Diff}_{R^{\prime}}^{n}\left(I R^{\prime}\right)$ follows from the "Moreover" part of (4) and from the fact that the differential operators are continuous with respect the $\mathfrak{m}$-adic topology (the latter being a consequence of the Leibnitz rule).

Thus we give a proof of the equality only when $R^{\prime}=R_{S}$ in the following. Since the inclusion $\operatorname{Diff}_{R}^{n}(I) R_{S} \subset \operatorname{Diff}_{R_{S}}^{n}\left(I R_{S}\right)$ follows easily from the 
"Moreover" part of (4), we have only to show the opposite inclusion

$$
\operatorname{Diff}_{R}^{n}(I) R_{S} \supset \operatorname{Diff}_{R_{S}}^{n}\left(I R_{S}\right) .
$$

Take $f=s^{-1} r \in I R_{S}$ with $r \in R, s \in S$, and take $d \in \operatorname{Diff}_{R_{S}}^{n}$. We want to show $d(f) \in \operatorname{Diff}_{R}^{n}(I) R_{S}$. Set $r_{1}=\cdots=r_{n}=s, r_{n+1}=f$. Applying the Leibnitz rule of degree $n$ for $d \in \operatorname{Diff}_{R_{S}}^{n}$, we have

$$
-s^{n} d(f)+\sum_{\{n+1\} \varsubsetneqq T}(-1)^{|T|} s^{n+1-|T|} d\left(s^{|T|-2} r\right)+\sum_{n+1 \notin T}(-1)^{|T|} f s^{n-|T|} d\left(s^{|T|}\right)=0
$$

where the first term in the left hand side corresponds to the range $T=\{n+1\}$. Since $d \in \operatorname{Diff}_{R_{S}}^{n}=\operatorname{Diff}_{R}^{n} R_{S}$ by the "Moreover" part of (4), the second term and the third term of the left hand side belong to $\operatorname{Diff}_{R}^{n}(I) R_{S}$. This implies $d(f) \in \operatorname{Diff}_{R}^{n}(I) R_{S}$.

This completes the proof for Lemma 1.1.2.1.

Corollary 1.1.2.2. Let $X$ be a variety over $k$. Then there exists a coherent sheaf $\mathcal{H o m}_{\mathcal{O}_{X}}\left(\mathcal{P}_{X}^{n}, \mathcal{O}_{X}\right) \stackrel{\sim}{\rightarrow} \mathcal{D}$ iff $f_{X}^{n}$ of the differential operators of degree $\leq n$ for $n \in \mathbb{Z}_{\geq 0}$ such that for any affine open subset $U=\operatorname{Spec} R \subset X$ we have

$$
\mathcal{H o m}_{\mathcal{O}_{X}}\left(\mathcal{P}_{X}^{n}, \mathcal{O}_{X}\right)(U)=\operatorname{Hom}_{R}\left(P_{R}^{n}, R\right) \stackrel{\sim}{\rightarrow} \operatorname{Diff}_{R}^{n}=\mathcal{D}_{i f f}^{n}(U)
$$

and that for any point $x \in X$ we have a description of the stalk as

$$
\left\{\mathcal{H o m}_{\mathcal{O}_{X}}\left(\mathcal{P}_{X}^{n}, \mathcal{O}_{X}\right)\right\}_{x}=\operatorname{Hom}_{\mathcal{O}_{X, x}}\left(P_{\mathcal{O}_{X, x}}^{n}, \mathcal{O}_{X, x}\right) \stackrel{\sim}{\rightarrow} \operatorname{Diff}_{\mathcal{O}_{X, x}}^{n}=\left\{\mathcal{D} \text { iff } f_{X}^{n}\right\}_{x} .
$$

Moreover, for any closed point $x \in X$ we have a description of the completion of the stalk as

$$
\begin{array}{rlc}
\left\{\mathcal{H o m}_{\mathcal{O}_{X}}\left(\mathcal{P}_{X}^{n}, \mathcal{O}_{X}\right)\right\}_{x} \otimes_{\mathcal{O}_{X, x}} \widehat{\mathcal{O}_{X, x}} & & \left\{\mathcal{D}_{i f f}^{n}\right\}_{x} \otimes_{\mathcal{O}_{X, x}} \widehat{\mathcal{O}_{X, x}} \\
\| & \stackrel{\|}{\rightarrow} & \operatorname{Diff}_{\widehat{\mathcal{O}_{X, x}}}^{n}
\end{array}
$$

Proof. This follows immediately from Lemma 1.1.2.1.

\section{§1.2. Basic properties of differential operators on a variety smooth over $k$}

The purpose of this section is to discuss some basic properties of differential operators on a variety $W$ smooth over $k$. 
Accordingly, we denote by $R$ the coordinate ring of an affine open subset Spec $R \subset W$, or its localization by some multiplicative set.

\subsubsection{Explicit description of differential operators with respect} to a regular system of parameters.

Definition 1.2.1.1. We say $\left(x_{1}, \ldots, x_{d}\right)$ with $d=\operatorname{dim} W$ is a regular system of parameters for $R$ if $\left\{d x_{\alpha}=\left(1 \otimes x_{\alpha}-x_{\alpha} \otimes 1 \bmod I\right) ; \alpha=1, \ldots, d\right\}$ forms a basis for the module of differentials $\Omega_{R / k}^{1}$ as an $R$-module, i.e.,

$$
\Omega_{R / k}^{1}=I / I^{2}=\bigoplus_{\alpha=1}^{d} R d x_{\alpha} \cong R^{d},
$$

where $I \subset R \otimes_{k} R$ is the kernel of the multiplication map $\mu: R \otimes_{k} R \rightarrow R$ (cf. Definition 1.1.1.1).

(Note that in the case where $R$ is the local ring associated to a closed point $P \in W$ such a regular system of parameters always exists, and that in the case where $R$ represents the coordinate ring of an affine open subset $\operatorname{Spec} R \subset W$ such a regular system of parameters exists by "shrinking" $\operatorname{Spec} R$ if necessary.)

Lemma 1.2.1.2. Suppose we have a regular system of parameters $\left(x_{1}, \ldots, x_{d}\right)$ for $R$ with $d=\operatorname{dim} W$. Then we have the following:

(1) We have a family of maps $\left\{\partial_{X^{J}}: R \rightarrow R ; J \in \mathbb{Z}_{\geq 0}^{d}\right\}$ such that

(i) $\partial_{X^{J}}\left(X^{I}\right)=\left(\begin{array}{l}I \\ J\end{array}\right) X^{I-J}$ for any $I \in \mathbb{Z}_{\geq 0}^{d}$, and that

(ii) $\left\{\partial_{X^{J}} ;|J| \leq n\right\}$ forms a basis of $\operatorname{Diff}_{R}^{n}$ for any $n \in \mathbb{Z}_{\geq 0}$, i.e.,

$$
\operatorname{Diff}_{R}^{n}=\bigoplus_{|J| \leq n} R \partial_{X^{J}} \cong R^{\left(\begin{array}{c}
n+d \\
n
\end{array}\right)} .
$$

(2) Let $\widehat{R}$ be the completion of $R$ with respect to a maximal ideal $\mathfrak{m}$ (corresponding to a closed point $P \in W)$. Then the $\widehat{R}$-module $\operatorname{Diff}_{\widehat{R}}^{n} \stackrel{\sim}{\rightarrow} \operatorname{Diff}_{R}^{n} \otimes_{R} \widehat{R}$ is free of rank $\left(\begin{array}{c}n+d \\ n\end{array}\right)$, having a basis $\left\{\partial_{X^{J}} ;|J| \leq n\right\}$ of the differential operators of degree $\leq n$. The differential operators are continuous with respect to the $\mathfrak{m}$-adic topology.

Set $y_{i}=x_{i}-\alpha_{i}$ for $1 \leq i \leq d$, where $\alpha_{i} \in k$, so that $Y=\left(y_{1}, \ldots, y_{d}\right)$ is a regular system of parameters for $R_{\mathfrak{m}}$. Then for any $f=\sum c_{I} Y^{I} \in$ $k\left[\left[y_{1}, \ldots, y_{d}\right]\right]=\widehat{R}$, we have

$$
\partial_{J}(f)=\partial_{J}\left(\sum c_{I} Y^{I}\right)=\sum c_{I} \partial_{J}\left(Y^{I}\right)=\sum c_{I}\left(\begin{array}{l}
I \\
J
\end{array}\right) Y^{I-J}
$$


where $\partial_{J}$ is the abbreviated notation for $\partial_{X^{J}}$.

(3) We have the generalized product rule

$$
\partial_{J}(f g)=\sum_{K+L=J} \partial_{K}(f) \partial_{L}(g) \quad \text { for } f, g \in R(\text { or } \widehat{R}) .
$$

Proof.

(1) We refer the reader to Theorem (16.11.2) in EGA IV [Gro67].

(2) Observe that a differential operator (of degree $\leq n$ ) is continuous with respect to the $\mathfrak{m}$-adic topology, a fact which easily follows, e.g., from the Leibnitz rule (of degree $n$ ). Note that $\partial_{Y^{J}}(f)=\partial_{X^{J}}(f)$ for any $J \in$ $\mathbb{Z}_{\geq 0}^{d}$ and $f \in \widehat{R}$ by definition of $Y=\left(y_{1}, \ldots, y_{d}\right)$. The rest is a direct consequence of (1).

(3) In order to check the generalized product rule, it suffices to check it for the localization $R_{\mathfrak{m}}$ for any maximal ideals of $R$. In order to check it for the localization $R_{\mathfrak{m}}$, it suffices to check it for its completion $\widehat{R}$ with respect to $\mathfrak{m}$.

By choosing a regular system of parameters $Y=\left(y_{1}, \ldots, y_{d}\right)$ for $R_{\mathfrak{m}}$ as in (2), we can identify $\widehat{R}$ with the power series ring $k\left[\left[y_{1}, \ldots, y_{d}\right]\right]$. Thus we have only to check (3) for the power series ring $k\left[\left[y_{1}, \ldots, y_{d}\right]\right]$. By (2), it is also clear that we have only to check it for the case of one variable, i.e., $d=1$ with $y_{1}=y$ and that we may even assume $f$ and $g$ are powers of $y$, i.e., $f=y^{a}$ and $g=y^{b}$. Then we have

$$
\begin{aligned}
\partial_{X^{J}}(f g) & =\partial_{Y^{J}}(f g)=\partial_{y^{n}}\left(y^{a} y^{b}\right)=\partial_{y^{n}}\left(y^{a+b}\right) \\
& =\left(\begin{array}{c}
a+b \\
n
\end{array}\right) y^{a+b-n}=\left(\sum_{l+m=n}\left(\begin{array}{c}
a \\
l
\end{array}\right)\left(\begin{array}{c}
b \\
m
\end{array}\right)\right) y^{a+b-n} \\
& =\sum_{l+m=n}\left(\begin{array}{c}
a \\
l
\end{array}\right) x^{a-l}\left(\begin{array}{c}
b \\
m
\end{array}\right) x^{b-m}=\sum_{K+L=J} \partial_{K}(f) \partial_{L}(g),
\end{aligned}
$$

which verifies the generalized product rule.

This completes the proof of Lemma 1.2.1.2.

Remark 1.2.1.3.

(1) It is easy to see that we have a relation

$$
\left(\partial_{x_{1}}\right)^{j_{1}} \circ\left(\partial_{x_{2}}\right)^{j_{2}} \circ \cdots \circ\left(\partial_{x_{d}}\right)^{j_{d}}=J ! \cdot \partial_{X^{J}}
$$

where $J !=\prod_{\alpha=1}^{d} j_{\alpha}$ ! in the multi-index notation. 
In characteristic zero, since $J ! \neq 0$, the above relation implies that all the differential operators are expressed as (the linear combinations over $R$ of) the composites of the differential operators of degree $\leq 1$, e.g., $R$-homomorphisms and $\partial_{x_{1}}, \ldots, \partial_{x_{d}}$.

In positive characteristic $\operatorname{char}(k)=p>0$, however, $J$ ! could well be equal to 0 and hence we start seeing the differential operators of higher order which cannot be expressed as (the linear combinations over $R$ of) the composites of differential operators of lower degrees, e.g.,

$$
\partial_{x_{\alpha}^{p^{1}}}, \partial_{x_{\alpha}^{p^{2}}}, \ldots, \partial_{x_{\alpha}^{p e}}, \ldots \quad \text { for } \alpha=1, \ldots, d \text { and } e \in \mathbb{Z}_{>0} .
$$

It is these operators which play a crucial role in positive characteristic.

(2) The following observation comes in handy when we compute the binomial coefficients in positive characteristic $\operatorname{char}(k)=p>0$ :

Let $i=\sum_{e} a_{e} p^{e}$ and $j=\sum_{e} b_{e} p^{e}$ be the expressions of the integers $i, j \in$ $\mathbb{Z}_{\geq 0}$ as $p$-adic numbers with $0 \leq a_{e}, b_{e}<p$. Then we have

$$
\left(\begin{array}{l}
i \\
j
\end{array}\right)=\prod_{e}\left(\begin{array}{l}
a_{e} \\
b_{e}
\end{array}\right) \bmod p .
$$

The identity follows immediately from the observation that, in $(\mathbb{Z} / p \mathbb{Z})[x]$, the number $\left(\begin{array}{l}i \\ j\end{array}\right)$ is the coefficient of $x^{j}=\prod_{e} x^{b_{e} p^{e}}$ in the polynomial $(1+x)^{i}=$ $\prod_{e}(1+x)^{a_{e} p^{e}}=\prod_{e}\left(1+x^{p^{e}}\right)^{a_{e}}$, which can be computed as the product of the coefficients $\left(\begin{array}{l}a_{e} \\ b_{e}\end{array}\right)$ of $x^{b_{e} p^{e}}$ in $\left(1+x^{p^{e}}\right)^{a_{e}}$.

\subsubsection{Logarithmic differential operators.}

Definition 1.2.2.1. $\quad$ Let $E$ be a simple normal crossing divisor on Spec $R$, and $I_{E} \subset R$ its defining ideal. We define the set $\operatorname{Diff}_{R, E}^{n}$ of the logarithmic differential operators of degree $\leq n$ on $R$ with respect to $E$ by

$$
\operatorname{Diff}_{R, E}^{n}=\left\{d \in \operatorname{Diff}_{R}^{n} ; d\left(I_{E}^{t}\right) \subset I_{E}^{t} \forall t \in \mathbb{Z}_{\geq 0}\right\} .
$$

Lemma 1.2.2.2. $\quad$ Suppose we have a regular system of parameters $\left(x_{1}, \ldots, x_{d}\right)$ for $R$ with $d=\operatorname{dim} W$, and a simple normal crossing divisor $E$ defined by $I_{E}=\left(\prod_{i=1}^{m} x_{i}\right)$ for some $1 \leq m \leq d$. Then we have the following:

(1) The R-module Diff $R_{R, E}^{n}$ is free of rank $\left(\begin{array}{c}n+d \\ n\end{array}\right)$. It has a basis $\left\{X^{J_{E}} \partial_{X^{J}} ;|J| \leq\right.$ $n\}$ (cf. Lemma 1.2.1.2 (1)), where $J_{E}=\left(j_{1}, \ldots, j_{m}, 0, \ldots, 0\right)$ for $J=\left(j_{1}, \ldots\right.$, $\left.j_{m}, j_{m+1}, \ldots, j_{d}\right)$. Thus we have

$$
\operatorname{Diff}_{R, E}^{n}=\bigoplus_{|J| \leq n} R X^{J_{E}} \partial_{X^{J}} \cong R^{\left(\begin{array}{c}
n+d \\
n
\end{array}\right)}
$$


(2) We have the logarithmic version of the generalized product formula

$$
X^{J_{E}} \partial_{J}(f g)=\sum_{K+L=J} X^{K_{E}} \partial_{K}(f) X^{L_{E}} \partial_{L}(g) \quad \text { for } f, g \in R \quad(\text { or } \widehat{R}) .
$$

Proof. This follows immediately from Lemma 1.2.1.2 and Definition 1.2.2.1.

Remark 1.2.2.3. Historically, after [Hir70] [Oda73] the development of techniques using the differential operators, including the logarithmic ones, in the study of singularities and their resolutions could be attributed to Jean Giraud (cf. [Gir74] [Gir75]). (We would like to thank the referee for bringing the historical impact of Giraud's work to our attention.) Personally, however, we first learned the explicit use of the logarithmic differential operators in the context of resolution of singularities from [Cos87] and [BM97]. It is worthwhile noting that even when we look at the existing algorithms which only use the usual differential operators on the surface (e.g. [Vil89] [Vil92] [EV00] [EH02] [Wło05]), one could implicitly observe the use of the logarithmic ones in the proof of Giraud's lemma (cf. [Gir74]) they depend upon. We invite the reader to look at [Bie04] [Bie05] and [BM03] [BM07] for the discussions on how the use of the logarithmic differential operators, in contrast to the use of the usual ones, affects the functorial properties of the algorithm, and even the formulation of the problem of resolution of singularities.

The use of the logarithmic differential operators is a "must" for our algorithm to function, as we will see in Parts III and IV, and is recognized as one of the key ingredients of the IFP from the very beginning of its conception.

1.2.3. Relation with multiplicity. We end this section by pointing out a basic relation between the multiplicity (order) and the differential operators in the form of a lemma. It is because of this basic relation that the differential operators play a key role in constructing an algorithm for resolution of singularities, where the order function constitutes a fundamental invariant.

Lemma 1.2.3.1. Let $I \subset R$ be an ideal. Let $P \in \operatorname{Spec} R$ be a point. Then

$$
\operatorname{ord}_{P}(I) \geq n \Longleftrightarrow P \in V\left(\operatorname{Diff}_{R}^{n-1}(I)\right) .
$$

In particular, the order function $\operatorname{ord}_{*}(I): \operatorname{Spec} R \rightarrow \mathbb{Z}_{\geq 0}$ is upper semicontinuous. 
Proof. $\quad$ First we show the equivalence in the case when $P$ is a closed point. Let $\mathfrak{m} \subset R$ be the maximal ideal corresponding to the closed point $P$. Let $\widehat{R}$ be the completion of $R$ with respect to $\mathfrak{m}$. Note that $\operatorname{ord}_{P}(I)=\operatorname{ord}_{P}(\widehat{I})$, where $\widehat{I}=I \widehat{R}$. On the other hand, since $\operatorname{Diff}_{\widehat{R}}^{n-1}(\widehat{I})=\operatorname{Diff}_{R}^{n}(I) \widehat{R}$ by Lemma 1.1.2.1 (7) and since $\widehat{R}$ is faithfully flat over $R$, we have $\operatorname{Diff}_{\widehat{R}}^{n-1}(\widehat{I}) \cap R=$ $\operatorname{Diff}_{R}^{n}(I)$. Thus we have only to show the equivalence at the level of completion. Choose a regular system of parameters $\left(x_{1}, \ldots, x_{d}\right)$ for $R_{\mathfrak{m}}$. Identify $\widehat{R}$ with the power series ring $k\left[\left[x_{1}, \ldots, x_{d}\right]\right]$. By definition, $\operatorname{ord}_{P}(\widehat{I}) \geq n$ if and only if, given $f=\sum_{J} c_{J} X^{J} \in \widehat{I} \subset k\left[\left[x_{1}, \ldots, x_{d}\right]\right]$ with $c_{J} \in k$, we have $c_{J}=0$ for any $J$ with $|J|<n$. By Lemma 1.2.1.2 (2), the last condition is equivalent to saying $\partial_{X^{K}}(f) \subset \widehat{\mathfrak{m}}$ for any $f \in \widehat{I}$ and $K$ with $|K|<n$. Since $\left\{\partial_{X^{K}} ;|K|<n\right\}$ generates Diff $\widehat{R}_{\widehat{R}}^{n-1}$ as an $\widehat{R}$-module (cf. Lemma 1.2.1.2 (2)), this condition is equivalent to $\operatorname{Diff}_{\widehat{R}}^{n-1}(\widehat{I}) \subset \widehat{\mathfrak{m}}$, i.e., $P \in V\left(\operatorname{Diff}_{\widehat{R}}^{n-1}(\widehat{I})\right)$. Therefore, we conclude

$$
\operatorname{ord}_{P}(\widehat{I}) \geq n \Longleftrightarrow P \in V\left(\operatorname{Diff}_{\widehat{R}}^{n-1}(\widehat{I})\right) .
$$

From the above argument it follows that the equivalence asserted in the lemma holds for a closed point and that the order function is upper semicontinuous if we restrict ourselves to the space of the maximal ideals $\mathfrak{m}$-Spec $R$.

It is then straightforward to see that the same equivalence holds for an arbitrary point in Spec $R$ and that the order function is upper semi-continuous over $\operatorname{Spec} R$.

This completes the proof of Lemma 1.2.3.1.

\section{$\S 1.3$. Ideals generated by the $p^{e}$-th power elements}

In this section, we denote by $k$ an algebraically closed field of $\operatorname{char}(k)=$ $p>0$.

The purpose of this section is to give a characterization of the ideals generated by $p^{e}$-th power elements, fixing $e \in \mathbb{Z}_{\geq 0}$, as the ideals invariant under the action of the set of differential operators of degree $\leq p^{e}-1$.

We denote by $R$ the coordinate ring of an affine open subset $\operatorname{Spec} R$ of a variety $W$ smooth over $k$, or its localization at a maximal ideal. We denote by $\widehat{R}$ the completion of $R$ with respect to a maximal ideal of $R$.

\subsubsection{Characterization in terms of the differential operators.}

Definition 1.3.1.1. Fix a nonnegative integer $e \in \mathbb{Z}_{\geq 0}$. We denote the $e$-th power of the Frobenius map by

$$
F^{e}: R \rightarrow R
$$


i.e., $F^{e}(r)=r^{p^{e}}$ for $r \in R$. We use the same symbol $F^{e}$ for the $e$-th power of the Frobenius map of the localization $R_{S}$ or the completion $\widehat{R}$ by abuse of notation if there is no chance of confusion.

Proposition 1.3.1.2. $\quad$ Let $I \subset R$ be an ideal. Fix a nonnegative integer $e \in \mathbb{Z}_{\geq 0}$. Then the following conditions are equivalent:

(i) The ideal $I$ is generated by the $p^{e}$-th power elements, i.e., $I=\left(I \cap F^{e}(R)\right)$.

(ii) The ideal I is invariant under the action of the set of the differential operators of degree $\leq p^{e}-1$, i.e., $I=\operatorname{Diff}_{R}^{p^{e}-1}(I)$.

Moreover, the equivalence of conditions (i) and (ii) also holds over the completion $\widehat{R}$.

Before beginning the proof of Proposition 1.3.1.2, we remark a couple of facts in the form of a lemma.

Lemma 1.3.1.3. $\quad$ Let $R^{\prime}$ denote the localization $R_{S}$ with respect to a multiplicative set $S \subset R$ or the completion $\widehat{R}$ with respect to a maximal ideal $\mathfrak{m} \subset R$. Then we have

(1) $R \otimes_{F^{e}(R)} F^{e}\left(R^{\prime}\right)=R^{\prime}$,

(2) $\left\{I \cap F^{e}(R)\right\} R^{\prime}=\left\{I R^{\prime} \cap F^{e}\left(R^{\prime}\right)\right\} R^{\prime}$.

Proof.

(1) When $R^{\prime}=R_{S}$, the assertion is clear since $R \otimes_{F^{e}(R)} F^{e}\left(R_{S}\right)=$ $R F^{e}\left(R_{S}\right)=R_{S}$. When $R^{\prime}=\widehat{R}$, we see that $R \otimes_{F^{e}(R)} F^{e}(\widehat{R})$ and $\widehat{R}$ are the completions of $R$ with respect to the topologies defined by $\left\{F^{e}\left(\mathfrak{m}^{n}\right) R\right\}_{n \in \mathbb{Z}_{>0}}$ and $\left\{\mathfrak{m}^{n}\right\}_{n \in \mathbb{Z}_{>0}}$ respectively. It is easy to see that these two topologies coincide.

(2) Since $I \cap F^{e}(R) \subset I R^{\prime} \cap F^{e}\left(R^{\prime}\right)$, we have the inclusion $\left\{I \cap F^{e}(R)\right\} R^{\prime} \subset$ $\left\{I R^{\prime} \cap F^{e}\left(R^{\prime}\right)\right\} R^{\prime}$. In order to see the opposite inclusion, using the fact that $F^{e}\left(R^{\prime}\right)$ is flat over $F^{e}(R)$, we observe

$$
\begin{aligned}
\left\{I \cap F^{e}(R)\right\} R^{\prime} & \supset\left\{I \cap F^{e}(R)\right\} F^{e}\left(R^{\prime}\right)=\left\{I \cap F^{e}(R)\right\} \otimes_{F^{e}(R)} F^{e}\left(R^{\prime}\right) \\
& =\left\{I \otimes_{F^{e}(R)} F^{e}\left(R^{\prime}\right)\right\} \cap\left\{F^{e}(R) \otimes_{F^{e}(R)} F^{e}\left(R^{\prime}\right)\right\} \\
& =\left\{I \otimes_{R} R \otimes_{F^{e}(R)} F^{e}\left(R^{\prime}\right)\right\} \cap F^{e}\left(R^{\prime}\right) \\
& =\left\{I \otimes_{R} R^{\prime}\right\} \cap F^{e}\left(R^{\prime}\right)=I R^{\prime} \cap F^{e}\left(R^{\prime}\right),
\end{aligned}
$$

which implies the desired inclusion. 
This completes the proof of Lemma 1.3.1.3.

\section{Proof of Proposition 1.3.1.2.}

Step 1. Reduction to the case over the completion $\widehat{R}$.

Firstly note that two ideals of $R$ coincide if their localizations or even completions coincide at any maximal ideal $\mathfrak{m}$ of $R$. Thus it suffices to show the conditions

$$
I \widehat{R_{\mathfrak{m}}}=\left(I \cap F^{e}(R)\right) \widehat{R_{\mathfrak{m}}} \quad \text { and } \quad I \widehat{R_{\mathfrak{m}}}=\operatorname{Diff}_{R}^{p^{e}-1}(I) \widehat{R_{\mathfrak{m}}}
$$

are equivalent for any maximal ideal $\mathfrak{m} \subset R$. Secondly note that

$$
\begin{aligned}
& \left.\left(I \cap F^{e}(R)\right) \widehat{R_{\mathfrak{m}}}=\left\{I \widehat{R_{\mathfrak{m}}} \cap F^{e}\left(\widehat{R_{\mathfrak{m}}}\right)\right\} \widehat{R_{\mathfrak{m}}} \quad \text { (by Lemma 1.3.1.3 }(2)\right), \\
& \left.\operatorname{Diff}_{R}^{p^{e}}-1(I) \widehat{R_{\mathfrak{m}}}=\operatorname{Diff}_{\widehat{R_{\mathfrak{m}}}}^{p^{e}-1}\left(I \widehat{R_{\mathfrak{m}}}\right) \quad \text { (by Lemma 1.1.2.1 }(7)\right) \text {. }
\end{aligned}
$$

Therefore, it suffices to show the equivalence of the conditions in the case over $\widehat{R}=\widehat{R_{\mathfrak{m}}}$. In the following consideration, we identify $\widehat{R}$ with the power series ring $k\left[\left[x_{1}, \ldots, x_{d}\right]\right]$ (by choosing a regular system of parameters $\left(x_{1}, \ldots, x_{d}\right)$ for $\left.R_{\mathfrak{m}}\right)$.

Step 2. Verification of the implication (i) $\Longrightarrow$ (ii).

We obviously have $\widehat{I} \subset \operatorname{Diff}_{\widehat{R}}^{p^{e}-1}(\widehat{I})$. Thus we have only to show $\widehat{I} \supset \operatorname{Diff}_{\widehat{R}}^{p^{e}-1}(\widehat{I})$ assuming condition (i). By Lemma 1.2.1.2 (2), the set $\left\{\partial_{X^{J}} ;|J| \leq p^{e}-1\right\}$ generates Diff $p_{\widehat{R}}^{p^{e}-1}$ as an $\widehat{R}$-module. Therefore, it suffices to check $\partial_{X^{J}}(f) \in \widehat{I}$ for any $f \in \widehat{I}$ and $\partial_{X^{J}}$ with $|J| \leq p^{e}-1$. By assuming condition (i), we may assume $\widehat{I}=\left(\left\{r_{\lambda}^{p^{e}} ; r_{\lambda} \in \widehat{R}\right\}_{\lambda \in \Lambda}\right)$ so that we can write $f=\sum_{\lambda \in \Lambda} a_{\lambda} r_{\lambda}^{p^{e}}$ with $a_{\lambda} \in \widehat{R}$. We compute via the generalized product rule

$$
\begin{aligned}
\partial_{X^{J}}(f) & =\partial_{X^{J}}\left(\sum_{\lambda \in \Lambda} a_{\lambda} r_{\lambda}^{p^{e}}\right)=\sum_{\lambda \in \Lambda} \partial_{X^{J}}\left(a_{\lambda} r_{\lambda}^{p^{e}}\right) \\
& =\sum_{\lambda \in \Lambda}\left\{\sum_{K+L=J} \partial_{X^{K}}\left(a_{\lambda}\right) \partial_{X^{L}}\left(r_{\lambda}^{p^{e}}\right)\right\}=\sum_{\lambda \in \Lambda} \partial_{X^{J}}\left(a_{\lambda}\right) r_{\lambda}^{p^{e}} \in I .
\end{aligned}
$$

Note that, in order to obtain the last equality, we use the fact that $\partial_{X^{L}}\left(r_{\lambda}^{p^{e}}\right)=0$ unless $L=0$. In fact, if $r_{\lambda}=\sum_{J} c_{J} X^{J} \in k\left[\left[x_{1}, \ldots, x_{d}\right]\right]$, then, by Lemma 1.2.1.2 (2), we have

$$
\partial_{X^{L}}\left(r_{\lambda}^{p^{e}}\right)=\partial_{X^{L}}\left(\sum_{J} c_{J}^{p^{e}} X^{p^{e} J}\right)=\sum_{J} c_{J}^{p^{e}} \partial_{X^{L}}\left(X^{p^{e} J}\right)=\sum_{J} c_{J}^{p^{e}}\left(\begin{array}{c}
p^{e} J \\
L
\end{array}\right) X^{p^{e} J-L} .
$$


Since $\left(\begin{array}{c}p^{e} J \\ L\end{array}\right)=\prod_{i=1}^{d}\left(\begin{array}{c}p^{e} j_{i} \\ l_{i}\end{array}\right)$, and since $\left(\begin{array}{c}p^{e} j_{i} \\ l_{i}\end{array}\right)=0 \bmod p$ unless $l_{i}=0$ because $l_{i} \leq|L| \leq|J| \leq p^{e}-1$, we conclude $\partial_{X^{L}}\left(r_{\lambda}^{p^{e}}\right)=0$ unless $L=0$.

This completes the verification of the implication (i) $\Longrightarrow$ (ii).

Step 3. Verification of the implication (ii) $\Longrightarrow(\mathrm{i})$.

We obviously have $\widehat{I} \supset\left(\widehat{I} \cap F^{e}(\widehat{R})\right)$. Thus we have only to show $\widehat{I} \subset\left(\widehat{I} \cap F^{e}(\widehat{R})\right)$ assuming condition (ii). First note that, setting $\Gamma=\left\{0,1, \ldots, p^{e}-1\right\}^{d}$, we can express any $f \in \widehat{R}=k\left[\left[x_{1}, \ldots, x_{d}\right]\right]$ in the form

$$
f=\sum_{M \in \Gamma} a_{M}^{p^{e}} X^{M}
$$

where the set of coefficients $\left\{a_{M}^{p^{e}} ; a_{M} \in \widehat{R}\right\}_{M \in \Gamma}$ is uniquely determined.

It suffices to show that, given $f \in \widehat{I}$ and its expression as above, we have $\left\{a_{M}^{p^{e}} ; a_{M} \in \widehat{R}\right\}_{M \in \Gamma} \subset \widehat{I}$, which implies $f \in\left(\widehat{I} \cap F^{e}(\widehat{R})\right)$.

We derive a contradiction assuming $\left\{a_{M}^{p^{e}} ; a_{M} \in \widehat{R}\right\}_{M \in \Gamma} \not \subset \widehat{I}$. Set

$$
N=\max \left\{M \in \Gamma ; a_{M}^{p^{e}} \notin \widehat{I}\right\},
$$

where the maximum is taken with respect to the lexicographical order on $\Gamma$. We compute via the generalized product rule

$$
\begin{aligned}
\widehat{I} & =\operatorname{Diff}_{\widehat{R}}^{p^{e}-1}(\widehat{I}) \ni \partial_{X^{N}}\left(f-\sum_{M>N} a_{M}^{p^{e}} X^{M}\right)=\sum_{M \leq N} \partial_{X^{N}}\left(a_{M}^{p^{e}} X^{M}\right) \\
& =\sum_{M \leq N} \sum_{K+L=N} \partial_{X^{K}}\left(a_{M}^{p^{e}}\right) \partial_{X^{L}}\left(X^{M}\right)=\sum_{M \leq N} a_{M}^{P^{e}} \partial_{X^{N}}\left(X^{M}\right)=a_{N}^{p^{e}} .
\end{aligned}
$$

Note that, by the same argument as in Step 2 of this proof, we see $\partial_{X^{K}}\left(a_{M}^{p^{e}}\right)=0$ unless $K=0$. This is used to obtain the second last equality. Note also that $\left(\begin{array}{l}M \\ N\end{array}\right)=0$ if $M<N$. Indeed, if $M<N$, there exists $1 \leq i_{o} \leq d$ such that $m_{i_{o}}<n_{i_{o}}$, which implies $\left(\begin{array}{c}m_{i_{o}} \\ n_{i_{o}}\end{array}\right)=0$ (cf. 0.9.3) and hence $\left(\begin{array}{c}M \\ N\end{array}\right)=\prod_{i=1}^{d}\left(\begin{array}{c}m_{i} \\ n_{i}\end{array}\right)=0$. Thus $\partial_{X^{N}}\left(X^{M}\right)=0$ if $M<N$. This is used to obtain the last equality. Therefore, we have $a_{N}^{p^{e}} \in \widehat{I}$, contradicting the choice of $N$. This completes the verification of the implication (ii) $\Longrightarrow$ (i).

This completes the proof of Proposition 1.3.1.2.

We end this section by stating a lemma, which is proved in the same spirit as the proof of Proposition 1.3.1.2 and is of interest on its own.

Lemma 1.3.1.4. Let $\widehat{R}$ be the completion of $R$ with respect to a maximal ideal $\mathfrak{m}$ of $R$, and $e \in \mathbb{Z}_{\geq 0}$ a nonnegative integer. Then

$$
R \cap F^{e}(\widehat{R})=F^{e}(R)
$$


In other words, if $r \in R$ has its $p^{e}$-th root $f$ within $\widehat{R}$, then $f$ actually belongs to $R$.

Proof. $\quad$ Since $\widehat{R}$ is faithfully flat over $R$, so is $F^{e}(\widehat{R})$ over $F^{e}(R)$. Applying Theorem 7.5 in [Mat86] to the $F^{e}(R)$-module $R / F^{e}(R)$, we see that the natural map

$$
R / F^{e}(R) \rightarrow\left(R / F^{e}(R)\right) \otimes_{F^{e}(R)} F^{e}(\widehat{R})
$$

is injective. On the other hand, using Lemma 1.3.1.3, we analyze the target of the above map to be

$$
\begin{aligned}
\left(R / F^{e}(R)\right) \otimes_{F^{e}(R)} F^{e}(\widehat{R}) & =\left\{R \otimes_{F^{e}(R)} F^{e}(\widehat{R})\right\} /\left\{F^{e}(R) \otimes_{F^{e}(R)} F^{e}(\widehat{R})\right\} \\
& =\widehat{R} / F^{e}(\widehat{R}) .
\end{aligned}
$$

That is to say, we conclude that the map $R / F^{e}(R) \rightarrow \widehat{R} / F^{e}(\widehat{R})$ is injective, and hence that $R \cap F^{e}(\widehat{R})=F^{e}(R)$.

This completes the proof of Lemma 1.3.1.4.

\section{Chapter 2. Idealistic Filtration}

The purpose of this chapter is to introduce the notion of an idealistic filtration, which is the main language to describe our program toward constructing an algorithm for resolution of singularities, and establish its fundamental properties.

We develop our argument over a ring $R$, which is assumed to be the coordinate ring of an affine open subset of a nonsingular variety $W$ over $k$, or its localization, or its completion with respect to a maximal ideal. That is to say, more geometrically speaking, we carry out our analysis over an affine open subset of a nonsingular variety $W$, or over a stalk, or over the analytic structure at a closed point. Since the main operations on an idealistic filtration, such as the operations of taking the $\mathfrak{D}$-saturation and $\mathfrak{R}$-saturation, are compatible with localization and completion (for an idealistic filtration of r.f.g. type), it is immediate to extend the (analytically) local analysis of this chapter to the global argument, which we will develop in the subsequent papers.

\section{§2.1. Idealistic filtration over a ring}

Let $R$ be the coordinate ring of an affine open subset of a nonsingular variety $W$ over $k$, or its localization, or its completion with respect to a maximal ideal. 


\subsubsection{Definitions.}

\section{Definition 2.1.1.1.}

(1) Let $T \subset R \times \mathbb{R}$ be a subset. For $a \in \mathbb{R}$, we set $T_{a}=\{f \in R ;(f, a) \in T\}$.

(2) We call a subset $\mathbb{I} \subset R \times \mathbb{R}$ an idealistic filtration if it satisfies the following conditions:

$$
\left\{\begin{aligned}
\text { (o) } \mathbb{I}_{0}=R, \\
\text { (i) } \mathbb{I}_{a} \text { is an ideal of } R \text { for any } a \in \mathbb{R} \\
\quad\left(\mathbb{I}_{a} \text { is called the ideal of } \mathbb{I} \text { at level } a\right) \\
\text { (ii) } \mathbb{I}_{a} \mathbb{I}_{b} \subset \mathbb{I}_{a+b} \text { for any } a, b \in \mathbb{R}, \\
\text { (iii) } \mathbb{I}_{b} \supset \mathbb{I}_{a} \text { if } b \leq a .
\end{aligned}\right.
$$

(3) Let $T \subset R \times \mathbb{R}$ be a subset. We call the minimal idealistic filtration containing $T$ the idealistic filtration generated by $T$ and denote it by $G(T)$. If $\mathbb{I}=G(T)$, we call $T$ a set of generators for $\mathbb{I}$ (cf. Lemma 2.2.1.1 (2)).

When we want to emphasize the base ring $R$ over which $T$ generates the idealistic filtration, we write $G_{R}(T)$ inserting $R$ as a subscript.

(4) We say an idealistic filtration $\mathbb{I}$ is of r.f.g. type (short for rationally and finitely generated) if there exists a finite set $T \subset R \times \mathbb{Q} \subset R \times \mathbb{R}$ such that $\mathbb{I}=G(T)$.

(5) Let $T \subset R \times \mathbb{R}_{\geq 0}$ be a subset. Let $P \in \operatorname{Spec} R$ be a point. We define the multiplicity $\mu_{P}(T)$ of $T$ at $P$ to be

$$
\mu_{P}(T):=\inf \left\{\mu_{P}(f, a):=\frac{\operatorname{ord}_{P}(f)}{a} ;(f, a) \in T, a>0\right\} .
$$

Note that we set $\mu_{P}(f, 0)=\infty$ for any $f \in R$ by definition, while $\operatorname{ord}_{P}(0)=\infty$.

(6) Let $T \subset R \times \mathbb{R}$ be a subset. We define the support $\operatorname{Supp}(T)$ of $T$ to be

$$
\operatorname{Supp}(T)=\left\{P \in \operatorname{Spec} R ; \mu_{P}(T) \geq 1\right\} .
$$

Remark 2.1.1.2.

(1) It is straightforward to see that a subset $\mathbb{I} \subset R \times \mathbb{R}$ is an idealistic filtration if and only if it satisfies the following conditions:

$$
\left\{\begin{array}{l}
\text { (o) }(f, 0) \in \mathbb{I} \quad \forall f \in R, \quad(0, a) \in \mathbb{I} \quad \forall a \in \mathbb{R}, \\
\text { (i) }(f, a),(g, a) \in \mathbb{I} \Longrightarrow(f+g, a) \in \mathbb{I}, \\
r \in R,(f, a) \in \mathbb{I} \Longrightarrow(r f, a) \in \mathbb{I}, \\
\text { (ii) }(f, a),(h, b) \in \mathbb{I} \Longrightarrow(f h, a+b) \in \mathbb{I}, \\
\text { (iii) }(f, a) \in \mathbb{I}, b \leq a \Longrightarrow(f, b) \in \mathbb{I} .
\end{array}\right.
$$


We invite the reader to look at 0.2.3.1. in Chapter 0 for the motivation behind introducing the notion of an idealistic filtration.

(2) When $T=\mathbb{I} \subset R \times \mathbb{R}$ is an idealistic filtration, we define its multiplicity $\mu_{P}(\mathbb{I})$ at a point $P \in \operatorname{Spec} R$, and its support $\operatorname{Supp}(\mathbb{I})$ according to Definition 2.1.1.1 (5) and (6).

2.1.2. $\mathfrak{D}$-saturation. We define the notion of the differential saturation (which we call the $\mathfrak{D}$-saturation for short) of an idealistic filtration. Budding of an idea leading to the notion of $\mathfrak{D}$-saturation can be observed in the work of Giraud and Villamayor, where they discuss the enlargement, called the extension, of an ideal obtained by adding the partial derivatives of the elements in the ideal.

Definition 2.1.2.1. Let $\mathbb{I} \subset R \times \mathbb{R}$ be an idealistic filtration. We say $\mathbb{I}$ is $\mathfrak{D}$-saturated if it satisfies the following condition (differential):

$$
\text { (differential) }(f, a) \in \mathbb{I}, d \in \operatorname{Diff}_{R}^{t} \Longrightarrow(d(f), a-t) \in \mathbb{I} \text {. }
$$

(We refer the reader to Chapter 1 for the meaning of the notation Difft ${ }_{R}$.)

Let $\mathbb{I}$ be an idealistic filtration. We call the minimal $\mathfrak{D}$-saturated idealistic filtration containing $\mathbb{I}$ the differential saturation (or $\mathfrak{D}$-saturation for short) of $\mathbb{I}$, and denote it by $\mathfrak{D}(\mathbb{I})$ (cf. Lemma 2.2.1.1).

Let $E$ be a simple normal crossing divisor on $W$. Then using the logarithmic differential operators with respect to $E$ instead of the usual differential operators (cf. Definition 1.2.2.1), we consider the following condition $(\text { differential })_{E}$ :

$$
(\text { differential })_{E} \quad(f, a) \in \mathbb{I}, d \in \operatorname{Difft}_{R, E}^{t} \Longrightarrow(d(f), a-t) \in \mathbb{I} \text {. }
$$

Replacing condition (differential) with condition (differential) $E$, we obtain the

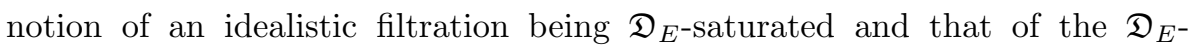
saturation.

2.1.3. $\Re$-saturation. We define the notion of the radical saturation (which we call the $\mathfrak{R}$-saturation for short) of an idealistic filtration. Note that, for an $\mathfrak{R}$-saturated idealistic filtration, we not only require that we can take the $n$-th root (radical) of an element within the idealistic filtration (if it exists within $R \times \mathbb{R}$ ) for any $n \in \mathbb{Z}_{>0}$, but also require the continuity by definition. 
Definition 2.1.3.1. $\quad$ Let $\mathbb{I} \subset R \times \mathbb{R}$ be an idealistic filtration. We say $\mathbb{I}$ is $\mathfrak{R}$-saturated if it satisfies the following conditions (radical) and (continuity):

$$
\begin{array}{ll}
\text { (radical) } & \left(f^{n}, n a\right) \in \mathbb{I}, f \in R, n \in \mathbb{Z}_{>0} \quad \Longrightarrow(f, a) \in \mathbb{I} \\
\text { (continuity) } & \left\{\left(f, a_{l}\right)\right\} \subset \mathbb{I} \text { with } \lim _{l \rightarrow \infty} a_{l}=a \Longrightarrow(f, a) \in \mathbb{I} .
\end{array}
$$

Let $\mathbb{I}$ be an idealistic filtration. We call the minimal $\mathfrak{R}$-saturated idealistic filtration containing $\mathbb{I}$ the radical saturation (or $\mathfrak{R}$-saturation for short) of $\mathbb{I}$, and denote it by $\mathfrak{R}(\mathbb{I})$ (cf. Lemma 2.2.1.1).

\section{Remark 2.1.3.2.}

(1) We remark that, in positive characteristic $p=\operatorname{char}(k)>0$, if an idealistic filtration II satisfies the following condition (Frobenius), which is a priori slightly weaker than condition (radical), and condition (continuity), then it actually satisfies conditions (radical) and (continuity). Therefore, instead of checking conditions (radical) and (continuity) in order to show that a given idealistic filtration is $\mathfrak{R}$-saturated in positive characteristic, we could check conditions (Frobenius) and (continuity):

$$
\text { (Frobenius) } \quad\left(f^{p}, p a\right) \in \mathbb{I}, f \in R \Longrightarrow(f, a) \in \mathbb{I} .
$$

In fact, suppose we have $\left(f^{n}, n a\right) \in \mathbb{I}, f \in R$ and $n \in \mathbb{Z}_{>0}$. Take $e \in \mathbb{Z}_{>0}$ so that $p^{e}>n$, and take $r \in \mathbb{Z}_{\geq 0}$ with $0 \leq r<n$ so that $r \equiv p^{e} \bmod n$. Then

$$
\begin{aligned}
& \left(f^{n}, n a\right) \in \mathbb{I} \Longrightarrow\left(f^{p^{e}-r}, a \cdot\left(p^{e}-r\right)\right) \in \mathbb{I} \text { by condition (ii) in Remark 2.1.1.2 (1) } \\
& \Longrightarrow\left(f^{p^{e}}, a \cdot\left(p^{e}-r\right)\right) \in \mathbb{I} \quad \text { by condition (i) in Remark 2.1.1.2 (1) } \\
& \Longrightarrow\left(f, a \cdot\left(1-p^{-e} r\right)\right) \in \mathbb{I} \text { by condition (Frobenius) } \\
& \Longrightarrow(f, a) \in \mathbb{I} \quad \text { by condition (continuity) with } e \rightarrow \infty \text {. }
\end{aligned}
$$

(2) In view of condition (iii) in Remark 2.1.1.2 (1), requiring condition (continuity) is equivalent to requiring the following (left continuity):

(left continuity) $\left\{\left(f, a_{l}\right)\right\} \subset \mathbb{I}$ with $\left\{a_{l}\right\}$ increasing and $\lim _{l \rightarrow \infty} a_{l}=a \Longrightarrow(f, a) \in \mathbb{I}$.

In terms of the ideals of an idealistic filtration associated to the levels, condition (left continuity) translates into the condition

$$
\mathbb{I}_{a}=\bigcap_{b<a} \mathbb{I}_{b} \quad \forall a \in \mathbb{R} .
$$

When an idealistic filtration is of r.f.g. type, this condition can be checked rather easily. Therefore, we see that condition (continuity) is always satisfied for an idealistic filtration of r.f.g. type. See Corollary 2.3.2.3 for detail. 
2.1.4. Integral closure. We define the notion of the integral closure of an idealistic filtration, which is closely related to the notion of the $\mathfrak{R}$-saturation. In general, if an idealistic filtration is $\mathfrak{R}$-saturated, then it is integrally closed. In particular, for an idealistic filtration of r.f.g. type, where condition (continuity) is automatic, it is $\mathfrak{R}$-saturated if and only if it is integrally closed.

We also conclude in Corollary 2.3.2.7, through the argument showing the stability of r.f.g. type under $\mathfrak{R}$-saturation, that, for an idealistic filtration of r.f.g. type, the $\mathfrak{R}$-saturation and the integral closure coincide.

Definition 2.1.4.1. Let $\mathbb{I} \subset R \times \mathbb{R}$ be an idealistic filtration.

(1) We say an element $(f, a) \in R \times \mathbb{R}$ is integral over $\mathbb{I}$ if $f$ satisfies a monic equation of the form

$$
f^{n}+c_{1} f^{n-1}+\cdots+c_{n}=0 \quad \text { with }\left(c_{i}, i a\right) \in \mathbb{I} \text { for } i=1, \ldots, n .
$$

(2) We say $\mathbb{I}$ is integrally closed if it satisfies the following condition (ic):

(ic) $(f, a) \in R \times \mathbb{R}$ is integral over $\mathbb{I} \Longrightarrow(f, a) \in \mathbb{I}$.

Let II be an idealistic filtration. We call the minimal integrally closed idealistic filtration containing $\mathbb{I}$ the integral closure of $\mathbb{I}$, and denote it by $\operatorname{IC}(\mathbb{I})$ (cf. Lemma 2.2.1.1).

Remark 2.1.4.2. The notion of the integral closure is important in our program. However, since the $\mathfrak{R}$-saturation and the integral closure coincide for an idealistic filtration of r.f.g. type, and since almost all the idealistic filtrations we consider are of r.f.g. type, we seldom use the symbol IC(I) or the notion of the integral closure explicitly, and almost always use the notion of the $\mathfrak{R}$ saturation, which is denoted by $\mathfrak{R}(\mathbb{I})$.

2.1.5. B-saturation. We define the notion of the bi-saturation (which we call the $\mathfrak{B}$-saturation). For the purpose of extracting the intrinsic information toward a solution of the problem of resolution of singularities, we take various saturations of a given idealistic filtration (cf. 0.2.3.2.3.). It would be best if we could take an "optimal" one among such. In our algorithm, the $\mathfrak{B}$-saturation (or $\mathfrak{B}_{E}$-saturation) plays the role of the optimal saturation.

Definition 2.1.5.1. $\quad$ Let $\mathbb{I} \subset R \times \mathbb{R}$ be an idealistic filtration. We say $\mathbb{I}$ is $\mathfrak{B}$-saturated (resp. $\mathfrak{B}_{E}$-saturated) if it is both $\mathfrak{D}$-saturated (resp. $\mathfrak{D}_{E^{-}}$ saturated) and $\mathfrak{R}$-saturated. Given an idealistic filtration $\mathbb{I}$, we call the minimal 
$\mathfrak{B}$-saturated ( $\mathfrak{B}_{E}$-saturated) idealistic filtration containing $\mathbb{I}$ the $\mathfrak{B}$-saturation (resp. $\mathfrak{B}_{E}$-saturation) of $\mathbb{I}$, and denote it by $\mathfrak{B}(\mathbb{I})$ (resp. $\mathfrak{B}_{E}(\mathbb{I})$ ) (cf. Lemma 2.2.1.1).

Remark 2.1.5.2. While the existence of the $\mathfrak{B}$-saturation is as straightforward as the existence of the other saturations and integral closure, its explicit construction is quite remarkable, which we will see in Corollary 2.4.2.3. We describe the explicit construction of the other saturations and integral closure in Lemma 2.2.1.2.

\section{§2.2. Basic properties of an idealistic filtration}

In this section, we discuss some basic properties of an idealistic filtration over a ring. We use the same notation as in $\S 2.1$.

2.2.1. On generation, $\mathfrak{D}$-saturation, $\mathfrak{R}$-saturation, integral closure, and $\mathfrak{B}$-saturation. The next two lemmas discuss the existence and explicit construction of the idealistic filtration generated by a subset $T \subset R \times \mathbb{R}$, the $\mathfrak{D}$-saturation, $\mathfrak{R}$-saturation, integral closure, and $\mathfrak{B}$-saturation.

\section{Lemma 2.2.1.1.}

(1) The intersection $\bigcap_{\lambda \in \Lambda} \mathbb{I}_{\lambda} \subset R \times \mathbb{R}$ of a non-empty collection $\left\{\mathbb{I}_{\lambda}\right\}_{\lambda \in \Lambda}$ of idealistic filtrations is again an idealistic filtration. Moreover, if each $\mathbb{I}_{\lambda}$ is $\mathfrak{D}$-saturated (resp. $\mathfrak{D}_{E}$-saturated, $\mathfrak{R}$-saturated, integrally closed, $\mathfrak{B}$-saturated, $\mathfrak{B}_{E}$-saturated), then so is the intersection $\bigcap_{\lambda \in \Lambda} \mathbb{I}_{\lambda}$.

(2) Let $T \subset R \times \mathbb{R}$ be a subset. Then $G(T)$ exists (cf. Definition 2.1.1.1 (3)).

(3) Let $\mathbb{I}$ be an idealistic filtration. Then $\mathfrak{D}(\mathbb{I})$ (resp. $\mathfrak{D}_{E}(\mathbb{I}), \mathfrak{R}(\mathbb{I}), \operatorname{IC}(\mathbb{I}), \mathfrak{B}(\mathbb{I})$, $\mathfrak{B}_{E}(\mathbb{I})$ ) exists (cf. 2.1.2, 2.1.3, 2.1.4, 2.1.5).

\section{Proof.}

(1) It is clear from the definitions.

(2) Let $\mathcal{S}=\left\{\mathbb{I}_{\lambda} ; \mathbb{I}_{\lambda} \supset T\right\}$ be the collection of all the idealistic filtrations containing $T$. Note that $\mathcal{S}$ is non-empty, since $R \times \mathbb{R} \in \mathcal{S}$. Now it is clear that the intersection $\bigcap_{\mathbb{I}_{\lambda} \in \mathcal{S}} \mathbb{I}_{\lambda}$ is the minimal idealistic filtration containing $T$.

(3) Let $\mathcal{S}=\left\{\mathbb{I}_{\lambda} ; \mathbb{I}_{\lambda} \supset \mathbb{I}\right\}$ be the collection of all the $\mathfrak{D}$-saturated (resp. $\mathfrak{D}_{E}$-saturated, $\mathfrak{R}$-saturated, integrally closed, $\mathfrak{B}$-saturated, $\mathfrak{B}_{E}$-saturated) idealistic filtrations containing $\mathbb{I}$. Note that $\mathcal{S}$ is non-empty, since $R \times \mathbb{R} \in \mathcal{S}$. Now it is clear that the intersection $\bigcap_{\mathbb{I}_{\lambda} \in \mathcal{S}} \mathbb{I}_{\lambda}$ is the minimal $\mathfrak{D}$-saturated (resp. 
$\mathfrak{D}_{E}$-saturated, $\mathfrak{R}$-saturated, integrally closed, $\mathfrak{B}$-saturated, $\mathfrak{B}_{E}$-saturated) idealistic filtration containing $\mathbb{I}$.

This completes the proof of Lemma 2.2.1.1.

Lemma 2.2.1.2. Let $\mathbb{I}$ be an idealistic filtration generated by $T=\left\{\left(f_{\lambda}\right.\right.$, $\left.\left.a_{\lambda}\right)\right\} \subset R \times \mathbb{R}$, i.e., $\mathbb{I}=G(T)$.

(1) Define a subset $\mathbb{I}^{\prime} \subset R \times \mathbb{R}$ by setting

$$
\mathbb{I}_{a}^{\prime}=\left(\prod f_{\lambda}^{n_{\lambda}} ; n_{\lambda} \in \mathbb{Z}_{\geq 0}, \sum n_{\lambda} a_{\lambda} \geq a\right) \quad a \in \mathbb{R} .
$$

Then $\mathbb{I}^{\prime}$ is an idealistic filtration, and $\mathbb{I}^{\prime}=\mathbb{I}$. Note that, when $T=\emptyset$, we use the convention

$$
G(\emptyset)=(\{0\} \times \mathbb{R}) \cup\left(R \times \mathbb{R}_{\leq 0}\right) .
$$

(2) Let $\left(x_{1}, \ldots, x_{d}\right)$ be a regular system of parameters for $R$. Set

$$
T^{\prime}=\left\{\left(\partial_{X^{J}} f_{\lambda}, a_{\lambda}-|J|\right) ; J \in \mathbb{Z}_{\geq 0}^{d},\left(f_{\lambda}, a_{\lambda}\right) \in T\right\} .
$$

Then we have $\mathfrak{D}(\mathbb{I})=G\left(T^{\prime}\right)$.

Let $E$ be a simple normal crossing divisor, and say, $\left\{x_{1} \cdots x_{m}=0\right\}$ defines E for some $1 \leq m \leq d$. Set

$$
T_{E}^{\prime}=\left\{\left(X^{J_{E}} \partial_{X^{J}} f_{\lambda}, a_{\lambda}-|J|\right) ; J \in \mathbb{Z}_{\geq 0}^{d},\left(f_{\lambda}, a_{\lambda}\right) \in T\right\} .
$$

Then we have $\mathfrak{D}_{E}(\mathbb{I})=G\left(T_{E}^{\prime}\right)$. (We refer the reader to 1.2 .2 for the notation.)

(3) Define subsets $\mathbb{K}, \overline{\mathbb{K}} \subset R \times \mathbb{R}$ by

$$
\mathbb{K}_{a}=\left\{f \in R ; f^{n} \in \mathbb{I}_{n a} \text { for some } n \in \mathbb{Z}_{>0}\right\}, \quad \overline{\mathbb{K}}_{a}=\bigcap_{b<a} \mathbb{K}_{b} \quad(a \in \mathbb{R}) .
$$

Then $\overline{\mathbb{K}}$ is an idealistic filtration, and $\mathfrak{R}(\mathbb{I})=\overline{\mathbb{K}}$.

(4) Let $\mathbb{J} \subset R \times \mathbb{R}$ be the subset consisting of all the elements integral over $\mathbb{I}$. Then $\mathbb{J}$ is an idealistic filtration, and $\mathrm{IC}(\mathbb{I})=\mathbb{J}$.

Proof.

(1) It is straightforward to see that $\mathbb{I}^{\prime}$ is an idealistic filtration, and that any idealistic filtration containing $T$ necessarily contains $\mathbb{I}^{\prime}$. Therefore, $\mathbb{I}^{\prime}=\mathbb{I}$ by the definition of $\mathbb{I}=G(T)$.

(2) Let $\mathbb{I}^{\prime}$ be a $\mathfrak{D}$-saturated idealistic filtration containing $T$, or equivalently containing $\mathbb{I}$. Then it is clear that $\mathbb{I}^{\prime} \supset G\left(T^{\prime}\right)$. Therefore, in order 
to see $\mathfrak{D}(\mathbb{I})=G\left(T^{\prime}\right)$, we have only to show $G\left(T^{\prime}\right)$ is $\mathfrak{D}$-saturated, which follows from the fact that Diff $R_{R}^{t}$ is generated by $\left\{\partial_{X^{J}} ;|J| \leq t\right\}$ as an $R$-module, and the generalized product rule (cf. Lemma 1.2.1.2). The proof for the case

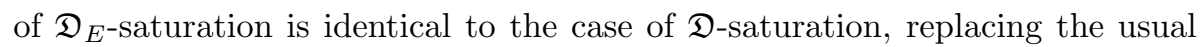
differentials with the logarithmic ones.

(3) Let $\mathbb{I}^{\prime}$ be an $\mathfrak{R}$-saturated idealistic filtration containing $\mathbb{I}$. As $\mathbb{I}^{\prime}$ satisfies condition (radical), we have $\mathbb{K} \subset \mathbb{I}^{\prime}$. Therefore, we conclude

$$
\overline{\mathbb{K}}_{a}=\bigcap_{b<a} \mathbb{K}_{a} \subset \bigcap_{b<a} \mathbb{I}_{b}^{\prime}=\mathbb{I}_{a}^{\prime},
$$

where the last equality follows since $\mathbb{I}^{\prime}$ satisfies condition (continuity) (cf. Remark 2.1.3.2(2)). That is to say, $\overline{\mathbb{K}} \subset \mathbb{I}^{\prime}$.

Thus, in order to see $\mathfrak{R}(\mathbb{I})=\overline{\mathbb{K}}$, we have only to show that $\overline{\mathbb{K}}$ itself is an idealistic filtration containing $\mathbb{I}$, satisfying conditions (radical) and (continuity).

First we show that $\overline{\mathbb{K}}$ is an idealistic filtration. We have only to check that $\overline{\mathbb{K}}_{a}(a>0)$ is closed under addition (cf. Definition 2.1.1.1 (1) and Remark 2.1.1.2 (1)), while the other conditions follow easily. Take $f, g \in \overline{\mathbb{K}}_{a}$. Then for any $b<a$, there exists $n \in \mathbb{Z}_{>0}$ such that $f^{n}, g^{n} \in \mathbb{I}_{n b}$. Then for any $k \in \mathbb{Z}_{>0}$, we have

$$
(f+g)^{k}=\sum_{i=0}^{k}\left(\begin{array}{c}
k \\
i
\end{array}\right) f^{i} g^{k-i} \in \mathbb{I}_{n b}^{\left\lfloor\frac{i}{n}\right\rfloor+\left\lfloor\frac{k-i}{n}\right\rfloor} \subset \mathbb{I}_{b(k-2 n)}
$$

since $\left\lfloor\frac{i}{n}\right\rfloor+\left\lfloor\frac{k-i}{n}\right\rfloor \geq \frac{k}{n}-2$. Therefore, we have $f+g \in \mathbb{K}_{b\left(1-2 n k^{-1}\right)}$. Since $b<a$ and $k>0$ are arbitrary (while $n$ depends only on $b$ ), we conclude $f+g \in \mathbb{K}_{c}$ for any $c<a$. Therefore, we have $f+g \in \bigcap_{c<a} \mathbb{K}_{c}=\overline{\mathbb{K}}_{a}$.

Secondly we check condition (continuity) for $\overline{\mathbb{K}}$. In fact, we have

$$
\overline{\mathbb{K}}_{a}=\bigcap_{b<a} \mathbb{K}_{b}=\bigcap_{b<a} \bigcap_{c<b} \mathbb{K}_{c}=\bigcap_{b<a} \overline{\mathbb{K}}_{b} .
$$

Therefore, $\overline{\mathbb{K}}$ satisfies condition (continuity) (cf. Remark 2.1.3.2 (2)).

Finally we check condition (radical) for $\overline{\mathbb{K}}$. Suppose $f^{n} \in \overline{\mathbb{K}}_{a}$. Fix $b<a$. Then $f^{n} \in \mathbb{K}_{b}$ by definition of $\overline{\mathbb{K}}$, and there exists $m \in \mathbb{Z}_{>0}$ such that $\left(f^{n}\right)^{m} \in$ $\mathbb{I}_{m b}$ by definition of $\mathbb{K}$. Therefore, we have $f \in \mathbb{K}_{n^{-1} b}$. Since $b<a$ is arbitrary, we have $f \in \bigcap_{b<a} \mathbb{K}_{n^{-1} b}=\overline{\mathbb{K}}_{n^{-1} a}$. Therefore, $\overline{\mathbb{K}}$ satisfies condition (radical).

(4) It is clear that, if $\mathbb{I}^{\prime}$ is an idealistic filtration containing $\mathbb{I}$ and satisfying (ic), then $\mathbb{J} \subset \mathbb{I}^{\prime}$. Thus, in order to see $\operatorname{IC}(\mathbb{I})=\mathbb{J}$, we have only to show that $\mathbb{J}$ itself is an idealistic filtration containing $\mathbb{I}$, satisfying condition (ic).

It is clear that $\mathbb{J}$ contains $\mathbb{I}$. Consider the graded subring $\mathfrak{G} \mathfrak{r}(\mathbb{I}):=\bigoplus_{a \in \mathbb{R}}$ $\times \mathbb{I}_{a} X^{a} \subset \bigoplus_{a \in \mathbb{R}} R X^{a}$, where $X$ is a variable transcendental over $R$, and where 
the structure of the graded $R$-algebra is given through multiplication rule $X^{a} X^{b}=X^{a+b}$.

Observe (cf. [Mat86]) that

$(f, a)$ is integral over $\mathbb{I} \Longleftrightarrow \mathfrak{G r}(\mathbb{I})\left[f X^{a}\right]$ is a finite $\mathfrak{G} \mathfrak{r}(\mathbb{I})$-module.

Observe also that

$$
\mathfrak{G} \mathfrak{r}(G(\mathbb{I},(f, a)))=\mathfrak{G} \mathfrak{r}(\mathbb{I})\left[f X^{a}\right] .
$$

Now from these observations it follows easily that $\mathbb{J}$ is an idealistic filtration, and that $\mathbb{I}$ satisfies condition (ic).

This completes the proof for Lemma 2.2.1.2.

\subsection{2. $\Re$-saturated implies integrally closed.}

Proposition 2.2.2.1. Let $\mathbb{I} \subset R \times \mathbb{R}$ be an idealistic filtration. If $\mathbb{I}$ is $\mathfrak{R}$-saturated, then $\mathbb{I}$ is integrally closed.

Proof. Let $\mathbb{I}$ be an $\mathfrak{R}$-saturated idealistic filtration. Suppose $(f, a) \in$ $R \times \mathbb{R}$ is integral over $\mathbb{I}$, i.e., $f$ satisfies a monic equation of the form

$(\star) \quad f^{n}+c_{1} f^{n-1}+\cdots+c_{n}=0$ with $\left(c_{i}, i a\right) \in \mathbb{I}$ for $i=1, \ldots, n$.

We want to show $(f, a) \in \mathbb{I}$.

If $a \leq 0$, then obviously $(f, a) \in \mathbb{I}$ (cf. conditions (o), (iii) in Remark 2.1.1.2 (1)). Thus, we may further assume $a>0$. Let

$$
\beta_{l}=1-\left(\frac{n-1}{n}\right)^{l} \quad\left(l \in \mathbb{Z}_{\geq 0}\right) .
$$

We show by induction that

$$
(\nabla)_{l} \quad\left(f, a \beta_{l}\right) \in \mathbb{I} .
$$

$(\nabla)_{0}$ is clear. Suppose we have shown $(\nabla)_{l}$. Using the monic equation $(\star)$, we have

$$
f^{n}=-\left(c_{1} f^{n-1}+\cdots+c_{n}\right)
$$

with

$$
\left(c_{i} f^{n-i}, a\left\{i+(n-i) \beta_{l}\right\}\right) \in \mathbb{I} \quad(1 \leq i \leq n) .
$$

Since $0 \leq \beta_{l}<1$, we have

$$
\min _{i}\left\{i+(n-i) \beta_{l}\right\}=1+(n-1) \beta_{l}=n \beta_{l+1} .
$$


Therefore, we conclude

$$
\left(f^{n}, a n \beta_{l+1}\right) \in \mathbb{I} .
$$

Since $\mathbb{I}$ is $\mathfrak{R}$-saturated, it follows from condition (radical)

$$
(\mathcal{Q})_{l+1} \quad\left(f, a \beta_{l+1}\right) \in \mathbb{I} .
$$

Thus $(\varnothing)_{l}$ is valid for all $l \in \mathbb{Z}_{\geq 0}$.

Note that $\lim _{l \rightarrow \infty} a \beta_{l}=a$. Therefore, by condition (continuity) satisfied by $\mathbb{I}$, we conclude

$$
(f, a) \in \mathbb{I} .
$$

Therefore, $\mathbb{I}$ is integrally closed.

This completes the proof of Proposition 2.2.2.1.

2.2.3. Analysis of interaction between $\mathfrak{D}$-saturation and $\mathfrak{R}$-saturation. So far, we have studied the $\mathfrak{D}$-saturation and $\mathfrak{R}$-saturation separately. In this subsection, we analyze the interaction of the operations of taking $\mathfrak{D}$-saturation and $\mathfrak{R}$-saturation. Under the assumption that $R$ has a regular system of parameters, our result is stated in the following proposition, which leads to the explicit construction of the $\mathfrak{B}$-saturation. Furthermore, the assumption is later removed for an idealistic filtration of r.f.g. type (cf. Corollary 2.4 .2 .3$)$.

Proposition 2.2.3.1. $\quad$ Let $\mathbb{I}$ be an idealistic filtration over $R$ which has a regular system of parameters $\left(x_{1}, \ldots, x_{d}\right)$. Then $\mathfrak{D} \mathfrak{R}(\mathbb{I}) \subset \mathfrak{R D}(\mathbb{I})$.

If $E$ is a simple normal crossing divisor defined by $\left\{x_{1} \cdots x_{m}=0\right\}$ for some $1 \leq m \leq d$, then $\mathfrak{D}_{E} \mathfrak{R}(\mathbb{I}) \subset \mathfrak{R} \mathfrak{D}_{E}(\mathbb{I})$.

Proof. We present a proof of the latter assertion in the logarithmic case, as the former is a special case of the latter $(E=\emptyset)$.

Step 1. Reduction of the assertion to the statement (\$).

By replacing $\mathbb{I}$ with $\mathfrak{D}_{E}(\mathbb{I})$ and via the obvious inclusion $\mathfrak{D}_{E} \mathfrak{R}(\mathbb{I}) \subset \mathfrak{D}_{E} \mathfrak{R}$ $\times\left(\mathfrak{D}_{E}(\mathbb{I})\right)$, we see that it suffices to prove the inclusion

$$
\mathfrak{D}_{E} \mathfrak{R}(\mathbb{I}) \subset \mathfrak{R}(\mathbb{I}),
$$

assuming $\mathbb{I}$ is $\mathfrak{D}_{E}$-saturated. In order to show the first inclusion above, by Lemma 2.2.1.2 (2), we have only to show

$$
\bigcup_{J}\left\{\left(D_{J}(f), a-|J|\right) ;(f, a) \in \mathfrak{R}(\mathbb{I})\right\} \subset \mathfrak{R}(\mathbb{I}),
$$


where $D_{J}=X^{J_{E}} \partial_{X^{J}}$. Now since $D_{J}=D_{j_{1} \mathbf{e}_{1}} \cdots D_{j_{d} \mathbf{e}_{d}}$, this second inclusion then follows if we can show, for $1 \leq i \leq d$, the inclusion below

$$
\bigcup_{j \geq 0}\left\{\left(D_{j \mathbf{e}_{i}}(f), a-j\right) ;(f, a) \in \mathfrak{R}(\mathbb{I})\right\} \subset \mathfrak{R}(\mathbb{I}) .
$$

Let $\mathbb{K}, \overline{\mathbb{K}} \subset R \times \mathbb{R}$ be as in Lemma 2.2.1.2 (3). We claim that we may even replace the range $\mathfrak{R}(\mathbb{I})$ of $(f, a)$ in the left hand side of the third inclusion with $\mathbb{K}$. That is to say, we claim it suffices to show

$$
(\diamond) \bigcup_{j \geq 0}\left\{\left(D_{j \mathbf{e}_{i}}(f), a-j\right) ;(f, a) \in \mathbb{K}\right\} \subset \mathfrak{R}(\mathbb{I}) .
$$

In fact, $(\diamond)$ implies

$$
\left\{\left(D_{j \mathbf{e}_{i}}(f), a-j\right) ;(f, a) \in \overline{\mathbb{K}}\right\} \subset \overline{\left\{\left(D_{j \mathbf{e}_{i}}(f), a-j\right) ;(f, a) \in \mathbb{K}\right\}} \subset \overline{\Re(\mathbb{I})},
$$

where, given a subset $T \subset R \times \mathbb{R}$, the subset $\bar{T}$ is defined by $\bar{T}_{a}=\bigcap_{b<a} T_{b}$. Since $\overline{\mathbb{K}}=\mathfrak{R}(\mathbb{I})=\overline{\mathfrak{R}(\mathbb{I})}$, this inclusion then would imply the third one.

Finally, we reduce $(\diamond)$ to the following general statement:

(a) $D_{j \mathbf{e}_{i}}(f) \in \mathfrak{R D} \mathfrak{D}_{E}\left(G\left\{\left(f^{n}, n a\right)\right\}\right)_{a-j} \quad(f \in R, a \in \mathbb{R}, 1 \leq i \leq d, n>0, j \geq 0)$.

Indeed, given $f \in \mathbb{K}_{a}$, there exists $n \in \mathbb{Z}_{>0}$ such that $f^{n} \in \mathbb{I}_{n a}$. Thus,

implies $D_{j \mathbf{e}_{i}}(f) \in \mathfrak{R D} \mathfrak{D}_{E}\left(G\left\{\left(f^{n}, n a\right)\right\}\right)_{a-j} \subset \mathfrak{R D} \mathfrak{D}_{E}(\mathbb{I})_{a-j}=\mathfrak{R}(\mathbb{I})_{a-j}$ since $\mathbb{I}$ is

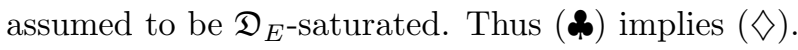

Therefore, we conclude that the assertion of the lemma is reduced to the statement (⿻).

Step 2. Setup for the inductional proof of $(\boldsymbol{\beta})$.

We fix $1 \leq i \leq d$, and omit $i$ from the notation in the following argument. For example, we denote $D_{j \mathbf{e}_{i}}$ by $D_{j}$. We also denote $\mathfrak{R \mathfrak { D } _ { E }}\left(G\left(\left\{\left(f^{n}, n a\right)\right\}\right)\right)$ by $\mathbb{J}$ to ease the notation.

Set $c=\lfloor a\rfloor$. We prove the statement (\$) by induction on $c$. We may assume $0 \leq j \leq c$, since otherwise we have $a-j<0$ and $\mathbb{J}_{a-j}=R$, in which case (\$) clearly holds.

Case 1. $c=0$.

In this case, $j$ must be 0 , and we obviously have

$$
\left(D_{j}(f), a-j\right)=(f, a) \in \mathfrak{R}\left(G\left(\left\{\left(f^{n}, n a\right)\right\}\right)\right) \subset \mathbb{J} .
$$

Thus (\$) holds.

Case 2. $\quad c \geq 1$. 
In this case, we show (o) in Steps 3, 4, and 5 using the inductional hypothesis.

Observe that

$$
\left(D_{j}(f), a-j-1\right) \in \mathfrak{R D _ { E }}\left(G\left(\left\{\left(f^{n}, n a-n\right)\right\}\right)\right) \subset \mathbb{J}
$$

for $0 \leq j \leq c-1=\lfloor a-1\rfloor$, from the inductional hypothesis.

Step 3. Construction of a sequence $\left\{b_{u, j}\right\}$.

Our strategy for showing (

$$
b_{0, j}=1(1 \leq j \leq c-1), \quad b_{0, c}=a-c, \quad b_{u, 0}=0(u \geq 0),
$$

to construct the (double) sequence of numbers $b_{u, j}$ indexed by $0 \leq j \leq c$ and $u \in \mathbb{Z}_{\geq 0}$ satisfying the following conditions $(\mathbf{Q})$ and $(\nabla)$ :

$$
\text { (ه) } \quad\left(D_{j}(f), a-j-b_{u, j}\right) \in \mathbb{J}, \quad(\nabla) \quad \lim _{u \rightarrow \infty} b_{u, j}=0
$$

We construct the numbers $b_{u, j}$ inductively according to the lexicographical order on the double index $(u, j)$. Suppose we have already constructed all $b_{\alpha, \beta}$ with $(\alpha, \beta)<(u, j)$. Then, we define the number $b_{u, j}$ by the following formula

$$
n b_{u, j}=\max \left(\{0\} \cup\left\{\sum_{t_{l}<j} b_{u, t_{l}}+\sum_{j \leq t_{l} \leq c} b_{u-1, t_{l}}+\sum_{c<t_{l}}\left(a-t_{l}\right) ; T \in S_{n, j}^{*}\right\}\right)
$$

where $S_{n, j}=\left\{T \in \mathbb{Z}_{\geq 0}^{n} ;|T|=n j\right\}$ and $S_{n, j}^{*}=S_{n, j} \backslash\{(j, \ldots, j)\}$.

\section{Step 4. Verification of $(\boldsymbol{\Lambda})$.}

By the argument in Step 2, condition ( $)$ holds at the initial state, i.e., if $u=0$ or $j=0$. We proceed to check condition $(\boldsymbol{\beta})$ by induction on the pair $(u, j)$ in the lexicographical order. Using the logarithmic version of the generalized product rule (cf. Lemma 1.2.2.2 (2)) for $f^{n}$, we compute

$$
(\sharp) \quad D_{j}(f)^{n}=D_{n j}\left(f^{n}\right)-\sum_{T \in S_{n, j}^{*}} \prod_{l=1}^{n} D_{t_{l}}(f) .
$$

Take $T \in S_{n, j}^{*}$. Then, by inductional hypothesis, we have

$$
\left(\prod_{l=1}^{n} D_{t_{l}}(f), \sum_{t_{l}<j}\left(a-t_{l}-b_{t_{l}, u}\right)+\sum_{j \leq t_{l} \leq c}\left(a-t_{l}-b_{t_{l}, u-1}\right)\right) \in \mathbb{J} .
$$


By definition of $b_{u, j}$ and the fact $\sum_{l}\left(a-t_{l}\right)=n a-\sum_{l} t_{l}=n a-n j$, we have

$$
\begin{aligned}
& \sum_{t_{l}<j}\left(a-t_{l}-b_{t_{l}, u}\right)+\sum_{j \leq t_{l} \leq c}\left(a-t_{l}-b_{t_{l}, u-1}\right) \\
& =\sum_{t_{l} \leq c}\left(a-t_{l}\right)-\sum_{t_{l}<j} b_{t_{l}, u}-\sum_{j \leq t_{l} \leq c} b_{t_{l}, u-1} \\
& =n(a-j)-\left(\sum_{t_{l}<j} b_{t_{l}, u}+\sum_{j \leq t_{l} \leq c} b_{t_{l}, u-1}+\sum_{t_{l}>c}\left(a-t_{l}\right)\right) \geq n(a-j)-n b_{u, j} .
\end{aligned}
$$

On the other hand, by definition of $\mathbb{J}$, we have $D_{n j}\left(f^{n}\right) \in \mathbb{J}_{n a-n j}$. Since $b_{u, j} \geq 0$ by definition, we have $\left(D_{n j}\left(f^{n}\right), n(a-j)-n b_{u, j}\right) \in \mathbb{J}$. Therefore, by virtue of the formula $(\sharp)$. we have $\left(D_{j}(f)^{n}, n(a-j)-n b_{u, j}\right) \in \mathbb{J}$. As $\mathbb{J}$ is $\mathfrak{R}$-saturated, we have $\left(D_{j}(f), a-j-b_{u, j}\right) \in \mathbb{J}$. Thus $(\boldsymbol{\$})$ holds for $(u, j)$, as desired.

Step 5. Verification of $(\nabla)$.

We have only to show the following inequality:

$$
\text { (b) } b_{u, j} \leq\left(1-n^{-j}\right)\left(1-n^{-c}\right)^{u-1} \quad(u \geq 1, j \geq 0) .
$$

In fact, since $b_{u, j} \geq 0$ by definition and $0<1-n^{-m}<1$, condition $(\odot)$ obviously follows from inequality (b).

We prove $(b)$ by induction on the pair $(u, j)$ in the lexicographical order.

Since $b_{u, 0}=0$, inequality (b) is valid for $j=0$. estimate

By definition of $b_{u, j}$ and from the fact $\sum_{c<t_{l}}\left(a-t_{l}\right)<0$, we have an

$$
n b_{u, j} \leq \max \left\{\sum_{t_{l}<j} b_{u, t_{l}}+\sum_{j \leq t_{l} \leq c} b_{u-1, t_{l}} ; T \in S_{n, j}^{*}\right\} .
$$

By inductional hypothesis, we observe the following (i) and (ii):

(i) For $t_{l}<j$, we have

$$
b_{u, t_{l}} \leq\left(1-n^{-t_{l}}\right)\left(1-n^{-c}\right)^{u-1} \leq\left(1-n^{1-j}\right)\left(1-n^{-c}\right)^{u-1}
$$

(ii) For $j \leq t_{l} \leq c$, we have

$$
b_{u-1, t_{l}} \leq\left(1-n^{-t_{l}}\right)\left(1-n^{-c}\right)^{u-2} \leq\left(1-n^{-c}\right)^{u-1} .
$$

We also mention that, for any $T=\left(t_{1}, \ldots, t_{n}\right) \in S_{n, j}^{*}$, there exists at least one $1 \leq l \leq n$ such that $t_{l}<j$.

By these observations, we obtain the following estimate:

$$
\begin{aligned}
n b_{u, j} & \leq\left(1-n^{1-j}\right)\left(1-n^{-c}\right)^{u-1}+(n-1)\left(1-n^{-c}\right)^{u-1} \\
& =\left(n-n^{1-j}\right)\left(1-n^{-c}\right)^{u-1}=n\left(1-n^{-j}\right)\left(1-n^{-c}\right)^{u-1}
\end{aligned}
$$


which implies inequality $(b)$ for $(u, j)$. This completes the proof for inequality $(b)$, and hence the verification of $(\nabla)$.

Step 6. Finishing argument.

In the previous Steps, we confirmed conditions $(\mathbf{Q})$ and $(\boldsymbol{Q})$. Consequently, since $\mathbb{J}$ is $\mathfrak{R}$-saturated, we have $D_{j}(f) \in \mathbb{J}_{a-j}$ for $0 \leq j \leq c$. Namely (()) holds for $c=\lfloor a\rfloor$. This completes the inductional proof of (⿻) stated in Step 2. This completes the proof of Proposition 2.2.3.1.

Corollary 2.2.3.2. $\quad$ Let $\mathbb{I}$ be an idealistic filtration over $R$ which has a regular system of parameters $\left(x_{1}, \ldots, x_{d}\right)$. Then $\mathfrak{B}(\mathbb{I})=\mathfrak{R D}(\mathbb{I})$.

If $E$ is a simple normal crossing divisor defined by $\left\{x_{1} \cdots x_{m}=0\right\}$ for some $1 \leq m \leq d$, then $\mathfrak{B}_{E}(\mathbb{I})=\mathfrak{R D}_{E}(\mathbb{I})$.

Proof. We present a proof of the latter assertion in the logarithmic case, as the former is a special case of the latter $(E=\emptyset)$.

Since $\mathfrak{B}_{E}(\mathbb{I})$ is $\mathfrak{D}_{E}$-saturated, we have $\mathfrak{B}_{E}(\mathbb{I}) \supset \mathfrak{D}_{E}(\mathbb{I})$. Then since $\mathfrak{B}_{E}(\mathbb{I})$ is $\mathfrak{R}$-saturated, we have $\mathfrak{B}_{E}(\mathbb{I}) \supset \mathfrak{R} \mathfrak{D}_{E}(\mathbb{I})$. In order to see the opposite inclusion, we have only to show that $\mathfrak{R D} E(\mathbb{I})$ is $\mathfrak{D}_{E}$-saturated. By Proposition 2.2.3.1, we see

$$
\mathfrak{R D _ { E }}(\mathbb{I}) \subset \mathfrak{D}_{E} \mathfrak{R \mathfrak { D } _ { E }}(\mathbb{I}) \subset \mathfrak{R \mathfrak { D } _ { E }} \mathfrak{D}_{E}(\mathbb{I})=\mathfrak{R \mathfrak { D } _ { E }}(\mathbb{I})
$$

Therefore, we conclude that $\mathfrak{R D} \mathfrak{D}_{E}(\mathbb{I})=\mathfrak{D}_{E} \mathfrak{R D} \mathfrak{D}_{E}(\mathbb{I})$ is $\mathfrak{D}_{E}$-saturated.

This completes the proof of Corollary 2.2.3.2.

Remark 2.2.3.3. Villamayor (cf. [Vil06a] [Vil06b] [EV07]) studies the interaction between $\mathfrak{D}$-saturation and $\mathfrak{R}$-saturation in the context of the Rees algebra (i.e., the relation between the differential structure and integral closure in his language), and obtains the results similar to ours independently. For example, Proposition 2.9 of [EV07] can be obtained if we apply our Proposition 2.2.3.1 to an idealistic filtration of r.f.g. type (cf. Corollary 2.4.2.3) and restrict our attention to its ideals at the integral levels. It seems, however, that the critical difference lies in the fact that our analysis involves the rational (and real) levels. The introduction of the rational levels is not a matter of theoretical convenience in our approach, but leads to a real difference in carrying out the steps of our algorithm, when we compute the invariant $\tilde{\mu}$ and construct the associated companion modification (cf. Remark 3.2.2.2 (6) and Parts II, III, IV). 


\section{§2.3. Idealistic filtration of r.f.g. type}

In Sections 2.1 and 2.2, we gave the definition of, and carried discussion on the properties of, an idealistic filtration in general. However, the idealistic filtrations we deal with in our algorithm are all of r.f.g. type (cf. Definition 2.1.1.1 (4)). Or rather to say, in order to guarantee for all the mechanisms and arguments in our algorithm to work properly, we need to know that the idealistic filtrations involved are all of r.f.g. type.

Since the operations of taking the $\mathfrak{D}$-saturation and $\mathfrak{R}$-saturation of a given idealistic filtration are essential in our algorithm, it is then a natural and important question whether the property of being of r.f.g. type is stable under these operations. The most important result of this section is to give an affirmative answer to this question: if an idealistic filtration $\mathbb{I}$ is of r.f.g. type, then so are $\mathfrak{D}(\mathbb{I})$ and $\mathfrak{R}(\mathbb{I})$. As a consequence, starting from an idealistic filtration of r.f.g. type, we see that we stay in the category of the idealistic filtrations of r.f.g. type throughout the execution of our algorithm. We remark that some related results can be found in [Hir03], discussing properties of an idealistic exponent.

For $\mathfrak{D}$-saturation, the verification of stability is elementary, using compatibility of $\mathfrak{D}$-saturation with localization (cf. Proposition 2.4.2.1 (2)) and using the explicit construction in Lemma 2.2.1.2.

For $\mathfrak{R}$-saturation, however, the verification of stability is rather subtle. Our argument presented here is due to Professor Shigefumi Mori, who showed us how the contents of [Nag57] can be adapted to verify the required stability under $\mathfrak{R}$-saturation. The essential point, starting from a given idealistic filtration of r.f.g. type $\mathbb{I}$, is to show the rationality and boundedness of the denominators of the numbers $a$ where $\mathfrak{R}(\mathbb{I})_{a}$ changes. Once the crucial rationality and boundedness are shown, stability can be reinterpreted as the finite generation of the integral closure as an $R$-algebra (in some finite extension of the field of fractions) of a certain graded ring, which is naturally associated to the idealistic filtration $\mathbb{I}$ of r.f.g. type.

We remark that, among the proposed approaches (cf. 0.5.2), the consideration of the rational levels and the actual use of the $\mathfrak{R}$-saturation in the execution of an algorithm are unique to the IFP, and hence so is its call for stability of r.f.g. type under $\mathfrak{R}$-saturation.

In this section, $R$ denotes the coordinate ring of an affine open subset of a variety $W$ smooth over $k$ of $\operatorname{char}(k)=p \geq 0$, or its localization by some multiplicative set. 
2.3.1. Stability of r.f.g. type under $\mathfrak{D}$-saturation. We show that the property of an idealistic filtration being of r.f.g. type is stable under $\mathfrak{D}$-saturation.

Proposition 2.3.1.1. Let $\mathbb{I} \subset R \times \mathbb{R}$ be an idealistic filtration. If $\mathbb{I}$ is of r.f.g. type, then so is its $\mathfrak{D}$-saturation $\mathfrak{D}(\mathbb{I})$ (or $\mathfrak{D}_{E}$-saturation $\mathfrak{D}_{E}(\mathbb{I})$ ).

Proof. Step 1. Reduction to the case where there exists a regular system of parameters $\left(x_{1}, \ldots, x_{d}\right)$ for $R$, where $d=\operatorname{dim} W$.

We take a finite affine cover $\left\{\operatorname{Spec} R_{g_{l}} ; g_{l} \in R\right\}_{l \in L}$ of $\operatorname{Spec} R$ with $\# L<\infty$ so that for each $R_{g_{l}}$ there exists a regular system of parameters for $R_{g_{l}}$.

Since $\mathbb{I}$ is of r.f.g. type, so is $\mathbb{I}_{g_{l}}$, its localization by $g_{l}$.

Suppose we have shown that $\mathfrak{D}\left(\mathbb{I}_{g_{l}}\right)$ is of r.f.g. type, i.e., there exists a finite set

$$
T_{\Lambda_{l}}=\left\{\left(f_{\lambda_{l}}, a_{\lambda_{l}}\right)\right\}_{\lambda_{l} \in \Lambda_{l}} \subset R_{g_{l}} \times \mathbb{Q}
$$

such that $\mathfrak{D}\left(\mathbb{I}_{g_{l}}\right)=G_{R_{g_{l}}}\left(T_{\Lambda_{l}}\right)$.

Observe that, since $\mathfrak{D}\left(\mathbb{I}_{g_{l}}\right)=\mathfrak{D}(\mathbb{I})_{g_{l}}$ by compatibility of localization with $\mathfrak{D}$-saturation (cf. Proposition 2.4.2.1 (2)), for each $\left(f_{\lambda_{l}}, a_{\lambda_{l}}\right)$, there exist $\left(h_{\lambda}, a_{\lambda}\right)$

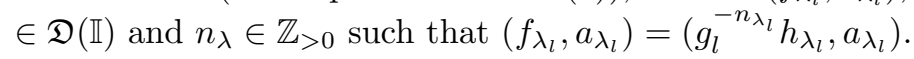

Then it is easy to see that the finite set

$$
T_{\Lambda}=\left\{\left(h_{\lambda_{l}}, a_{\lambda_{l}}\right) ; \lambda_{l} \in \Lambda_{l}, l \in L\right\} \subset \mathfrak{D}(\mathbb{I})
$$

generates $\mathfrak{D}(\mathbb{I})$, i.e., $\mathfrak{D}(\mathbb{I})=G_{R}\left(T_{\Lambda}\right)$. In fact, by construction, we have

$$
\mathfrak{D}(\mathbb{I})_{g_{l}} \supset G_{R}\left(T_{\Lambda}\right)_{g_{l}} \supset G_{R_{g_{l}}}\left(T_{\Lambda_{l}}\right)=\mathfrak{D}\left(\mathbb{I}_{g_{l}}\right)=\mathfrak{D}(\mathbb{I})_{g_{l}},
$$

i.e., $\mathfrak{D}(\mathbb{I})_{g_{l}}=G_{R}\left(T_{\Lambda}\right)_{g_{l}}$ for any $l \in L$, and hence $\mathfrak{D}(\mathbb{I})=G_{R}\left(T_{\Lambda}\right)$.

Step 2. Proof of the statement in the case where there exists a regular system of parameters $\left(x_{1}, \ldots, x_{d}\right)$ for $R$, where $d=\operatorname{dim} W$.

Take a finite set of generators $T_{\Lambda}$ of the form

$$
T_{\Lambda}=\left\{\left(f_{\lambda}, a_{\lambda}\right)\right\}_{\lambda \in \Lambda} \subset R \times \mathbb{Q}
$$

such that $\mathbb{I}=G\left(T_{\Lambda}\right)$. We may assume $a_{\lambda}>0 \quad \forall \lambda \in \Lambda$ by discarding those with $a_{\lambda} \leq 0$. Let

$$
T_{M}=\left\{\left(\partial_{X^{J}} f_{\lambda}, a_{\lambda}-|J|\right) ;\left(f_{\lambda}, a_{\lambda}\right) \in T_{\Lambda}, 0 \leq|J|<a_{\lambda}\right\} .
$$

Then clearly we have $\# T_{M}<\infty$ and $a_{\lambda}-|J| \in \mathbb{Q} \quad \forall \lambda$ and $\forall J$ with $0 \leq|J|<$ $a_{\lambda}$ 
Now it follows from Lemma 2.2.1.2 (2) that $\mathfrak{D}(\mathbb{I})=G\left(T_{M}\right)$. Therefore, we conclude that $\mathfrak{D}(\mathbb{I})$ is of r.f.g. type.

The proof for stability under $\mathfrak{D}_{E}$-saturation is identical. This completes the proof for Proposition 2.3.1.1.

2.3.2. Stability under $\mathfrak{R}$-saturation. We show that the property of an idealistic filtration being of r.f.g. type is stable under $\mathfrak{R}$-saturation. We deal with the problem of stability in terms of a certain graded ring which is naturally associated to an idealistic filtration $\mathbb{I}$ of r.f.g. type and which "describes" $\mathbb{I}$ in the sense stated below.

Definition 2.3.2.1. Let $A=\bigoplus_{n \in \mathbb{Z}_{\geq 0}} A_{q n} X^{q n} \subset \bigoplus_{n \in \mathbb{Z}_{\geq 0}} R X^{q n}=$ $R\left[X^{q}\right]$ be a graded $R$-subalgebra of the polynomial ring with one variable $X^{q}$ over $R$ for some $q \in \mathbb{Q}_{>0}$. Let $\mathbb{I} \subset R \times \mathbb{R}$ be an idealistic filtration. We say $A$ describes $\mathbb{I}$ if it satisfies the following condition:

$$
\mathbb{I}_{q a}=A_{q\lceil a\rceil} \text { for any } a \in \mathbb{R}_{\geq 0} .
$$

Lemma 2.3.2.2. Let $\mathbb{I} \subset R \times \mathbb{R}$ be an idealistic filtration. Then $\mathbb{I}$ is of r.f.g. type if and only if there exists $A$ which describes $\mathbb{I}$ (as stated in Definition 2.3.2.1) and which is finitely generated as an $R$-algebra.

Proof. Suppose that there exists such $A$ which describes $\mathbb{I}$ and which is generated by a finite set of homogeneous elements $\left\{f_{\lambda} X^{q n_{\lambda}}\right\}_{\lambda \in \Lambda}$ as a graded $R$-subalgebra in $R\left[X^{q}\right]$. Then $\mathbb{I}$ is generated by the finite set $\left\{\left(f_{\lambda}, q n_{\lambda}\right)\right\}_{\lambda \in \Lambda}$, and hence is of r.f.g. type.

Conversely, suppose that $\mathbb{I}$ is an idealistic filtration of r.f.g. type, generated by a finite set $T=\left\{\left(f_{\lambda}, \frac{n_{\lambda}}{\delta}\right)\right\}_{\lambda \in \Lambda} \subset R \times \mathbb{Q}$ for some $\delta \in \mathbb{Z}_{>0}$. It is immediate that, if we take the graded $R$-subalgebra $A$ of $R\left[X^{q}\right]$, with $q=\delta^{-1}$ and $A_{0}=R$, generated by the finite set $\left\{f_{\lambda} X^{\frac{i}{\delta}} ; \lambda \in \Lambda, 0 \leq i \leq n_{\lambda}\right\}$ over $R$, then $A$ describes I.

This completes the proof of Lemma 2.3.2.2.

We remark that if $\mathbb{I}$ is an idealistic filtration of r.f.g. type, and if $A \subset$ $R\left[X^{q}\right]$ is a graded $R$-subalgebra which describes $\mathbb{I}$ for some $q \in \mathbb{Q}_{>0}$, then $A$ is automatically finitely generated over $R$.

Corollary 2.3.2.3. $\quad$ Let $\mathbb{I}$ be an idealistic filtration of r.f.g. type. Then II satisfies condition (continuity). 
Proof. $\quad$ We want to show $\mathbb{I}_{a}=\bigcap_{b<a} \mathbb{I}_{b}$ for any $a \in \mathbb{R}$ (cf. Remark 2.1.3.2 $(2))$.

It is clear when $a \leq 0$ (cf. condition (o) in Definition 2.1.1.1 (2)).

Suppose $a>0$. By Lemma 2.3.2.2, there exists a graded $R$-subalgebra $A \subset R\left[X^{q}\right]$, for some $q \in \mathbb{Q}_{>0}$, which describes $\mathbb{I}$ and which is finitely generated as an $R$-algebra. Then by definition we have $\mathbb{I}_{q a}=A_{q\lceil a\rceil}$. Since $\mathbb{I}_{s} \supset \mathbb{I}_{t}$ for any $s<t$, we conclude

$$
\begin{aligned}
& \bigcap_{b<a} \mathbb{I}_{q b}=\bigcap_{\lceil a\rceil-1<b<a} \mathbb{I}_{q b}=\bigcap_{\lceil a\rceil-1<b<a} A_{q\lceil b\rceil}=\bigcap_{\lceil a\rceil-1<b<a} A_{q\lceil a\rceil}=A_{q\lceil a\rceil}=\mathbb{I}_{q a}, \\
& \text { i.e., } \mathbb{I}_{q a}=\bigcap_{b<a} \mathbb{I}_{q b} .
\end{aligned}
$$

This completes the proof of Corollary 2.3.2.3.

Proposition 2.3.2.4. Let $\mathbb{I} \subset R \times \mathbb{R}$ be an idealistic filtration. If $\mathbb{I}$ is of r.f.g. type, then so is its $\Re$-saturation.

Before beginning the proof of Proposition 2.3.2.4, we extract the essence that we need from Nagata's paper [Nag57] with some modifications.

Let $R$ be a noetherian domain, $K=Q(R)$ its field of fractions and $\mathfrak{a}=$ $\left(u_{1}, \ldots, u_{s}\right) \subset R$ a proper ideal of $R$ with a finite set of its generators $u_{j}$. Set $R_{j}=R\left[\frac{u_{1}}{u_{j}}, \ldots, \frac{u_{s}}{u_{j}}\right]$, and let $\overline{R_{j}}$ be its normalization in $Q\left(R_{j}\right)=K$ for each $j$. Let $\left\{P_{j k}\right\}_{k} \subset \operatorname{Spec} \overline{R_{j}}$ be the set of all the minimal primes of $u_{j} \overline{R_{j}}$. Note that it is a finite set and that, by Krull's Hauptidealsatz, the primes $P_{j k}$ are of height 1 . Let $R_{j k}=\left(\overline{R_{j}}\right)_{P_{j k}}$ be the localization of $\overline{R_{j}}$ at $P_{j k}$ for each $j, k$. Since $R_{j k}$ is a 1 -dimensional noetherian normal ring, it is a discrete valuation ring. We denote the valuation of $R_{j k}$ by $v_{j k}$ for each $j, k$. We consider the functions $\theta_{\mathfrak{a}}, \bar{\theta}_{\mathfrak{a}}: R \rightarrow \mathbb{R}_{\geq 0} \cup\{\infty\}$ defined by

$$
\begin{aligned}
& \theta_{\mathfrak{a}}(r)=\sup \left\{\frac{m}{n} ; r^{n} \in \mathfrak{a}^{m}, n, m \in \mathbb{Z}_{\geq 0}, n>0\right\}, \\
& \bar{\theta}_{\mathfrak{a}}(r)=\sup \left\{\frac{m}{n} ; r^{n} \in \overline{\mathfrak{a}^{m}}, n, m \in \mathbb{Z}_{\geq 0}, n>0\right\} .
\end{aligned}
$$

Using the notation as above, we have the following lemmas.

Lemma 2.3.2.5. For $n \in \mathbb{Z}_{>0}$, we have

$$
\overline{\mathfrak{a}^{n}}=R \cap \bigcap_{j, k} u_{j}^{n} R_{j k} .
$$


Proof. $\quad$ Firstly we show $\overline{\mathfrak{a}^{n}} \subset R \cap \bigcap_{j, k} u_{j}^{n} R_{j k}$. It suffices to show $\overline{\mathfrak{a}^{n}} \subset u_{j}^{n} R_{j k}$ for each $j, k$. Fix $j, k$ and take $f \in \overline{\mathfrak{a}^{n}}$. Then, there exists a monic equation

$$
f^{m}+a_{1} f^{m-1}+\cdots+a_{m}=0 \quad\left(a_{i} \in \mathfrak{a}^{i n}\right) .
$$

Considering the valuation $v_{j k}$ of this equation, there exists some $1 \leq i \leq m$ such that $v_{j k}\left(f^{m}\right)=v_{j k}\left(a_{i} f^{m-i}\right)$ and hence $v_{j k}\left(f^{i}\right)=v_{j k}\left(a_{i}\right)$. Since $\mathfrak{a} R_{j k}=u_{j}^{n} R_{j k}$, we have $v_{j k}\left(a_{i}\right) \geq i n \cdot v_{j k}\left(u_{j}\right)$. Consequently $v_{j k}(f) \geq n v_{j k}\left(u_{j}\right)$, and hence $f \in u_{j}^{n} R_{j k}$. Thus the inclusion $\overline{\mathfrak{a}^{n}} \subset u_{j}^{n} R_{j k}$ holds.

Secondly we show the opposite inclusion $\overline{\mathfrak{a}^{n}} \supset R \cap \bigcap_{j, k} u_{j}^{n} R_{j k}$.

Take $g \in R \cap \bigcap_{j, k} u_{j}^{n} R_{j k}$. Set $R^{\prime}=R\left[\frac{u_{1}^{n}}{g}, \ldots, \frac{u_{s}^{n}}{g}\right]$ and $\mathfrak{b}=\left(\frac{u_{1}^{n}}{g}, \ldots, \frac{u_{s}^{n}}{g}\right)$ $\subset R^{\prime}$. We show $g \in \overline{\mathfrak{a}}^{n}$ in the following Steps.

Step 1. We show $\mathfrak{b}=R^{\prime}$.

Assume $\mathfrak{b}$ is a proper ideal of $R^{\prime}$. Then there exists a valuation ring $\left(V, \mathfrak{m}_{V}\right)$ of $Q\left(R^{\prime}\right)=K$ such that $V \supset R^{\prime}$ and $\mathfrak{m}_{V} \cap R^{\prime} \supset \mathfrak{b}$. We denote its valuation as $v$. Take $j_{0}$ such that $v\left(u_{j_{0}}\right)=\min _{1 \leq i \leq s} v\left(u_{i}\right)$. Then, as $\frac{u_{i}}{u_{j_{0}}} \in V$ for each $i$, we have

$$
R_{j_{0}} \subset V \text { and hence } \overline{R_{j_{0}}} \subset V \text {. }
$$

Since $\frac{u_{j_{0}}^{n}}{g} \in \mathfrak{b} \subset \mathfrak{m}_{v}$, we have $g \notin u_{j_{0}}^{n} V$, and hence $g \notin u_{j_{0}}^{n} \overline{R_{j_{0}}}$. Now, since $\overline{R_{j_{0}}}$ is noetherian normal domain, principal ideal $u_{j_{0}}^{n} \overline{R_{j_{0}}}$ is represented as

$$
u_{j_{0}}^{n} \overline{R_{j_{0}}}=\overline{R_{j_{0}}} \cap \bigcap_{\mathrm{ht} \mathfrak{p}=1} \mathfrak{p}^{v_{\mathfrak{p}}\left(u_{j_{0}}^{n}\right)}\left(\overline{R_{j_{0}}}\right)_{\mathfrak{p}}=\overline{R_{j_{0}}} \cap \bigcap_{k} P_{j_{0} k}^{v_{j_{0} k}\left(u_{j_{0}}^{n}\right)} R_{j_{0} k} .
$$

Therefore there exists some $k$ such that

$$
g \notin P_{j_{0} k}^{v_{j_{0} k}\left(u_{j_{0}}^{n}\right)} R_{j_{0} k}=u_{j_{0}}^{n} R_{j_{0} k},
$$

which contradicts to the choice of $g$. Thus we have $\mathfrak{b}=R^{\prime}$.

Step 2. We show $g \in \overline{\mathfrak{a}^{n}}$.

Since $1 \in \mathfrak{b}$ by Step 1 , there exists $F\left(X_{1}, \ldots, X_{s}\right) \in R\left[X_{1}, \ldots, X_{s}\right]$ such that $F(0, \ldots, 0)=0$ and $F\left(\frac{u_{1}^{n}}{g}, \ldots, \frac{u_{s}^{n}}{g}\right)=1$. Setting $\operatorname{deg} F=n$, we obtain

$$
0=g^{n}\left\{1-F\left(\frac{u_{1}^{n}}{g}, \ldots, \frac{u_{s}^{n}}{g}\right)\right\}=g^{n}+c_{1} g^{n-1}+\cdots+c_{n} \text { with } c_{i} \in \mathfrak{a}^{i},
$$

a monic equation which shows $g \in \overline{\mathfrak{a}^{n}}$. This completes the proof of Lemma 2.3.2.5.

Lemma 2.3.2.6. Let $r \in R$. Then,

$$
\theta_{\mathfrak{a}}(r)=\bar{\theta}_{\mathfrak{a}}(r)=\min _{j, k}\left\{\frac{v_{j k}(r)}{v_{j k}\left(u_{j}\right)}\right\} \in \mathbb{Q} .
$$


Moreover, for $n, m \in \mathbb{Z}_{\geq 0}$ with $n>0, r^{n} \in \overline{\mathfrak{a}^{m}}$ if and only if $\frac{m}{n} \leq \bar{\theta}_{\mathfrak{a}}(r)$.

Proof. Step 1. We show the first equation $\theta_{\mathfrak{a}}(r)=\bar{\theta}_{\mathfrak{a}}(r)$.

Since $\mathfrak{a}^{m} \subset \overline{\mathfrak{a}^{m}}$, it is immediate that $\theta_{\mathfrak{a}}(r) \leq \bar{\theta}_{\mathfrak{a}}(r)$. We show $\theta_{\mathfrak{a}}(r) \geq \bar{\theta}_{\mathfrak{a}}(r)$. Take $n, m \in \mathbb{Z}_{\geq 0}$ with $n>0$ such that $r^{n} \in \overline{\mathfrak{a}^{m}}$. By definition, there exists a monic equation

$$
\left(r^{n}\right)^{c+1}+a_{1}\left(r^{n}\right)^{c}+\cdots+a_{c+1}=0 \quad \text { with } \quad a_{i} \in \mathfrak{a}^{i m} .
$$

We show

$$
\nabla_{t}: \quad r^{n(c+t)} \in \mathfrak{a}^{m t}\left(r^{n} R+\mathfrak{a}^{m}\right)^{c} \quad\left(t \in \mathbb{Z}_{>0}\right)
$$

by induction on $t$. Looking at the monic equation above, we have

$$
r^{n(c+1)} \in \mathfrak{a}^{m}\left(r^{n}\right)^{c}+\cdots+\mathfrak{a}^{(c+1) m} r^{n(c+t)}=\mathfrak{a}^{m}\left(r^{n} R+\mathfrak{a}^{m}\right)^{c},
$$

thus $\varnothing_{1}$ holds. For the case $t>1$, we have

$$
\begin{aligned}
r^{n(c+t)} & =r^{n} \cdot r^{n(c+t-1)} \in r^{n} \mathfrak{a}^{m(t-1)}\left(r^{n} R+\mathfrak{a}^{m}\right)^{c} \\
& \subset \mathfrak{a}^{m(t-1)}\left(r^{n(c+1)} R+\mathfrak{a}^{m}\left(r^{n} R+\mathfrak{a}^{m}\right)^{c}\right) \\
& \subset \mathfrak{a}^{m(t-1)}\left(\mathfrak{a}^{m}\left(r^{n} R+\mathfrak{a}^{m}\right)^{c}\right) \quad\left(\text { By } \varnothing_{1}\right) \\
& =\mathfrak{a}^{m t}\left(r^{n} R+\mathfrak{a}^{m}\right)^{c} .
\end{aligned}
$$

Thus $\varnothing_{t}$, and hence $r^{n(c+t)} \in \mathfrak{a}^{m t}$ holds for any $t \in \mathbb{Z}_{>0}$. It follows that

$$
\theta_{\mathfrak{a}}(r) \geq \sup \left\{\frac{m t}{n(c+t)} ; t \in \mathbb{Z}_{>0}\right\} \geq \frac{m}{n} .
$$

Since the numbers $n, m \in \mathbb{Z}_{\geq 0}$ with $n>0$ such that $r^{n} \in \overline{\mathfrak{a}^{m}}$ are taken arbitrarily, we have $\theta_{\mathfrak{a}}(r) \geq \bar{\theta}_{\mathfrak{a}}(r)$.

Step 2. We show the second equality.

By Lemma 2.3.2.5, we have

$$
\begin{aligned}
r^{n} \in \overline{\mathfrak{a}^{m}} & \Longleftrightarrow r^{n} \in u_{j}^{m} R_{j k} \quad(\forall j, k) \quad \Longleftrightarrow \quad v_{j k}\left(r^{n}\right) \geq v_{j k}\left(u_{j}^{m}\right) \\
& \Longleftrightarrow n v_{j k}(r) \geq m v_{j k}\left(u_{j}\right) \quad(\forall j, k) \\
& \Longleftrightarrow \frac{m}{n} \leq \min \left\{\frac{v_{j k}(r)}{v_{j k}\left(u_{j}\right)} ; j, k\right\}
\end{aligned}
$$

Therefore $\bar{\theta}_{\mathfrak{a}}(r)=\min \left\{\frac{v_{j k}(r)}{v_{j k}\left(u_{j}\right)} ; j, k\right\} \in \mathbb{Q}$. The "Moreover" part is now obvious.

This completes the proof of Lemma 2.3.2.6.

We now go back to the proof of Proposition 2.3.2.4. 
Proof of Proposition 2.3.2.4. Take a finite set $T=\left\{\left(f_{\lambda}, a_{\lambda}\right)\right\}_{\lambda \in \Lambda} \subset$ $R \times \mathbb{Q}$ such that $\mathbb{I}=G(T)$.

Step 1. We may assume $T \subset R \times\{L\}$ for some $L \in \mathbb{Z}_{>0}$. Replacing $T$ with $T \cap R \times \mathbb{R}_{>0}$, we may assume $T \subset R \times \mathbb{Q}_{>0}$. Set

$$
L=\min \left\{n \in \mathbb{Z}_{>0} ; \frac{n}{a_{\lambda}} \in \mathbb{Z}_{>0} \quad \forall \lambda \in \Lambda\right\} \quad \text { and } \quad T^{\prime}=\left\{\left(f_{\lambda}^{\frac{L}{a_{\lambda}}}, L\right)\right\}_{\lambda \in \Lambda}
$$

Then it is clear that $\mathfrak{R}(G(T))=\mathfrak{R}\left(G\left(T^{\prime}\right)\right)$. Therefore, by replacing $T$ with $T^{\prime}$, we may assume $T \subset R \times\{L\}$.

Step 2. Description of $\mathfrak{R}(\mathbb{I})$ in terms of the function $\theta_{I}$.

Let $I=\mathbb{I}_{L}$ be the ideal of the idealistic filtration $\mathbb{I}$ at level $L$. Define $\mathbb{J} \subset R \times \mathbb{R}$ by setting $\mathbb{J}_{L a}=\left\{f \in R ; \theta_{I}(f) \geq a\right\}$ for $a \in \mathbb{R}$. We show $\mathfrak{R}(\mathbb{I})=\mathbb{J}$. Since $\mathbb{I}=G(T)=G(I \times\{L\})$, we have $\mathbb{I}_{L a}=I^{\lceil a\rceil}$ for any $a \in \mathbb{R}$ by Lemma 2.2.1.2 (1). (We use the convention that $I^{-n}=R$ for $n \in \mathbb{Z}_{>0}$ ). Thus, by Lemma 2.2.1.2 (3), $\mathfrak{R}(\mathbb{I})=\overline{\mathbb{K}}$ where $\overline{\mathbb{K}} \subset R \times \mathbb{R}$ is defined by

$$
\overline{\mathbb{K}}_{L a}=\left\{f \in R ; \forall b<a, \exists n \in \mathbb{Z}_{>0} \text { s.t. } f^{n} \in \mathbb{I}_{n L b}=I^{\lceil n b\urcorner}\right\} \quad(a \in \mathbb{R}) .
$$

The condition above can be rephrased as follows:

$$
\begin{aligned}
& \left(\forall b<a, \exists n \in \mathbb{Z}_{>0} \text { s.t. } f^{n} \in I^{\lceil n b\rceil}\right) \\
& \Leftrightarrow\left(\sup \left\{b \in \mathbb{R}_{\geq 0} ; \exists n \in \mathbb{Z}_{>0} \text { s.t. } f^{n} \in I^{\lceil n b\rceil}\right\} \geq a\right) \\
& \Leftrightarrow\left(\sup \left\{\frac{\lceil n b\rceil}{n} ; \exists n \in \mathbb{Z}_{>0}, \exists b \in \mathbb{R}_{\geq 0} \text { s.t. } f^{n} \in I^{\lceil n b\rceil}\right\} \geq a\right) \\
& \Leftrightarrow\left(\sup \left\{\frac{m}{n} ; \exists n, m \in \mathbb{Z}_{>0} \text { with } n>0 \text { s.t. } f^{n} \in I^{m}\right\} \geq a\right) \\
& \Leftrightarrow \theta_{I}(f) \geq a .
\end{aligned}
$$

Thus $\mathfrak{R}(\mathbb{I})_{L a}=\overline{\mathbb{K}}_{L a}=\mathbb{J}_{L a}$ for $a \in \mathbb{R}$, hence $\mathfrak{R}(\mathbb{I})=\mathbb{J}$.

Step 3. There exists $\rho \in \mathbb{Z}_{>0}$ such that $\mathbb{J}_{a}=\mathbb{J}_{\lceil\rho\rceil] / \rho}$ for any $a \in \mathbb{R}$.

We apply Lemma 2.3.2.6 with $\mathfrak{a}=I$ to our setting. Let $\rho$ be a common multiple of $\left\{v_{j k}\left(u_{j}\right) ; j, k\right\}$. Take $f \in \mathbb{J}_{L a}$. Then, we have $\theta_{I}(f) \geq a$. Since $\rho \theta_{I}(f) \in \mathbb{Z}$ by Lemma 2.3.2.6, we have $\rho L \theta_{I}(f) \geq\lceil\rho L a\rceil$. Therefore, we have $f \in \mathbb{J}_{\lceil\rho L a\rceil / \rho}$, and hence $\mathbb{J}_{L a} \subset \mathbb{J}_{\lceil\rho(L a)\rceil / \rho}$. The opposite inclusion is clear by condition (iii) in Definition 2.1.1.1 for the idealistic filtration $\mathbb{J}$.

Step 4. We show $S_{1}$ describes $\mathfrak{R}(\mathbb{I})$ and $S_{1}={\overline{S_{0}}}^{R_{1}}$ in the following notation: Consider the graded $R$-algebras 


$$
\begin{array}{ll}
R_{0}=R\left[X^{L}\right] & \supset S_{0}=\bigoplus_{n \in \mathbb{Z}_{\geq 0}} I^{n} X^{L n} \\
R_{1}=R\left[X^{\frac{1}{\rho}}\right] & \supset \quad S_{1}=\bigoplus_{n \in \mathbb{Z}_{\geq 0}} \mathbb{J}_{\frac{n}{\rho}} X^{\frac{n}{\rho}}
\end{array}
$$

where $X$ is an indeterminate. We denote by ${\overline{S_{0}}}^{R_{1}}$ the integral closure of $S_{0}$ in $R_{1}$.

It is clear from Step 3 that $S_{1}$ describes $\mathbb{J}=\mathfrak{R}(\mathbb{I})$. We have only to prove $S_{1}={\overline{S_{0}}}^{R_{1}}$.

Firstly we show $S_{1} \subset{\overline{S_{0}}}^{R_{1}}$. Let $g X^{\frac{n}{\rho}} \in S_{1}$ be a homogeneous element of $S_{1}$. Since $g \in J_{\frac{n}{\rho}}$, we have $\bar{\theta}_{I}(g)=\theta_{I}(g) \geq \frac{n}{\rho L}$. Thus, by Lemma 2.3.2.6, we have $g^{\rho L} \in \overline{I^{n}}$. Therefore there exists a monic equation

$$
\left(g^{\rho L}\right)^{m}+c_{1}\left(g^{\rho L}\right)^{m-1}+\cdots+c_{m}=0 \text { with } c_{i} \in\left(I^{n}\right)^{i} .
$$

This in turn provides a monic equation of $g X^{\frac{n}{\rho}}$ over $S_{0}$, i.e.,

$$
\left(g X^{\frac{n}{\rho}}\right)^{\rho L m}+\left(c_{1} X^{L n}\right)\left(g X^{\frac{n}{\rho}}\right)^{\rho L(m-1)}+\cdots+c_{m} X^{L n m}=0 .
$$

Therefore, we have $S_{1} \subset{\overline{S_{0}}}^{R_{1}}$.

Secondly we show $S_{1} \supset \overline{S_{0}} R_{1}$. Take $g=\sum_{n \in \mathbb{Z}_{\geq 0}} g_{\frac{n}{\rho}} X^{\frac{n}{\rho}} \in{\overline{S_{0}}}^{R_{1}}$. Then we have a monic equation of $g$ over $S_{0}$, i.e.,

$$
\text { (•) } g^{m}+c_{1}\left(X^{L}\right) g^{m-1}+\cdots+c_{m}\left(X^{L}\right)=0 \quad \text { with } \quad c_{i}\left(X^{L}\right) \in S_{0} .
$$

Set $G=\sum_{n \in \mathbb{Z}_{\geq 0}} g_{\frac{n}{\rho}} X^{\frac{n}{\rho}} Y^{n} \in R_{1}[Y]$ where $Y$ is another indeterminate. By replacing $X$ by $X Y^{\rho}$ in $(\boldsymbol{A})$, we have a monic equation of $G$ over $S_{0}[Y]$, i.e., $G^{m}+c_{1}\left(X^{L} Y^{\rho L}\right) G^{m-1}+\cdots+c_{m}\left(X^{L} Y^{\rho L}\right)=0 \quad$ with $\quad c_{i}\left(X^{L} Y^{\rho L}\right) \in S_{0}[Y]$. Since ${\overline{S_{0}[Y]}}^{R_{1}[Y]}={\overline{S_{0}}}^{R_{1}}[Y]$ (cf. Alg. Comm., Chap. V, $\S 1, \mathrm{n}^{\mathrm{o}} 3$, Prop. 12 in [Bou64]), each coefficient of $Y^{n}$ in $G$ are integral over $S_{0}$, i.e.,

$$
g_{\frac{n}{\rho}} X^{\frac{n}{\rho}} \in{\overline{S_{0}}}^{R_{1}} \quad\left(n \in \mathbb{Z}_{\geq 0}\right) .
$$

Thus we may assume $g$ is a homogeneous element in $R_{1}$, say, $g=g_{\frac{l}{\rho}} X^{\frac{l}{\rho}}$. Looking at the coefficient of $X^{\frac{m l}{\rho}}$ in $(\boldsymbol{\uparrow})$, we have

$$
g_{\frac{l}{\rho}}^{m}+\alpha_{1} g_{\frac{l}{\rho}}^{m-1}+\cdots+\alpha_{m}=0
$$


where $\alpha_{n}$ is the coefficient of $X^{\frac{n l}{\rho}}$ in $c_{n} \in S_{0} \subset R_{1}$. Note that $\alpha_{n}=0$ if $n l \notin \rho L \mathbb{Z}$, and $\alpha_{n} \in I^{\frac{n l}{\rho L}}$ if $n l \in \rho L \mathbb{Z}$. Thus, for any $1 \leq n \leq m$, we have

$$
\alpha_{n} \in I^{\frac{n l}{\rho L}}=\mathbb{I}_{\frac{n l}{\rho}} \subset \mathfrak{R}(\mathbb{I})_{\frac{n l}{\rho}} .
$$

Since $\mathfrak{R}(\mathbb{I})$ is integrally closed by Proposition 2.2 .2 .1 , we have

$$
g_{\frac{l}{\rho}} \in \mathfrak{R}(\mathbb{I})_{\frac{l}{\rho}}=\mathbb{J}_{\frac{l}{\rho}} \quad \text { and hence } \quad g=g_{\frac{l}{\rho}} X^{\frac{l}{\rho}} \in \mathbb{J}_{\frac{l}{\rho}} X^{\frac{l}{\rho}} \subset S_{1} .
$$

Therefore, we have $S_{1} \supset{\overline{S_{0}}}^{R_{1}}$.

Step 5. We see that $S_{1}$ is finitely generated over $R$.

It is clear when $I=(0)$, since $S_{1}=R$. We assume $I \neq(0)$.

Since $R$ is normal, so is $R_{1}=R\left[X^{\frac{1}{\rho}}\right]$. Thus

$$
S_{1}={\overline{S_{0}}}^{R_{1}}={\overline{S_{0}}}^{Q\left(R_{1}\right)} .
$$

Note that $Q\left(R_{1}\right)$ is a finite extension of $Q\left(S_{0}\right)=Q\left(R\left[X^{L}\right]\right)$. By $\S 33$ of [Mat86], it follows that $S_{1}={\overline{S_{0}}}^{Q\left(R_{1}\right)}$ is a finite $S_{0}$-module. On the other hand, $S_{0}$ is finitely generated over $R$. Indeed, taking generators of $I$ as $I=\left(r_{1}, \ldots, r_{t}\right)$, we have $S_{0}=R\left[r_{1} X^{L}, \ldots, r_{t} X^{L}\right]$. Thus $S_{1}$ is also finitely generated over $R$.

Step 6. Finishing argument.

By Steps 2 and 3, we see that $S_{1}$ describes the idealistic filtration $\mathfrak{R}(\mathbb{I})$. Since $S_{1}$ is finitely generated over $R$, we conclude that $\mathfrak{R}(\mathbb{I})$ is r.f.g. type (cf. Lemma 2.3.2.2). This completes the proof of Proposition 2.3.2.4.

Corollary 2.3.2.7. $\quad$ Let $\mathbb{I} \subset R \times \mathbb{R}$ be an idealistic filtration. Assume $\mathbb{I}$ is of r.f.g. type. Then its $\mathfrak{R}$-saturation coincides with its integral closure, i.e.,

$$
\mathfrak{R}(\mathbb{I})=\mathrm{IC}(\mathbb{I}) .
$$

Proof. $\quad$ By Proposition 2.2.2.1, $\mathfrak{R}(\mathbb{I})$ is integrally closed.

Therefore, we have $\mathfrak{R}(\mathbb{I}) \supset \operatorname{IC}(\mathbb{I})$. Thus we have only to show $\mathfrak{R}(\mathbb{I}) \subset \operatorname{IC}(\mathbb{I})$.

By the same argument as in Step 1 of the proof of Proposition 2.3.2.4, we may assume that $\mathbb{I}$ is generated by a finite number of elements at level $L$. In fact, using the same notation, we see that $\mathfrak{R}(G(T))=\mathfrak{R}\left(G\left(T^{\prime}\right)\right)$ and $\operatorname{IC}(G(T))=\operatorname{IC}\left(G\left(T^{\prime}\right)\right)$. Then as shown in Step 4 of the proof of Proposition 2.3.2.4, the integral closure $S_{1} \subset R\left[X^{\frac{1}{\rho}}\right]$ of $S_{0}$ in $R\left[X^{\frac{1}{\rho}}\right]$ describes $\mathfrak{R}(\mathbb{I})$, while $S_{0}$ describes $\mathbb{I}$.

Take an element $(f, a) \in \mathfrak{R}(\mathbb{I})$. Then we have $\left(f, \frac{\lceil\rho a\rceil}{\rho}\right) \in \mathfrak{R}(\mathbb{I})$ (cf. Lemma 2.3.1.1), which implies $f X^{\frac{[\rho a]}{\rho}} \in S_{1}$. Now since $f X^{\frac{[\rho a]}{\rho}}$ is integral over $S_{0}$, by 
the same argument as in Step 4 of the proof of Proposition 2.3.2.4, we see that $\left(f, \frac{\lceil\rho a\rceil}{\rho}\right)$ is integral over $\mathbb{I}$, i.e., $\left(f, \frac{\lceil\rho a\rceil}{\rho}\right) \in \mathrm{IC}(\mathbb{I})$. Finally, since $a \leq \frac{\lceil\rho\rceil\rceil}{\rho}$, we conclude $(f, a) \in \mathrm{IC}(\mathbb{I})$. This shows the desired inclusion.

This completes the proof of Corollary 2.3.2.7.

\section{§2.4. Localization and completion of an idealistic filtration}

In this section, we discuss the notion of localization and completion of an idealistic filtration over $R$, associated to the localization and completion of $R$, respectively. Our main observation here is the compatibility of the operations of taking the generation, $\mathfrak{D}$-saturation, and $\mathfrak{R}$-saturation with localization and completion. The compatibility allows us to reduce the analysis of the global properties of these operations to the local or to the analytic ones, to which we may apply some explicit computations.

In this section $R$ denotes the coordinate ring of an affine open subset of a nonsingular variety $W$ over $k$.

\subsubsection{Definition.}

Definition 2.4.1.1. Let $\mathbb{I} \subset R \times \mathbb{R}$ be an idealistic filtration over $R$.

(1) (Localization) Let $S$ be a multiplicative set of $R$. Consider the subset $\mathbb{I}_{S} \subset R_{S} \times \mathbb{R}$ defined by

$$
\left(\mathbb{I}_{S}\right)_{a}=\left(\mathbb{I}_{a}\right)_{S}=\mathbb{I}_{a} \otimes_{R} R_{S} \quad(a \in \mathbb{R}) .
$$

Then $\mathbb{I}_{S}$ is an idealistic filtration, called the localization of $\mathbb{I}$ by $S$.

In case $P \in \operatorname{Spec} R$ is a point corresponding to a prime ideal $P \subset R$ (we use the same symbol for the point and prime ideal by abuse of notation) with $S=R \backslash P$, we often denote $\mathbb{I}_{S}$ by $\mathbb{I}_{P}$.

(2) (Completion) Let $\widehat{R}$ be the completion of $R$ with respect to a maximal ideal $\mathfrak{m} \subset R$. Consider the subset $\widehat{\mathbb{I}} \subset \widehat{R} \times \mathbb{R}$ defined by

$$
(\widehat{\mathbb{I}})_{a}=\widehat{\mathbb{I}_{a}}=\mathbb{I}_{a} \otimes_{R} \widehat{R} \quad(a \in \mathbb{R}) .
$$

Then $\widehat{\mathbb{I}}$ is an idealistic filtration, called the completion of $\mathbb{I}$ (with respect to $\mathfrak{m}$-adic topology).

Remark 2.4.1.2. We remark that, for idealistic filtrations $\mathbb{I}, \mathbb{I}^{\prime} \subset R \times \mathbb{R}$, the following conditions are equivalent: 
(1) $\mathbb{I} \subset \mathbb{I}^{\prime}$,

(2) $\mathbb{I}_{\mathfrak{m}} \subset \mathbb{I}_{\mathfrak{m}}^{\prime}$ for any maximal ideal $\mathfrak{m} \subset R$,

(3) $\widehat{\mathbb{I}} \subset \widehat{\mathbb{I}^{\prime}}$, where the completion "^" is taken with respect the m-adic topology, for any maximal ideal $\mathfrak{m} \subset R$.

In fact, fixing the level $a \in \mathbb{R}$, we see that the equivalence of the conditions on the idealistic filtrations follows from the equivalence of the corresponding conditions on the ideals, which is a standard result in commutative ring theory.

\subsubsection{Compatibility.}

\section{Proposition 2.4.2.1.}

(1) (Compatibility with generation) Let $T \subset R \times \mathbb{R}$ be a subset. Then we have

$$
G_{R}(T)_{S}=G_{R_{S}}(T), \quad \widehat{G_{R}(T)}=G_{\widehat{R}}(T) .
$$

In particular, if $\mathbb{I}=G(T)$ is of r.f.g. type, then so are $\mathbb{I}_{S}$ and $\widehat{\mathbb{I}}$.

(2) (Compatibility with $\mathfrak{D}$-saturation) Let $\mathbb{I} \subset R \times \mathbb{R}$ be an idealistic filtration. Then we have

$$
\mathfrak{D}(\mathbb{I})_{S}=\mathfrak{D}\left(\mathbb{I}_{S}\right), \quad \widehat{\mathfrak{D}(\mathbb{I})}=\mathfrak{D}(\widehat{\mathbb{I}}) .
$$

Let $E$ be a simple normal crossing divisor. Then we have

$$
\mathfrak{D}_{E}(\mathbb{I})_{S}=\mathfrak{D}_{E}\left(\mathbb{I}_{S}\right), \quad \widehat{\mathfrak{D}_{E}(\mathbb{I})}=\mathfrak{D}_{E}(\widehat{\mathbb{I}}) .
$$

(3) (Compatibility with $\mathfrak{R}$-saturation) Let $\mathbb{I} \subset R \times \mathbb{R}$ be an idealistic filtration of r.f.g. type. Then we have

$$
\mathfrak{R}(\mathbb{I})_{S}=\mathfrak{R}\left(\mathbb{I}_{S}\right), \quad \widehat{\mathfrak{R}(\mathbb{I})}=\mathfrak{R}(\widehat{\mathbb{I}}) .
$$

Proof.

(1) This follows easily from the explicit construction of the generation in Lemma 2.2.1.2 (1).

(2) We verify $\mathfrak{D}(\mathbb{I})_{S}=\mathfrak{D}\left(\mathbb{I}_{S}\right)$. Firstly we show the inclusion $\mathfrak{D}(\mathbb{I})_{S} \subset$ $\mathfrak{D}\left(\mathbb{I}_{S}\right)$. Note that $\mathfrak{D}\left(\mathbb{I}_{S}\right) \cap\{R \times \mathbb{R}\}$ is an idealistic filtration over $R$ containing $\mathbb{I}$, and being $\mathfrak{D}$-saturated by Lemma 1.1.2.1 (4). Therefore, we have

$$
\mathfrak{D}(\mathbb{I}) \subset \mathfrak{D}\left(\mathbb{I}_{S}\right) \cap\{R \times \mathbb{R}\} \subset \mathfrak{D}\left(\mathbb{I}_{S}\right) .
$$


At level $a \in \mathbb{R}$, this implies $\mathfrak{D}(\mathbb{I})_{a} \subset \mathfrak{D}\left(\mathbb{I}_{S}\right)_{a}$ and hence $\left(\mathfrak{D}(\mathbb{I})_{a}\right)_{S} \subset \mathfrak{D}\left(\mathbb{I}_{S}\right)_{a}$. That is to say, we have $\mathfrak{D}(\mathbb{I})_{S} \subset \mathfrak{D}\left(\mathbb{I}_{S}\right)$.

Secondly we show the opposite inclusion $\mathfrak{D}(\mathbb{I})_{S} \supset \mathfrak{D}\left(\mathbb{I}_{S}\right)$. Note that $\mathfrak{D}(\mathbb{I})_{S}$ is an idealistic filtration over $R_{S}$ containing $\mathbb{I}$, and hence containing $\mathbb{I}_{S}$. We claim that $\mathfrak{D}(\mathbb{I})_{S}$ is $\mathfrak{D}$-saturated. In fact, suppose $(f, a) \in \mathfrak{D}(\mathbb{I})_{S}$, i.e., $f \in$ $\left\{\mathfrak{D}(\mathbb{I})_{a}\right\}_{S}$. Then, for $d \in \operatorname{Diff}_{R_{S}}^{t}$, we see by Lemma 1.1.2.1 (7)

$$
d(f) \in \operatorname{Diff}_{R_{S}}^{t}\left(\left\{\mathfrak{D}(\mathbb{I})_{a}\right\}_{S}\right)=\left\{\operatorname{Diff}_{R}^{t}\left(\mathfrak{D}(\mathbb{I})_{a}\right)\right\}_{S} \subset\left\{\mathfrak{D}(\mathbb{I})_{a-t}\right\}_{S} .
$$

That is to say, we have $(d(f), a-t) \in \mathfrak{D}(\mathbb{I})_{S}$, checking condition (differential) for $\mathfrak{D}(\mathbb{I})_{S}$. Thus we have $\mathfrak{D}(\mathbb{I})_{S} \supset \mathfrak{D}\left(\mathbb{I}_{S}\right)$.

This completes the verification for $\mathfrak{D}(\mathbb{I})_{S}=\mathfrak{D}\left(\mathbb{I}_{S}\right)$.

The verification for $\widehat{\mathfrak{D}(\mathbb{I})}=\mathfrak{D}(\widehat{\mathbb{I}})$ is identical to the one above using again Lemma 1.1.2.1 (7), and left to the reader as an exercise.

The verification for the compatibility of localization and completion with $\mathfrak{D}_{E}$-saturation goes almost verbatim to the one above, replacing $\mathfrak{D}$ and Difft ${ }_{R}$ with $\mathfrak{D}_{E}$ and Diff ${ }_{R, E}^{t}$. We leave the verification of the statement of Lemma 1.1.2.1 (7) obtained by replacing $\operatorname{Diff}_{R}^{t}$ with $\operatorname{Diff}_{R, E}^{t}$ as an exercise to the reader, since it is identical to the one we gave in Chapter 1.

(3) We use the same notation and argument as in Step 1 through Step 4 of the proof of Proposition 2.3.2.4 (See also Remark 2.4.2.2 (1) below). First, since $\mathbb{I}, \mathbb{I}_{S}$, and $\widehat{\mathbb{I}}$ share the same set of generators $T$, we may take in Step 1 the common replacement $T^{\prime}$ at level $L$, which keeps the left hand side and right hand side of the equation for compatibility intact. Therefore, we may assume from the beginning that $\mathbb{I}$ is generated by $T \subset R \times\{L\}$. Let $I=\mathbb{I}_{L}$ and $A=\bigoplus_{n \in \mathbb{Z}_{>0}} I^{n} X^{L n} \subset R\left[X^{L}\right]$. Note that $A$ describes the idealistic filtration $\mathbb{I}$ (cf. Definition 2.3.2.1, Lemma 2.3.2.2). Moreover,

$$
A_{S}=\bigoplus_{n \in \mathbb{Z}_{\geq 0}} I_{S}^{n} X^{L n} \subset R_{S}\left[X^{L}\right] \text { and } \widehat{A}=\bigoplus_{n \in \mathbb{Z}_{\geq 0}} \widehat{I}^{n} X^{L n} \subset \widehat{R}\left[X^{L}\right]
$$

describe the localization $\mathbb{I}_{S}$ and completion $\widehat{\mathbb{I}}$, respectively.

Step 2 goes without any change for all $\mathbb{I}, \mathbb{I}_{S}$, and $\widehat{\mathbb{I}}$.

We take $\rho$ in Step 3 so that $\rho$ works for all $\mathbb{I}, \mathbb{I}_{S}$ and $\widehat{\mathbb{I}}$ simultaneously. Set $\bar{A}, \overline{A_{S}}, \overline{\widehat{A}}$ as the integral closures of $A$ in $R\left[X^{\frac{1}{\rho}}\right]$, of $A_{S}$ in $R_{S}\left[X^{\frac{1}{\rho}}\right]$, and of $\widehat{A}$ in $\widehat{R}\left[X^{\frac{1}{\rho}}\right]$, respectively. Then, in Step 4 , we see that $\bar{A}, \overline{A_{S}}, \overline{\widehat{A}}$ describe the idealistic filtrations $\mathfrak{R}(\mathbb{I}), \mathfrak{R}\left(\mathbb{I}_{S}\right), \mathfrak{R}(\widehat{\mathbb{I}})$, respectively.

On the other hand, since $\bar{A}$ describes $\mathfrak{R}(\mathbb{I})$, it follows by definition that $(\bar{A})_{S}$ and $\widehat{\bar{A}}$ describe the localization $\mathfrak{R}(\mathbb{I})_{S}$ and completion $\widehat{\mathfrak{R}(\mathbb{I})}$, respectively.

Now since the operation of taking the integral closure commutes with localization, we have $(\bar{A})_{S}=\overline{A_{S}}$. Thus we conclude $\mathfrak{R}(\mathbb{I})_{S}=\mathfrak{R}\left(\mathbb{I}_{S}\right)$. 
As to the question of commutativity of the operation of taking the integral closure with completion, recall that $R$ is a finitely generated $k$-algebra or its localization, hence that it is a Grothendieck ring. Since $\bar{A}$ is a finitely generated $R$-algebra by Step 5 of Proposition 2.3.2.4, $\bar{A}$ is also a Grothendieck ring. This allows us to conclude that $\widehat{\bar{A}}$ is normal, since $\bar{A}$ is also normal (See Remark 1 to Theorem 32. 6 in [Mat86]). Now $\widehat{\bar{A}}$ is integral over $\widehat{A}$, since $\bar{A}$ is integral over $A$. Therefore we conclude $\widehat{\bar{A}}=\widehat{\widehat{A}}$, and hence $\widehat{\mathfrak{R}(\mathbb{I})}=\mathfrak{R}(\widehat{\mathbb{I}})$.

This completes the proof of Proposition 2.4.2.1.

Remark 2.4.2.2.

(1) In Section 2.3, the base ring $R$ was assumed to be the coordinate ring of an affine open subset of a variety $W$ smooth over $k$, or its localization. We did not deal with the case where the base ring is the completion $\widehat{R}$. Note that the proof of Proposition 2.3.2.4 works just as well over the base ring being the completion $\widehat{R}$ from Step 1 through Step 4, but fails in Step 5, where $Q(\widehat{R}[X])$ is not finitely generated over $k$. Therefore, we do not claim the stability of the idealistic filtrations of r.f.g. type over $\widehat{R}$ under $\mathfrak{R}$-saturation.

Nevertheless, we should emphasize that the following assertion is valid:

If an idealistic filtration $\mathbb{I}$ over $R$ is of r.f.g. type, then so is $\mathfrak{R}(\widehat{\mathbb{I}})$.

Indeed, since $\mathfrak{R}(\mathbb{I})$ is of r.f.g. type by Proposition 2.3.2.4, the assertion is a direct consequence of compatibility $\mathfrak{R}(\widehat{\mathbb{I}})=\widehat{\mathfrak{R}(\mathbb{I})}$.

(2) The assumption of $\mathbb{I}$ being of r.f.g. type is indispensable in Proposition 2.4.2.1 (3). The following gives a counterexample to the assertion of compatibility with $\mathfrak{R}$-saturation when $\mathbb{I}$ is not of r.f.g. type: Let $\mathbb{I}=G(T)$ be an idealistic filtration over $R=k[x, y]$ where the set of generators $T$ is an infinite set given as below

$$
T=\left\{\left(\phi_{i} y, 1-i^{-1}\right) ; i \in \mathbb{Z}_{>0}\right\}, \phi_{i}=\prod_{j=1}^{i}(x-j) .
$$

We claim that, $\mathfrak{m}=(x, y)$ being the maximal ideal corresponding to the origin, we have

$$
\mathfrak{R}\left(\mathbb{I}_{\mathfrak{m}}\right)=G_{R_{\mathfrak{m}}}(\{(y, 1)\}),(y, 1) \notin \mathfrak{R}(\mathbb{I})_{\mathfrak{m}} .
$$

This implies that $\mathfrak{R}\left(\mathbb{I}_{\mathfrak{m}}\right) \neq \mathfrak{R}(\mathbb{I})_{\mathfrak{m}}$ and also that $\mathfrak{R}(\widehat{\mathbb{I}})=G_{\widehat{R}}(\{(y, 1)\}) \neq \widehat{\mathfrak{R}(\mathbb{I})} \not \ngtr$ $(y, 1)$ where the completion is taken with respect to $\mathfrak{m}$.

Since $\mathfrak{R}\left(\mathbb{I}_{\mathfrak{m}}\right)=G(\{(y, 1)\})$ is clear, we only show the second part of the claim $(y, 1) \notin \mathfrak{R}(\mathbb{I})_{\mathfrak{m}}$. Assume $(y, 1) \in \mathfrak{R}(\mathbb{I})_{\mathfrak{m}}$. Then there exists $f(x, y) \in k[x, y]$ such that $f(0,0) \neq 0$ and that $f y \in \mathfrak{R}(\mathbb{I})_{1}$. Let $\mathbb{K}$ be as in Lemma 2.2.1.2 (3). 
Then, for any $l \in \mathbb{Z}_{>0}$, we have $f y \in \mathbb{K}_{1-l^{-1}}$ and hence $f^{m l} y^{m l} \in \mathbb{I}_{m l-m}$ for some $m \in \mathbb{Z}_{>0}$. Since the generators in $T$ are homogeneous with respect to $y$, we see that $\mathbb{I}_{m l-m}$ is a homogeneous ideal with respect to $y$ (cf. Lemma 2.2.1.2 (1)). This implies $f(x, 0)^{m l} y^{m l} \in \mathbb{I}_{m l-m}$. By Lemma 2.2.1.2 (1), we then conclude

$$
f(x, 0)^{m l} \in\left(\phi_{i_{1}} \cdots \phi_{i_{r}} ; r \leq m l, r-\sum_{t=1}^{r} i_{t}^{-1} \geq m l-m\right) .
$$

Looking at the range $\left\{i_{1}, \ldots, i_{r}\right\}$, we have

$$
1-r^{-1} \sum_{t=1}^{r} i_{t}^{-1}=r^{-1}\left(r-\sum_{t=1}^{r} i_{t}^{-1}\right) \geq(m l)^{-1}(m l-m)=1-l^{-1},
$$

and hence

$$
l^{-1} \geq r^{-1} \sum_{t=1}^{r} i_{t}^{-1} \geq\left(\max _{t} i_{t}\right)^{-1} .
$$

This implies that each range $\left\{i_{1}, \ldots, i_{r}\right\}$ contains at least one $i_{t}$ with $i_{t} \geq$ l. Therefore, we have $\phi_{l} \mid f(x, 0)^{m l}$ and hence $\phi_{l} \mid f(x, 0)$. Since $l \in \mathbb{Z}_{>0}$ is arbitrary, we conclude $f(x, 0)=0$, contradicting the assumption $f(0,0) \neq 0$. This contradiction shows $(y, 1) \notin \mathfrak{R}(\mathbb{I})_{\mathfrak{m}}$.

We end this section by stating a corollary which says that the results of 2.2.3 hold for an idealistic filtration $\mathbb{I}$ over $R$ which is essentially of finite type over $k$, without assuming it has a regular system of parameters, if $\mathbb{I}$ is of r.f.g. type.

Corollary 2.4.2.3. $\quad$ Let $\mathbb{I}$ be an idealistic filtration of r.f.g. type over $R$ which is essentially of finite type over $k$. Then, we have

$$
\mathfrak{D} \mathfrak{R}(\mathbb{I}) \subset \mathfrak{R D}(\mathbb{I}), \quad \mathfrak{B}(\mathbb{I})=\mathfrak{R D}(\mathbb{I}) .
$$

Let $E$ be a simple normal crossing divisor. Then we have

$$
\mathfrak{D}_{E} \mathfrak{R}(\mathbb{I}) \subset \mathfrak{R D} \mathfrak{D}_{E}(\mathbb{I}), \quad \mathfrak{B}_{E}(\mathbb{I})=\mathfrak{R D} \mathfrak{D}_{E}(\mathbb{I}) .
$$

In particular, the operation of taking the $\mathfrak{B}$-saturation or $\mathfrak{B}_{E}$-saturation is compatible with localization or completion for an idealistic filtration of r.f.g. type, and the property of being r.f.g. type is stable under the operation.

Proof. Firstly we show the inclusion $\mathfrak{D} \mathfrak{R}(\mathbb{I}) \subset \mathfrak{R D}(\mathbb{I})$. By Proposition 2.4.2.1, it suffices to check the inclusion over the localization of $R$ at an arbitrary maximal ideal. Then, since the localization admits a regular system of 
parameters, we can apply Proposition 2.2.3.1 to verify the inclusion. Secondly, in order to prove $\mathfrak{B}(\mathbb{I})=\mathfrak{D} \mathfrak{R}(\mathbb{I})$, we can repeat the argument in Corollary 2.2.3.2, which is valid regardless whether $R$ has a regular system of parameters or not, once we have the inclusion.

The proof of the logarithmic case is almost identical to the one above.

\section{Chapter 3. Leading Generator System}

The purpose of this chapter is to analyze the leading terms of the elements (at the nonnegative integral levels) of an idealistic filtration (localized at a closed point), i.e., to analyze the structure of the graded ring formed by the lowest terms of their power series expansions. Even though our analysis is elementary, it leads to the most important notion in the Idealistic Filtration Program, i.e., that of a leading generator system. In this chapter, we only give the definition of a leading generator system. However, it could be said that a large portion of our entire series of papers, though written with resolution of singularities in mind as the principal goal, is a treatise on the properties of a leading generator system in its own light.

We should emphasize that the idea of analyzing the leading terms (of the elements in the defining ideal) for the study of a singularity is nothing new and as classical as the word "tangent cone". Even in a more specific context of the problem of resolution of singularities, Hironaka and Oda (cf. [Hir70] [Oda73] [Oda83] [Oda87]), among others, realized its importance early on and made an extensive analysis developing the theory of the so-called Hironaka's additive group scheme. We also refer the reader to Pomerol's paper [Pom74] on the subject, which leads to Giraud's attempt to find an appropriate notion of a hypersurface of maximal contact in positive characteristic (cf. [Gir74] [Gir75]).

It is only natural that the recent approaches toward resolution of singularities in positive characteristic (cf. 0.5.2) have found renewed interests in these classical ideas. Villamayor pays special attention to the "differential structure" of the Rees algebras and their leading terms, and studies Hironaka's $\tau$-invariant in his approach using "generic projection" (cf. [Vil06a] [Vil06b] [EV07]). Włodarczyk [Wło07] puts the notion of " $p$-order" at the center of his approach in pursuit of a hypersurface of maximal contact in positive characteristic, finding inspiration in Giraud's work. Our approach is no exception drawing its inspiration from the afore-mentioned works of the classical masters.

However, the IFP seems to stand out unique in its systematic use of the notion of a leading generator system as a collective substitute for the notion of a hypersurface of maximal contact (cf. 0.2.3), and we carry out the program to 
the extent that we can present an explicit algorithm in positive characteristic (in Part IV) having the algorithm in characteristic zero as its prototype, though the problem of termination still remains (cf. $\S 0.3$ ). While the discussion of how we actually make the systematic use of a leading generator system in our algorithm has to wait for Part III and Part IV, the role of this chapter establishing the foundation is no less important.

In the following, we give a more technical briefing of the notion of a leading generator system.

Our analysis in this chapter is local, or even analytically local. Accordingly, we consider an idealistic filtration $\mathbb{I}$ over $R$ where $R$ is taken to be the localization at a maximal ideal corresponding to a closed point $P \in W$ of the coordinate ring of an affine open subset of a variety $W$ smooth over an algebraically closed field $k$ of $\operatorname{char}(k)=p \geq 0$, or its completion. We denote by $\mathfrak{m}$ the maximal ideal of $R$.

It is worth emphasizing that the main results of this chapter are obtained assuming that the idealistic filtration is $\mathfrak{D}$-saturated.

The main object of our study is the graded $k$-subalgebra $L(\mathbb{I})=\bigoplus_{n \in \mathbb{Z}_{\geq 0}}$ $\times L(\mathbb{I})_{n}$, formed by the leading terms (of the ideals at the nonnegative integral levels of the idealistic filtration (cf. §3.1)), of the graded ring $G=$ $\bigoplus_{\geq 0} \mathfrak{m}^{n} / \mathfrak{m}^{n+1}=\bigoplus_{n \in \mathbb{Z}_{\geq 0}} G_{n}$, which is isomorphic to a polynomial ring with $d(=\operatorname{dim} W)$-variables over $k$.

In characteristic zero, if $\mathbb{I}$ is $\mathfrak{D}$-saturated, $L(\mathbb{I})$ is generated as a graded algebra over $k$ by its degree one component $L(\mathbb{I})_{1}$, i.e., $L(\mathbb{I})=k\left[L(\mathbb{I})_{1}\right]$. Moreover, the hypersurfaces of maximal contact correspond exactly to the elements of the form $(h, 1) \in \mathbb{I}$ whose leading terms belong to $L(\mathbb{I})_{1} \backslash\{0\}$, i.e., $\bar{h}=(h \bmod \mathfrak{m}) \in L(\mathbb{I})_{1} \backslash\{0\}$. A fundamental observation of the IFP is then that the invariants we need to build a sequence of blowups for resolution of singularities can be all constructed from a collection $\left\{\left(h_{i}, 1\right)\right\} \subset \mathbb{I}$ with $\left\{\overline{h_{i}}\right\}$ forming a basis of $L(\mathbb{I})_{1}$ and hence generating the graded algebra $L(\mathbb{I})$, instead of taking a hypersurface of maximal contact one by one.

In characteristic $\operatorname{char}(k)=p>0$, in contrast, $L(\mathbb{I})$ may not be generated as a graded algebra over $k$ by its degree one component $L(\mathbb{I})_{1}$ even if $\mathbb{I}$ is $\mathfrak{D}$ saturated. Or worse, $L(\mathbb{I})_{1}$ may be 0 , i.e., there is no hypersurface of maximal contact. However, if $\mathbb{I}$ is $\mathfrak{D}$-saturated, $L(\mathbb{I})$ is generated as a graded algebra over $k$ by $\bigcup_{e \in \mathbb{Z}_{\geq 0}} L(\mathbb{I})_{p^{e}}^{\text {pure }}$, i.e.,

$$
L(\mathbb{I})=k\left[\bigcup_{e \in \mathbb{Z}_{\geq 0}} L(\mathbb{I})_{p^{e}}^{\text {pure }}\right],
$$


where $L(\mathbb{I})_{p^{e}}^{\text {pure }} \subset L(\mathbb{I})_{p^{e}}$ is the subspace consisting of "pure" elements. (We call an element $w \in L(\mathbb{I})_{p^{e}}$ "pure" if $w=v^{p^{e}}$ for some $v \in G_{1}$.) Observing that there is a sequence of inclusions

$$
L(\mathbb{I})_{1}=L(\mathbb{I})_{p^{0}}^{\text {pure }}, \quad\left\{L(\mathbb{I})_{p^{0}}^{\text {pure }}\right\}^{p} \subset L(\mathbb{I})_{p^{1}}^{\text {pure }}, \quad\left\{L(\mathbb{I})_{p^{1}}^{\text {pure }}\right\}^{p} \subset L(\mathbb{I})_{p^{2}}^{\text {pure }} \cdots
$$

which stabilizes to a sequence of equalities after some point, i.e., there exists $e_{M} \in \mathbb{Z}_{\geq 0}$ such that for $e>e_{M}$ the above inclusions become equalities

$$
\left\{L(\mathbb{I})_{p^{e-1}}^{\text {pure }}\right\}^{p}=L(\mathbb{I})_{p^{e}}^{\text {pure }},
$$

we are led to the following notion of a leading generator system.

We call a subset $\mathbb{H}=\left\{\left(h_{i j}, p^{e_{i}}\right)\right\} \subset \mathbb{I}$ a leading generator system if it satisfies the following conditions:

(i) $h_{i j} \in \mathfrak{m}^{p^{e_{i}}}$ and $\overline{h_{i j}}=\left(h_{i j} \bmod \mathfrak{m}^{p^{e_{i}}+1}\right) \in L(\mathbb{I})_{p^{e_{i}}}^{\text {pure }}$ for any $i, j$,

(ii) $\left\{{\overline{h_{i j}}}^{p^{e-e_{i}}} ; e_{i} \leq e\right\}$ consists of $\#\left\{(i, j) ; e_{i} \leq e\right\}$-distinct elements, and forms a $k$-basis of $L(\mathbb{I})_{p^{e}}^{\text {pure }}$ for any $e \in \mathbb{Z}_{\geq 0}$.

Therefore, if $\mathbb{I}$ is $\mathfrak{D}$-saturated, the leading terms of the elements in the leading generator system generates $L(\mathbb{I})$ as a graded algebra over $k$, i.e.,

$$
L(\mathbb{I})=k\left[\left\{\overline{h_{i j}}\right\}\right] .
$$

(Note that we have $\operatorname{dim}_{k} L(\mathbb{I})_{p^{e}}^{\text {pure }} \leq \operatorname{dim} W$ for any $e \in \mathbb{Z}_{\geq 0}$ and hence that condition (ii) implies $\# \mathbb{H} \leq \operatorname{dim} W$.)

In the existing algorithms in characteristic zero the notion of a hypersurface of maximal contact is the key for their inductive structure on dimension to function via the invariant unit $(w, s)$ (cf. 0.2.1.3.). According to the IFP we try to carry out an algorithm, even in positive characteristic, where the notion of a leading generator system provides a new inductive structure via the invariant unit $(\sigma, \widetilde{\mu}, s)$, in which $\sigma$, roughly speaking, plays the role of dimension (cf. 0.2.3.2.).

\section{§3.1. Analysis of the leading terms of an idealistic filtration}

\subsubsection{Definitions.}

Definition 3.1.1.1.

(1) Let $\mathbb{I}$ be an idealistic filtration over $R$ with its maximal ideal $\mathfrak{m}$. Recall that the maximal ideal $\mathfrak{m}$ corresponds to a closed point $P \in W$. Set

$$
G=\bigoplus_{n \in \mathbb{Z}_{\geq 0}} \mathfrak{m}^{n} / \mathfrak{m}^{n+1}=\bigoplus_{n \in \mathbb{Z}_{\geq 0}} G_{n} .
$$


We define the graded $k$-subalgebra

$$
L(\mathbb{I})=\bigoplus_{n \in \mathbb{Z}_{\geq 0}} L(\mathbb{I})_{n} \subset G,
$$

which we call the leading algebra of the idealistic filtration $\mathbb{I}$ at $P$, by setting

$$
L(\mathbb{I})_{n}=\left\{\bar{f}=\left(f \bmod \mathfrak{m}^{n+1}\right) ;(f, n) \in \mathbb{I}, f \in \mathfrak{m}^{n}\right\} .
$$

Note that $L(\mathbb{I})_{0}=k$ by condition (o) in Definition 2.1.1.1 (2).

(2) Set $p=\operatorname{char}(k)$ when $k$ is of positive characteristic, or $p=\infty$ when $k$ is of characteristic zero. For $e \in \mathbb{Z}_{\geq 0}$ with $p^{e} \in \mathbb{Z}_{>0}$, we define the pure part $L(\mathbb{I})_{p^{e}}^{\text {pure }}$ of $L(\mathbb{I})_{p^{e}}$ by the formula

$$
L(\mathbb{I})_{p^{e}}^{\text {pure }}=L(\mathbb{I})_{p^{e}} \cap F^{e}\left(G_{1}\right) \subset L(\mathbb{I})_{p^{e}},
$$

where $F^{e}$ is the $e$-th power of the Frobenius map of $G$ (cf. Definition 1.3.1.1).

An element $w \in L(\mathbb{I})_{p^{e}}$ is called pure if $w \in L(\mathbb{I})_{p^{e}}^{\text {pure }}$.

Remark 3.1.1.2. If we choose a regular system of parameters $\left(x_{1}, \ldots, x_{d}\right)$ for $R$, there is a natural isomorphism $G \cong k\left[x_{1}, \ldots, x_{d}\right]$. Through this isomorphism, we may identify $G$ with the polynomial ring over $k$.

We use the convention that $\infty^{n}=\infty$ for $n \in \mathbb{Z}_{>0}$ and $\infty^{0}=1$ (cf. 0.2.3.2.1.). Therefore, the only pure part we consider in characteristic zero is in degree one, where we have

$$
L(\mathbb{I})_{\infty^{0}}^{\text {pure }}=L(\mathbb{I})_{1}^{\text {pure }}=L(\mathbb{I})_{1} .
$$

In other words, in characteristic zero, all the pure elements are in degree one.

We see that $L(\mathbb{I})_{n}$ is a $k$-vector subspace of $G_{n}$, which follows from the definition of an idealistic filtration $\mathbb{I}$. Using the assumption that $k$ is algebraically closed, we also see that $L(\mathbb{I})_{p^{e}}^{\text {pure }}$ is a $k$-vector subspace of $L(\mathbb{I})_{p^{e}}$.

3.1.2. Heart of our analysis. At the heart of our analysis sits the following lemma, whose origin can be traced back to Hironaka and Oda. (See, e.g., [Oda87] for the notion of the flag of Frobenius linear subspaces.) Since it is quite fundamental to the IFP, we present our own proof, despite its elementary nature, for the sake of completeness and for the purpose of fixing the notation for later use.

Lemma 3.1.2.1. Let $G=k\left[x_{1}, \ldots, x_{d}\right]$ be the polynomial ring over $k$ with $d$ variables $X=\left(x_{1}, \ldots, x_{d}\right)$. We regard $G$ as a graded $k$-algebra with 
natural grading defined by the degree. We define "p" as in Definition 3.1.1.1 and we use the same convention as in Remark 3.1.1.2.

Let $L=\bigoplus_{n \in \mathbb{Z}_{\geq 0}} L_{n} \subset G$ be a graded $k$-subalgebra of $G$ with $L_{0}=G_{0}=$ k. Suppose that $L$ is $\mathfrak{D}$-saturated in the sense that it satisfies the following condition:

$$
f \in L, \partial \in \operatorname{Diff}_{G} \Longrightarrow \partial(f) \in L .
$$

Then $L$ is generated as a graded algebra over $k$ by its pure part $L^{\text {pure }}:=$ $\bigsqcup_{e \in \mathbb{Z}_{\geq 0}} L_{p^{e}}^{\text {pure }}$ where $L_{p^{e}}^{\text {pure }}=L_{p^{e}} \cap F^{e}\left(G_{1}\right) \subset L_{p^{e}}$, i.e., $L=k\left[L^{\text {pure }}\right]$.

Moreover, we can choose $\left\{e_{1}<\cdots<e_{M}\right\} \subset \mathbb{Z}_{\geq 0}$ and $V_{1} \sqcup \cdots \sqcup V_{M} \subset G_{1}$ with $V_{i}=\left\{v_{i j}\right\}_{j}$ satisfying the following conditions:

(i) $F^{e_{i}}\left(V_{i}\right) \subset L_{p^{e_{i}}}^{\text {pure }}$ for $1 \leq i \leq M$,

(ii) $\bigsqcup_{e_{i} \leq e} F^{e}\left(V_{i}\right)$ is a $k$-basis of $L_{p^{e}}^{\text {pure }}$ for any $e \in \mathbb{Z}_{\geq 0}$.

In particular, we have $L=k\left[\bigsqcup_{i=1}^{M} F^{e_{i}}\left(V_{i}\right)\right]$ with $\sum_{i=1}^{M} \# V_{i} \leq d$.

Proof. We prove the following assertion

$$
\left(\text { () } d \quad L=k\left[L^{\text {pure }}\right]\right.
$$

by induction on the number of variables $d$. When $d=0$, we have $G=L=k$ and $L^{\text {pure }}=\emptyset$. Thus we obviously have $(\nabla)_{0}$.

Now we prove $(\nabla)_{d}$ assuming $(\nabla)_{d-1}$. Take $f \in L$. It suffices to show $f \in k\left[L^{\text {pure }}\right]$. We may assume that $f$ is homogeneous of degree $r \in \mathbb{Z}_{\geq 0}$, i.e., $f \in L_{r}$. Set

$$
s=\max \left\{t \in \mathbb{Z}_{\geq 0} ; f \in F^{t}(G)\right\}, \quad r=r^{\prime} p^{s},
$$

and take $g \in G_{r^{\prime}}$ such that $f=g^{p^{s}}$. We write $g=\sum_{|J|=r^{\prime}} c_{J} X^{J} \in G_{r^{\prime}}$ with $c_{J} \in k$.

By the maximality of $s$ (and since $k$ is algebraically closed), we observe that there exists $J_{o}$ with $\left|J_{o}\right|=r^{\prime}$ such that $c_{J_{o}} \neq 0$ and that $p \nmid J_{o}=\left(j_{o 1}, \ldots, j_{o d}\right)$, i.e., $p \nmid j_{\text {o } \alpha}$ for some $\alpha$. By renumbering the variables, we may assume $p \nmid j_{\text {od }}$.

We compute

$$
z=\partial_{J_{o}-\mathbf{e}_{d}} g=j_{o d} c_{J_{o}} \cdot x_{d}+\sum_{\alpha=1}^{d-1}\left(j_{o \alpha}+1\right) c_{J_{o}-\mathbf{e}_{d}+\mathbf{e}_{\alpha}} \cdot x_{\alpha} .
$$

Since $j_{o d} c_{J_{o}} \in k \backslash\{0\}$, we may take $\left(x_{1}, \ldots, x_{d-1}, z\right)$ as a new system of variables for the polynomial ring $G$. We set $G^{\prime}=k\left[x_{1}, \ldots, x_{d-1}\right]$ to be the polynomial ring with $(d-1)$-variables and $L^{\prime}=L \cap G^{\prime}$. Note that $L^{\prime}$ is $\mathfrak{D}$-saturated. Rewrite $g=\sum_{i=0}^{r^{\prime}} a_{i} z^{i}$ with $a_{i} \in G_{r^{\prime}-i}^{\prime}$ in terms of the new system of variables. 
Note that, for any $h \in G$ and $K \in \mathbb{Z}_{\geq 0}^{d}$, we have

$$
\partial_{X^{p^{s}}}\left(h^{p^{s}}\right)=\partial_{X^{K}}(h)^{p^{s}},
$$

which follows from the equations (cf. Remark 1.2.1.3 (2))

$$
\partial_{p^{s} K}\left(X^{p^{s} J}\right)=\left(\begin{array}{c}
p^{s} J \\
p^{s} K
\end{array}\right) X^{p^{s}(J-K)} \quad \text { and } \quad\left(\begin{array}{c}
p^{s} J \\
p^{s} K
\end{array}\right)=\left(\begin{array}{c}
J \\
K
\end{array}\right)=\left(\begin{array}{c}
J \\
K
\end{array}\right)^{p^{s}}
$$

Thus we have

$$
\left\{\begin{array}{l}
\partial_{X^{p^{s} K}}(f)=\partial_{X^{p^{s} K}}\left(g^{p^{s}}\right)=\partial_{X^{K}}(g)^{p^{s}}=z^{p^{s}}, \quad K=J_{o}-\mathbf{e}_{d}, \\
\partial_{z^{p^{s}}}(f)=\partial_{z^{p^{s}}}\left(g^{p^{s}}\right)=\partial_{z^{i}}(g)^{p^{s}}=a_{i}^{p^{s}}+\sum_{t=i+1}^{r^{\prime}}\left(\begin{array}{l}
t \\
i
\end{array}\right) a_{t}^{p^{s}} z^{(t-i) p^{s}} \quad\left(0 \leq i \leq r^{\prime}\right) .
\end{array}\right.
$$

Recall that $L$ is $\mathfrak{D}$-saturated. Thus the first formula implies $z^{p^{s}} \in L$, and the second formula implies $a_{i}^{p^{s}} \in L$ for $0 \leq i \leq r^{\prime}$ by descending induction on $i$.

On one hand, we have $z^{p^{s}} \in L^{\text {pure }}$ by definition. On the other hand, since $L^{\prime}=k\left[L^{\prime \text { pure }}\right]$ by inductional hypothesis on the number of variables, we have

$$
\left\{a_{i}^{p^{s}} ; 0 \leq i \leq r^{\prime}\right\} \subset L \cap G^{\prime}=L^{\prime}=k\left[L^{\prime \text { pure }}\right] \subset k\left[L^{\text {pure }}\right] .
$$

Therefore, we conclude

$$
f=g^{p^{s}}=\sum_{i=0}^{r^{\prime}} a_{i}^{p^{s}} z^{p^{s} i} \in k\left[L^{\text {pure }}\right] .
$$

This completes the inductional step and hence the proof for $L=k\left[L^{\text {pure }}\right]$.

In order to see the "Moreover" part of the statement, observe that there is a sequence of inclusions among the pure parts

$$
L_{p^{0}}^{\text {pure }}=L_{1}, \quad\left\{L_{p^{0}}^{\text {pure }}\right\}^{p} \subset L_{p^{1}}^{\text {pure }}, \quad\left\{L_{p^{1}}^{\text {pure }}\right\}^{p} \subset L_{p^{2}}^{\text {pure }}, \cdots .
$$

Let $e_{1}<\cdots<e_{i}<\cdots<e_{M}$ be the integers indicating the stages where the above inclusions are not equalities, i.e.,

$$
\begin{cases}\left\{L_{p^{e_{i}-1}}^{\text {pure }}\right\}^{p} \varsubsetneqq L_{p^{e_{i}}}^{\text {pure }} & (1 \leq i \leq M) \\ \left\{L_{p^{p^{e-1}}}^{\text {pure }}\right\}^{p}=L_{p^{e}}^{\text {pure }} & e \notin\left\{e_{i} ; 1 \leq i \leq M\right\} .\end{cases}
$$

Note that the set of such integers is a finite set, since the dimension of the pure part is uniformly bounded, i.e., $\operatorname{dim}_{k} L_{p^{e}}^{\text {pure }} \leq \operatorname{dim} G_{1}=d$ for any $e \in \mathbb{Z}_{\geq 0}$.

Now we have only to take $V_{i} \subset G_{1} \quad(i=1, \ldots, M)$ inductively so that

$$
F^{e_{i}}\left(V_{i}\right) \cup \bigcup_{j<i} F^{e_{i}}\left(V_{j}\right) \subset L_{p^{e_{i}}}^{\text {pure }}
$$


forms a basis of $L_{p^{e_{i}}}^{\text {pure }}$ for $1 \leq i \leq M$.

This completes the proof of Lemma 3.1.2.1.

3.1.3. Leading generator system. The statement of Lemma 3.1.2.1 leads us to the following notion of a leading generator system of a $\mathfrak{D}$-saturated idealistic filtration.

Definition 3.1.3.1. $\quad$ Let $\mathbb{I}$ be a $\mathfrak{D}$-saturated idealistic filtration. We call a subset $\mathbb{H}=\left\{\left(h_{i j}, p^{e_{i}}\right)\right\} \subset \mathbb{I}$ a leading generator system if it satisfies the following conditions:

(i) $h_{i j} \in \mathfrak{m}^{p^{e_{i}}}$ and $\overline{h_{i j}}=\left(h_{i j} \bmod \mathfrak{m}^{p^{e_{i}}+1}\right) \in L(\mathbb{I})_{p^{e_{i}}}^{\text {pure }}$ for any $i, j$,

(ii) $\left\{{\overline{h_{i j}}}^{p^{e-e_{i}}} ; e_{i} \leq e\right\}$ consists of $\#\left\{(i, j) ; e_{i} \leq e\right\}$-distinct elements, and forms a $k$-basis of $L(\mathbb{I})_{p^{e}}^{\text {pure }}$ for any $e \in \mathbb{Z}_{\geq 0}$.

Proposition 3.1.3.2. $\quad$ A leading generator system exists for a $\mathfrak{D}$ saturated idealistic filtration $\mathbb{I}$.

Proof. $\quad$ Since $\mathbb{I}$ is $\mathfrak{D}$-saturated, it follows that $L(\mathbb{I})$ is $\mathfrak{D}$-saturated and hence that we can apply Lemma 3.1.2.1 to $L=L(\mathbb{I}) \subset G$. Take a collection $\mathbb{H}=\left\{\left(h_{i j}, p^{e_{i}}\right)\right\}$ so that $\overline{h_{i j}}=v_{i j}^{p^{e_{i}}}$, where $\left\{e_{1}<\cdots<e_{M}\right\}$ and $V_{1} \sqcup \cdots \sqcup V_{M} \subset$ $G_{1}$ with $V_{i}=\left\{v_{i j}\right\}_{j}$ are taken as stated in Lemma 3.1.2.1, satisfying conditions (i) and (ii). Then $\mathbb{H}$ is a leading generator system for $\mathbb{I}$.

Remark 3.1.3.3.

(1) Condition (i) in Definition 3.1.3.1 can be rephrased in terms of the differential operators as follows:

(i) $D\left(h_{i j}\right) \in \mathfrak{m}$ for any $D \in \operatorname{Diff}_{R}^{\left(p^{e_{i}}\right)}$, where $\operatorname{Diff}_{R}^{\left(p^{e_{i}}\right)}$ is defined by the following formula

$$
\operatorname{Diff}_{R}^{\left(p^{e_{i}}\right)}:=\sum_{a+b=p^{e_{i}}, 0<a, b<p^{e_{i}}} \operatorname{Diff}_{R}^{a} \circ \operatorname{Diff}_{R}^{b} .
$$

(2) We often study a subset $\mathbb{H}=\left\{\left(h_{i j}, p^{e_{i}}\right)\right\} \subset \mathbb{I}$ which satisfies some slightly weaker conditions than those for a leading generator system. Namely, we require condition (i), and instead of full condition (ii) where $\left\{{\overline{h_{i j}}}^{p^{e-e_{i}}} ; e_{i} \leq e\right\}$ should form a basis of $L(\mathbb{I})_{p^{e}}^{\text {pure }}$, we only require $\left\{{\overline{h_{i j}}}^{p^{e-e_{i}}} ; e_{i} \leq e\right\}$ to be $k$ linearly independent. 
The class of the subsets described above, which is slightly bigger than the class of the leading generator systems, is often better suited for the purpose of setting up some inductional proofs. We refer the reader to Chapter 4 for the examples of such proofs.

\section{§3.2. Invariants $\sigma$ and $\tilde{\mu}$}

In this section, we present the definitions of the two of the most basic invariants (at the closed point $P \in W$ ) that we use in our algorithm, $\sigma$ and $\widetilde{\mu}$, in relation to the notion of a leading generator system.

We warn the reader, however, that in the actual process of our algorithm, the definitions of $\sigma$ and $\widetilde{\mu}$ will be slightly modified. For example, in order to determine the invariant $\tilde{\mu}$ in our setting, we also have to take the boundary divisor of reference into consideration, just as we do to determine the weak order in the classical setting.

The purpose of this presentation is to bring a flavor of how these invariants may function in our algorithm, while the details, including their fundamental properties, will be discussed in Parts II, III, and IV.

\subsubsection{Invariant $\sigma$.}

Definition 3.2.1.1. $\quad$ Let $\mathbb{I}$ be a $\mathfrak{D}$-saturated idealistic filtration. Then the invariant $\sigma$ is defined to be the following infinite sequence indexed by $e \in$ $\mathbb{Z}_{\geq 0}$

$$
\sigma=\left(d-l_{p^{0}}^{\text {pure }}, d-l_{p^{1}}^{\text {pure }}, \ldots, d-l_{p^{e}}^{\text {pure }}, \ldots\right)
$$

where

$$
d=\operatorname{dim} W, \quad l_{p^{e}}^{\text {pure }}=\operatorname{dim}_{k} L(\mathbb{I})_{p^{e}}^{\text {pure }} .
$$

More precisely, $\sigma$ should be considered as a function $\sigma: \mathbb{Z}_{\geq 0} \rightarrow \mathbb{Z}_{\geq 0}$ defined by

$$
\sigma(e)=d-l_{p^{e}}^{\text {pure }} .
$$

Remark 3.2.1.2.

(1) The reason why we take the (infinite) sequence of $d-l_{p^{e}}^{\text {pure }}$ instead of the (infinite) sequence of $l_{p^{e}}^{\text {pure }}$ is two-fold:

(i) When we consider the invariant $l_{p^{e}}^{\text {pure }}$, fixing $e$ but varying $P \in W$, we see (cf. Part II) that it is lower semi-continuous. Taking its negative, we have our invariant upper semi-continuous as desired. 
(In other words, the bigger $l_{p^{e}}^{\text {pure }}$ is, the better the singularities are. Therefore, as the measure of how bad the singularities are, it is natural to take our invariant to be its negative.)

(ii) We reduce the problem of resolution of singularities of a variety $X$ to that of embedded resolution. Therefore, it would be desirable or even necessary to come up with an algorithm which would induce the "same" process of resolution of singularities, no matter what ambient variety $W$ we choose for an embedding $X \hookrightarrow W$ (locally).

While $l_{p^{e}}^{\text {pure }}$ is dependent of the choice of $W, \operatorname{dim} W-l_{p^{e}}^{\text {pure }}$ is not. Therefore, the latter is more appropriate as an invariant toward constructing such an algorithm.

(2) As observed before, the dimension of the pure part is non-decreasing (with respect to the power $e \in \mathbb{Z}_{\geq 0}$ of $p^{e}$ ), and is uniformly bounded from above by $d=\operatorname{dim} W$, i.e.,

$$
0 \leq l_{p^{0}}^{\text {pure }} \leq l_{p^{1}}^{\text {pure }} \leq \cdots l_{p^{e-1}}^{\text {pure }} \leq l_{p^{e}}^{\text {pure }} \leq \cdots \leq d=\operatorname{dim} W
$$

and hence stabilizes after some point, i.e., there exists $e_{M} \in \mathbb{Z}_{\geq 0}$ such that for $e>e_{M}$ the above inequalities become equalities

$$
l_{p^{e-1}}^{\text {pure }}=l_{p^{e}}^{\text {pure }} .
$$

Therefore, though $\sigma$ is an infinite sequence by definition, essentially we are only looking at some finite part of it.

(3) In characteristic zero, the invariant $\sigma$ consists of only one term $(d-\operatorname{dim}$ $\left.L(\mathbb{I})_{1}\right)$, where $\operatorname{dim} L(\mathbb{I})_{1}$ can be regarded as indicating "how many linearly independent hypersurfaces of maximal contact we can take" for $\mathbb{I}$.

(4) We relieve ourselves of the task of giving the precise definition of the " $\tau$-invariant" in the theory of Hironaka's additive group scheme (cf. [Hir70] [Oda73] [Oda83] [Oda87]). However, roughly speaking, Hironaka's $\tau$-invariant in our context corresponds to the total number of the elements in a leading generator system $\# \mathbb{H}=\#\left\{\left(h_{i j}, p^{e_{i}}\right)\right\}$. That is to say, in terms of Remark (2), it is equal to $l_{p^{e} M}^{\text {pure }}$. Our invariant $\sigma$, which incorporates the information on the levels of the elements in a leading generator system, although closely related and inspired by the ideas of Hironaka, is different from Hironaka's $\tau$-invariant. 


\subsubsection{Invariant $\widetilde{\mu}$.}

Definition 3.2.2.1. $\quad$ Let $\mathbb{I}$ be a $\mathfrak{D}$-saturated idealistic filtration. Take a leading generator system $\mathbb{H}=\left\{\left(h_{i j}, p^{e_{i}}\right)\right\}$ of $\mathbb{I}$. Set $\mathcal{H}=\left\{h_{i j}\right\}$ and $(\mathcal{H}) \subset R$ to be the ideal generated by $\mathcal{H}$.

For $f \in R$, we define its multiplicity (or order) modulo $(\mathcal{H})$ to be

$$
\operatorname{ord}_{\mathcal{H}}(f)=\sup \left\{n \in \mathbb{Z}_{\geq 0} ; f \in \mathfrak{m}^{n}+(\mathcal{H})\right\}
$$

We also define

$$
\mu_{\mathcal{H}}(\mathbb{I}):=\inf \left\{\mu_{\mathcal{H}}(f, a):=\frac{\operatorname{ord}_{\mathcal{H}}(f)}{a} ;(f, a) \in \mathbb{I}, a \in \mathbb{R}_{>0}\right\} .
$$

(Note that we set $\operatorname{ord}_{\mathcal{H}}(0)=\infty$ by definition.) Finally we define the invariant $\widetilde{\mu}$ by

$$
\widetilde{\mu}=\mu_{\mathcal{H}}(\mathbb{I}) .
$$

Remark 3.2.2.2.

(1) We will see in Part II that $\mu_{\mathcal{H}}(\mathbb{I})$ is independent of the choice of a leading generator system, and hence that the invariant $\widetilde{\mu}$ is actually an intrinsic one associated to the idealistic filtration $\mathbb{I}$.

(2) In characteristic zero, where $\mathcal{H}$ forms (a part of) a regular system of parameters, the upper semi-continuity of the invariant $\widetilde{\mu}$ (along the locus where the invariant $\sigma$ is constant) follows immediately from the upper semi-continuity of the usual multiplicity defined on the nonsingular subvariety defined by the ideal $(\mathcal{H})$. In positive characteristic, however, it is highly non-trivial, and its verification is one of the main subjects of Part II.

(3) In our algorithm, the invariant $\widetilde{\mu}$ is actually computed as $\mu_{\mathcal{H}, E}(\mathbb{I})$, using not only the information about a leading generator system but also the one about the boundary divisor $E$ in reference. For all the details, we refer the reader to Parts II, III, and IV.

(4) In Part II, we study the power series expansion of $f \in R$ with respect to (the elements in $\mathcal{H}$ associated to) a leading generator system. There the invariant $\operatorname{ord}_{\mathcal{H}}(f)$ can be computed as the multiplicity of the "constant" term. Again we refer the reader to Part II for its detail.

(5) In characteristic zero, the invariant $\widetilde{\mu}$ corresponds to the multiplicity of what is called the coefficient ideal (restricted to a hypersurface of maximal contact) in the classical setting. 
(6) In characteristic zero (or more generally in the case where all the elements of a leading generator system are concentrated at level 1, i.e., in the case where $e_{i}=0$ for any $i$ ), the order modulo $(\mathcal{H})$ is additive in the sense that for $f, g \in R$ we have

$$
\operatorname{ord}_{\mathcal{H}}(f g)=\operatorname{ord}_{\mathcal{H}}(f)+\operatorname{ord}_{\mathcal{H}}(g) .
$$

In positive characteristic $\operatorname{char}(k)=p>0$, however, we have only the inequality

$$
\operatorname{ord}_{\mathcal{H}}(f g) \geq \operatorname{ord}_{\mathcal{H}}(f)+\operatorname{ord}_{\mathcal{H}}(g)
$$

in general, and the strict inequality does actually happen in the case $e_{i}>0$ for some $i$. It is possible that, for $(f, a / b) \in \mathbb{I}$ with $a, b \in \mathbb{Z}_{>0}$ and hence $\left(f^{b}, a\right) \in \mathbb{I}$, we have

$$
\mu_{\mathcal{H}}\left(f^{b}, a\right)=\frac{\operatorname{ord}_{\mathcal{H}}\left(f^{b}\right)}{a}>\frac{b \cdot \operatorname{ord}_{\mathcal{H}}(f)}{a}=\frac{\operatorname{ord}_{\mathcal{H}}(f)}{a / b}=\mu_{\mathcal{H}}(f, a / b) .
$$

It is also possible that

$$
\inf \left\{\mu_{\mathcal{H}}(f, a) ;(f, a) \in \mathbb{I}, a \in \mathbb{Z}_{>0}\right\}>\inf \left\{\mu_{\mathcal{H}}(f, a) ;(f, a) \in \mathbb{I}, a \in \mathbb{R}_{>0}\right\} .
$$

Therefore, it makes a real difference in computing the invariant $\widetilde{\mu}$ whether we only consider the integral levels or we consider the rational (and real) levels (cf. the last emphasis in 0.2.3.1. and Remark 2.2.3.3). We refer the reader to Part II for more details.

\section{Chapter 4. Nonsingularity Principle}

The purpose of this chapter is to establish the nonsingularity principle, which guarantees the nonsingularity of the center of each blowup in our algorithm (cf. 0.2.3.2.4.). Recall that in the classical setting the nonsingularity of the center is guaranteed by the nonsingularity of a hypersurface of maximal contact (cf. 0.2.1.3.). Since we use a leading generator system as a collective substitute for a hypersurface of maximal contact, and since a leading generator system may contain some elements defining singular hypersurfaces, the classical nonsingularity principle is no longer available in our algorithm. Instead, we look at the final form of the idealistic filtration, obtained after the modifications associated with the construction of the strand of invariants (cf. 0.2.3.2.2.). It has a special property of having the invariant $\tilde{\mu}=\infty$. What we prove is that an idealistic filtration with invariant $\tilde{\mu}=\infty$, assuming it is both $\mathfrak{R}$-saturated and $\mathfrak{D}$-saturated (i.e., $\mathfrak{B}$-saturated), has all the elements in a leading generator 
system concentrated at level 1 , and hence that its support, defined by these elements, is nonsingular. Therefore, we conclude that the center, which is the maximum locus of the strand of the invariants and which coincides with the support of the idealistic filtration described as above, is also nonsingular. We emphasize that the use of the $\mathfrak{R}$-saturation, as seen in the nonsingular principle, is indispensable in our algorithm. This feature seems to distinguish our algorithm from the other proposed approaches (cf. 0.5.2), where the $\mathfrak{R}$-saturation (integral closure) may appear in their conceptual foundation but is never used in the actual algorithm.

The following is the brief description of this chapter.

In Section 4.1, we prepare some technical lemmas that we use in the proof of the nonsingularity principle. They describe the behavior of a leading generator system, which we expect to be parallel to the behavior of a collection of hypersurfaces of maximal contact forming (a part of) a regular system of parameters in characteristic zero. We will use these lemmas again later in our series of papers.

Section 4.2 , where we present the statement and proof of the nonsingularity principle, is literally the culminating point of Part I.

In this Section, $R$ represents the localization at a maximal ideal, or its completion, of the coordinate ring of an affine open subset of a variety $W$ smooth over an algebraically closed field $k$ of $\operatorname{char}(k)=p>0$, or characteristic zero where we formally set $p=\infty$ in the arguments below. We denote by $\mathfrak{m}$ the maximal ideal of $R$, which corresponds to a closed point $P \in W$.

\section{§4.1. Preparation toward the nonsingularity principle}

4.1.1. Setting for the supporting lemmas. We fix the following setting for the three supporting lemmas we present in 4.1.2:

Let $\mathcal{H}=\left\{h_{1}, \ldots, h_{N}\right\} \subset R$ be a subset of $R$ consisting of $N$ elements, and let $0 \leq e_{1} \leq \cdots \leq e_{N}$ be nonnegative integers associated to these elements, satisfying the following conditions:

(i) $h_{l} \in \mathfrak{m}^{p^{e_{l}}}$ and $\overline{h_{l}}=\left(h_{l} \bmod \mathfrak{m}^{p^{e_{l}}+1}\right) \in F^{e_{l}}\left(G_{1}\right)$ for $l=1, \ldots, N$. (See Definition 3.1.1.1.)

(ii) $\left\{{\overline{h_{l}}}^{p^{e_{s}-e_{l}}} ; e_{l} \leq e_{s}\right\}$ consists of $\#\left\{l ; e_{l} \leq e_{s}\right\}$-distinct and $k$-linearly independent elements in the $k$-vector space $F^{e_{s}}\left(G_{1}\right)$ for $s=1, \ldots, N$. 
We set

$$
\left\{\begin{array}{l}
e:=e_{1}=\min \left\{e_{l} ; l=1, \ldots, N\right\} \\
L:=\max \left\{l ; l=1, \ldots, N, e_{l}=e\right\}=\#\left\{l ; l=1, \ldots, N, e_{l}=e\right\} \\
e^{\prime}:=e_{L+1} \quad\left(\text { if } L=N, \text { then we set } e^{\prime}=\infty\right)
\end{array}\right.
$$

Let $\left(x_{1}, \ldots, x_{d}\right)$ be a regular system of parameters for $R$ such that

$$
M_{d, L}=\left[\partial_{x_{i}^{p^{e}}}\left(h_{l}\right)\right]_{l=1, \ldots, L}^{i=1, \ldots, d} \in M(d \times L, R)
$$

has the invertible $L \times L$ first minor, i.e.,

$$
M=\left[\partial_{x_{i}^{p^{e}}}\left(h_{l}\right)\right]_{l=1, \ldots, L}^{i=1, \ldots, L} \in \mathrm{GL}(L, R)
$$

Let $C=\left[c_{i j}\right]_{j=1, \ldots, L}^{i=1, \ldots, L} \in \operatorname{GL}(L, R)$ be the inverse matrix of $M$ so that $C M=I_{L}$.

We introduce the following multi-index notations:

$$
T:=\left(t_{1}, \ldots, t_{L}\right) \in \mathbb{Z}_{\geq 0}^{L}, \quad c^{T}:=\prod_{j=1}^{L}\left(c_{L, j}\right)^{t_{j}} .
$$

Remark 4.1.1.1.

(1) Condition (i) in Setting 4.1.1 can be rephrased in terms of the differential operators as follows (cf. Remark 3.1.3.3 (1)):

(i) $D\left(h_{l}\right) \in \mathfrak{m} \quad \forall D \in \operatorname{Diff}_{R}^{\left(p^{e_{l}}\right)}$.

(2) We are not assuming in our situation that the subset $\mathcal{H}$ is associated to a leading generator system of an idealistic filtration. See Remark 3.1.3.3 (2).

(3) Conditions (i) and (ii) imply that, for any regular system of parameters $\left(y_{1}, \ldots, y_{d}\right)$, the matrix $M_{y}=\left[\partial_{y_{i}^{p}}\left(h_{l}\right)\right]_{l=1, \ldots, L}^{i=1, \ldots, d}$ is of size $d \times L$ and has the full rank, i.e., rank $M_{y}=L$. Therefore, by a linear change of variables, we may always come up with a regular system of parameters $\left(x_{1}, \ldots, x_{d}\right)$ satisfying the condition in our situation.

4.1.2. Statements and proofs of the supporting lemmas. Given a regular system of parameters $\left(x_{1}, \ldots, x_{d}\right)$, we have the corresponding partial differential operators $\partial_{x_{i}^{u}} \quad\left(u \in \mathbb{Z}_{>0}\right)$ for $1 \leq i \leq d$. Given a set of elements $\left(h_{1}, \ldots, h_{N}\right)$ as described in the setting (e.g. the set associated to a leading 
generator system), we would like to have their corresponding partial differential operators. The next lemma constructs a differential operator $D_{u}$, which behaves like " $\partial_{h_{L}^{u}}$ " in spirit when we look at the initial terms of our concern.

Lemma 4.1.2.1 (Supporting Lemma 1). Let u, $r$ be integers such that

$$
0 \leq u<p^{e^{\prime}-e} \quad \text { and } \quad 0 \leq r .
$$

Define

$$
D_{u}:=\sum_{|T|=u} c^{T} \partial_{p^{e} T} \in \operatorname{Diff}_{R}^{u p^{e}} \text { and } D_{-1}=0,
$$

where we use the abbreviated notation $\partial_{J}=\partial_{X^{J}}$.

Then for any $\beta \in \mathfrak{m}^{r}$ and $1 \leq l \leq N$, we have

$$
D_{u}\left(\beta h_{l}\right) \equiv\left(D_{u} \beta\right) h_{l}+\delta_{L, l} D_{u-1} \beta \quad \bmod \mathfrak{m}^{r+p^{e_{l}}-u p^{e}+1}
$$

Proof. By the generalized product rule, we have

$$
D_{u}\left(\beta h_{l}\right)=\sum_{|T|=u} c^{T} \partial_{p^{e} T}\left(\beta h_{l}\right)=\sum_{|T|=u} c^{T} \sum_{J \leq p^{e} T}\left(\partial_{p^{e} T-J} \beta\right)\left(\partial_{J} h_{l}\right) .
$$

We remark here that

$$
\left\{p^{e_{l}} \not J\right\} \text { or }\left\{p^{e_{l}}<|J|\right\} \Longrightarrow \operatorname{ord}_{P}\left(\partial_{J} h_{l}\right)>p^{e_{l}}-|J| .
$$

Thus, in the process of continuing the above computation modm $\mathfrak{m}^{r+p^{e} l}-u p^{e}+1$, the term $\partial_{J} h_{l}$ is relevant only when $J=p^{e_{l}} \mathbf{e}_{j}(1 \leq j \leq L)$ or when $J=\mathbf{O}$. Therefore, we have

$$
D_{u}\left(\beta h_{l}\right) \equiv\left(\sum_{|T|=u} c^{T} \partial_{p^{e} T} \beta\right) h_{l}+\sum_{j=1}^{L} \sum_{|T|=u} c^{T}\left(\partial_{p^{e} T-p^{e} l \mathbf{e}_{j}} \beta\right)\left(\partial_{p^{e} l} \mathbf{e}_{j} h_{l}\right)
$$

where the first and the second term in the right hand side correspond to the case $J=\mathbf{O}$ and $J=p^{e_{l}} \mathbf{e}_{j}$ for $1 \leq j \leq L$ respectively.

We remark here that in the generalized product rule we only consider the case where $\mathbf{O} \leq p^{e} T-p^{e_{l}} \mathbf{e}_{j}$. Looking at the $j$-th components, we conclude

$$
0 \leq p^{e^{e}} t_{j}-p^{e_{l}} \leq p^{e}|T|-p^{e_{l}}<p^{e} \cdot p^{e^{\prime}-e}-p^{e_{l}}=p^{e^{\prime}}-p^{e_{l}}
$$

This implies that we only consider the case where

$$
e_{l}=e \quad \text { and } \quad t_{j} \geq 1 .
$$


Therefore, setting $T^{\prime}=T-\mathbf{e}_{j}$, we have

$$
\begin{aligned}
D_{u}\left(\beta h_{l}\right) & \equiv\left(D_{u} \beta\right) h_{l}+\delta_{e, e_{l}} \sum_{j=1}^{L} \sum_{\left|T^{\prime}\right|=u-1} c^{T^{\prime}+\mathbf{e}_{j}}\left(\partial_{p^{e} T^{\prime}} \beta\right)\left(\partial_{p^{e} \mathbf{e}_{j}} h_{l}\right) \\
& =\left(D_{u} \beta\right) h_{l}+\delta_{e, e_{l}}\left(\sum_{j=1}^{L} c_{L, j} \partial_{p^{e} \mathbf{e}_{j}} h_{l}\right)\left(\sum_{\left|T^{\prime}\right|=u-1} c^{T^{\prime}} \partial_{p^{e} T^{\prime}} \beta\right) \\
& =\left(D_{u} \beta\right) h_{l}+\delta_{e, e_{l}}(C M)_{L, l} D_{u-1} \beta \\
& =\left(D_{u} \beta\right) h_{l}+\delta_{L, l} D_{u-1} \beta .
\end{aligned}
$$

Therefore, we conclude

$$
D_{u}\left(\beta h_{l}\right) \equiv\left(D_{u} \beta\right) h_{l}+\delta_{L, l} D_{u-1} \beta \quad \bmod \mathfrak{m}^{r+p^{e_{l}}-u p^{e}+1} .
$$

This completes the proof of Lemma 4.1.2.1.

The next lemma computes the coefficient of $h_{L}$, using the differential operator constructed in the previous lemma, in terms of the coefficients of the other elements $h_{l}(l \neq L)$ and terms of higher multiplicity.

Lemma 4.1.2.2 (Supporting Lemma 2). Let $v, s$ be integers such that

$$
1 \leq v<p^{e^{\prime}-e} \quad \text { and } \quad 0 \leq s .
$$

Define

$$
F_{v}=\sum_{u=1}^{v}(-1)^{u} h_{L}^{u-1} D_{u} .
$$

Suppose that the elements $\alpha, \beta_{1}, \ldots, \beta_{N} \in R$ satisfy the following conditions:

$$
\left\{\begin{array}{l}
\alpha+\sum_{l=1}^{N} \beta_{l} h_{l} \in \mathfrak{m}^{s+1} \\
\operatorname{ord}_{P}\left(\beta_{l}\right) \geq s-p^{e_{l}} \quad(1 \leq l \leq N) .
\end{array}\right.
$$

Then we have

$$
\beta_{L} \equiv F_{v} \alpha+(-1)^{v} h_{L}^{v} D_{v} \beta_{L}+\sum_{\substack{1 \leq l \leq N \\ l \neq L}}\left(F_{v} \beta_{l}\right) h_{l} \quad \bmod \mathfrak{m}^{s-p^{e}+1}
$$

(We use the convention that $\mathfrak{m}^{n}=R$ when $n \leq 0$.) 
Proof. From Supporting Lemma 1 it follows that for $1 \leq l \leq N$

$$
D_{u}\left(\beta_{l} h_{l}\right) \equiv\left(D_{u} \beta_{l}\right) h_{l}+\delta_{L, l} D_{u-1} \beta_{l} \quad \bmod \mathfrak{m}^{s-u p^{e}+1} .
$$

Multiplying by $(-1)^{u} h_{L}^{u-1}$ and adding them up with $u$ ranging from 1 to $v$, we obtain

$$
\begin{aligned}
F_{v}\left(\beta_{l} h_{l}\right) & \equiv\left(F_{v} \beta_{l}\right) h_{l}+\delta_{L, l} \sum_{u=1}^{v}(-1)^{u} h_{L}^{u-1} D_{u-1} \beta_{l} \quad \bmod \mathfrak{m}^{s-p^{e}+1} \\
& =\left(F_{v} \beta_{l}\right) h_{l}-\delta_{L, l} \sum_{u=0}^{v-1}(-1)^{u} h_{L}^{u} D_{u} \beta_{l}
\end{aligned}
$$

Since $\alpha+\sum_{l=1}^{N} \beta_{l} h_{l} \in \mathfrak{m}^{s+1}$, we have $F_{v}\left(\alpha+\sum_{l=1}^{N} \beta_{l} h_{l}\right) \in \mathfrak{m}^{s+1-p^{e}}$.

Therefore, we obtain

$$
\begin{aligned}
F_{v} \alpha & \equiv-F_{v}\left(\sum_{l=1}^{N} \beta_{l} h_{l}\right) \bmod \mathfrak{m}^{s+1-p^{e}} \\
& \equiv-\sum_{l=1}^{N}\left\{\left(F_{v} \beta_{l}\right) h_{l}-\delta_{L, l} \sum_{u=0}^{v-1}(-1)^{u} h_{L}^{u} D_{u} \beta_{l}\right\} \bmod \mathfrak{m}^{s+1-p^{e}} \\
& =-\sum_{l=1}^{N}\left(F_{v} \beta_{l}\right) h_{l}+\sum_{u=0}^{v-1}(-1)^{u} h_{L}^{u} D_{u} \beta_{L} .
\end{aligned}
$$

Therefore, we conclude

$$
\begin{aligned}
& F_{v} \alpha+\sum_{\substack{1 \leq l \leq N \\
l \neq L}}\left(F_{v} \beta_{l}\right) h_{l} \equiv-\left(F_{v} \beta_{L}\right) h_{L}+\sum_{u=0}^{v-1}(-1)^{u} h_{L}^{u} D_{u} \beta_{L} \quad \bmod \mathfrak{m}^{s+1-p^{e}} \\
& =-\sum_{u=1}^{v}(-1)^{u} h_{L}^{u} D_{u} \beta_{L}+\sum_{u=0}^{v-1}(-1)^{u} h_{L}^{u} D_{u} \beta_{L}=D_{0} \beta_{L}-(-1)^{v} h_{L}^{v} D_{v} \beta_{L} .
\end{aligned}
$$

Since $D_{0}=\mathrm{id}_{R}$, we conclude

$$
\beta_{L} \equiv F_{v} \alpha+(-1)^{v} h_{L}^{v} D_{v} \beta_{L}+\sum_{\substack{1 \leq l \leq N, l \neq L}}\left(F_{v} \beta_{l}\right) h_{l} \quad \bmod \mathfrak{m}^{s-p^{e}+1}
$$

This completes the proof of Lemma 4.1.2.2.

The next lemma shows that, given a linear combination of $\left(h_{1}, \ldots, h_{L}\right)$, we can retake the coefficients so that they have the expected multiplicities. 
This paves the way to the coefficient lemma in the next subsection, where we extract more information on the coefficients when $\left(h_{l}, p^{e_{l}}\right) \quad(l=1, \ldots, N)$ are in a ( $\mathfrak{D}$-saturated) idealistic filtration.

Lemma 4.1.2.3 (Supporting Lemma 3). We have

$$
\left(\sum_{l=1}^{N} R h_{l}\right) \cap \mathfrak{m}^{r}=\sum_{l=1}^{N} \mathfrak{m}^{r-p^{e_{l}}} h_{l} \text { for any } r \in \mathbb{Z}_{\geq 0} .
$$

(We use the convention that $\mathfrak{m}^{n}=R$ when $n \leq 0$.)

Proof. We have only to show the inclusion

$$
(\diamond) \quad\left(\sum_{l=1}^{N} R h_{l}\right) \cap \mathfrak{m}^{r} \subset \sum_{l=1}^{N} \mathfrak{m}^{r-p^{e_{l}}} h_{l},
$$

while the opposite one is clear.

We prove the inclusion by induction on the triplet $(\chi, L, r)$ where

$$
\chi=\left\{e_{l} ; l=1, \ldots, N\right\},
$$

and where the set of the triplets is endowed with the lexicographical order.

Case 1. $(\chi, L)=(1,1)$, i.e., $N=1$.

In this case, take $\beta h_{1} \in\left(R h_{1}\right) \cap \mathfrak{m}^{r}$ with $\beta \in\left(\mathfrak{m}^{r}: h_{1}\right)$. Then since $h_{1} \notin \mathfrak{m}^{p^{e}+1}$, we have $\beta \in \mathfrak{m}^{r-p^{e}}$. Thus we have

$$
\left(R h_{1}\right) \cap \mathfrak{m}^{r}=\left(\mathfrak{m}^{r}: h_{1}\right) h_{1} \subset \mathfrak{m}^{r-p^{e}} h_{1}
$$

which shows the inclusion $(\diamond)$. (Note that the inclusion $(\diamond)$ holds even when $r<p^{e}$.)

$$
\text { Case 2. }(\chi, L)>(1,1), r \leq p^{e_{N}} .
$$

In this case, set $M=\min \left\{l ; e_{l}=e_{N}\right\}$. Since $r \leq p^{e_{N}}$, we observe

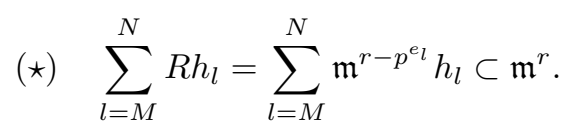

Assume $\chi=1$. Then we have $M=1$, and $(\star)$ implies the inclusion $(\diamond)$ immediately. 
Assume $\chi>1$. Then we we have

$$
\begin{aligned}
\left(\sum_{l=1}^{N} R h_{l}\right) \cap \mathfrak{m}^{r} & =\left(\sum_{l=1}^{M-1} R h_{l}+\sum_{l=M}^{N} R h_{l}\right) \cap \mathfrak{m}^{r} \\
& =\left(\sum_{l=1}^{M-1} R h_{l}\right) \cap \mathfrak{m}^{r}+\sum_{l=M}^{N} \mathfrak{m}^{r-p^{e_{l}}} h_{l} \quad(\text { by }(\star)) \\
& \subset \sum_{l=1}^{M-1} \mathfrak{m}^{r-p^{e_{l}}} h_{l}+\sum_{l=M}^{N} \mathfrak{m}^{r-p^{e_{l}}} h_{l}=\sum_{l=1}^{N} \mathfrak{m}^{r-p^{e_{l}}} h_{l},
\end{aligned}
$$

which implies the inclusion $(\diamond)$. Note that the inclusion on the third line is obtained by induction on $\chi$, since

$$
\#\left\{e_{l} ; 1 \leq l \leq M-1\right\}=\chi-1 .
$$

Case 3. $(\chi, L)>(1,1), r>p^{e_{N}}$.

Note that this case happens only when we are in positive characteristic $0<$ $p=\operatorname{char}(k)<\infty$. In this case, we take an element

$$
g=\sum_{l=1}^{N} \beta_{l} h_{l} \in\left(\sum_{l=1}^{N} R h_{l}\right) \cap \mathfrak{m}^{r} \subset\left(\sum_{l=1}^{N} R h_{l}\right) \cap \mathfrak{m}^{r-1} .
$$

By induction on $r$, we may assume

$$
\beta_{l} \in \mathfrak{m}^{r-1-p^{e_{l}}} \quad(1 \leq l \leq N) .
$$

By applying Supporting Lemma 2 with

$$
0<p^{e^{\prime}-e}-1=v, \quad 0 \leq r-1=s, \quad \alpha=0,
$$

as we check the conditions

$$
\alpha+\sum_{l=1}^{N} \beta_{l} h_{l} \in \mathfrak{m}^{s+1}, \quad \operatorname{ord}_{P}\left(\beta_{l}\right) \geq s-p^{e_{l}} \quad(1 \leq l \leq N),
$$

we conclude

$$
\beta_{L} \equiv F_{v} \alpha+(-1)^{v} h_{L}^{v} D_{v} \beta_{L}+\sum_{\substack{1 \leq l \leq N, l \neq L}}\left(F_{v} \beta_{l}\right) h_{l} \quad \bmod \mathfrak{m}^{s-p^{e}+1} .
$$

Since $F_{v} \alpha=0$, we conclude

$$
\beta_{L} \in R h_{L}^{p^{e^{\prime}-e}-1}+\sum_{\substack{1 \leq l \leq N, l \neq L}} R h_{l}+\mathfrak{m}^{r-p^{e}} .
$$


Therefore, we have

$$
\begin{aligned}
g & =\sum_{l=1}^{N} \beta_{l} h_{l}=\sum_{\substack{1 \leq l \leq N, l \neq L}} \beta_{l} h_{l}+\beta_{L} h_{L} \\
& \in\left(\sum_{\substack{1 \leq l \leq N, l \neq L}} R h_{l}+R h_{L}^{p^{e^{\prime}-e}}+\mathfrak{m}^{r-p^{e}} h_{L}\right) \cap \mathfrak{m}^{r} \\
& =\left(\sum_{\substack{1 \leq l \leq N, l \neq L}} R h_{l}+R h_{L}^{p^{e^{\prime}-e}}\right) \cap \mathfrak{m}^{r}+\mathfrak{m}^{r-p^{e}} h_{L} .
\end{aligned}
$$

Now instead of looking at the original

$$
\mathcal{H}=\left\{h_{1}, \ldots, h_{L-1}, h_{L}, h_{L+1}, \ldots, h_{N}\right\} \quad \text { with }(\chi, L),
$$

we look at

$$
\mathcal{H}^{\prime}=\left\{h_{1}, \ldots, h_{L-1}, h_{L}^{\prime}=h_{L}^{p^{e^{\prime}-e}}, h_{L+1}, \ldots, h_{N}\right\} \quad \text { with }\left(\chi^{\prime}, L^{\prime}\right) .
$$

If $L=1$, then we have $\chi^{\prime}=\chi-1$. If $L>1$, then we have $\left(\chi^{\prime}, L^{\prime}\right)=(\chi, L-1)$. Hence we always have $\left(\chi^{\prime}, L^{\prime}\right)<(\chi, L)$. Therefore, by induction, we conclude

$$
\left(\sum_{\substack{1 \leq l \leq N, l \neq L}} R h_{l}+R h_{L}^{p^{e^{\prime}-e}}\right) \cap \mathfrak{m}^{r} \subset \sum_{\substack{1 \leq l \leq N, l \neq L}} \mathfrak{m}^{r-p^{e_{l}}} h_{l}+\mathfrak{m}^{r-p^{e^{\prime}}} h_{L}^{p^{e^{\prime}-e}} .
$$

Plugging in this inclusion for the third line of the analysis for $g$, we conclude

$$
\begin{aligned}
g & \in\left(\sum_{\substack{1 \leq l \leq N, l \neq L}} R h_{l}+R h_{L}^{p^{e^{\prime}-e}}\right) \cap \mathfrak{m}^{r}+\mathfrak{m}^{r-p^{e}} h_{L} . \\
& =\sum_{\substack{1 \leq l \leq N, l \neq L}} \mathfrak{m}^{r-p^{e_{l}}} h_{l}+\mathfrak{m}^{r-p^{e^{\prime}}} h_{L}^{p^{e^{\prime}-e}}+\mathfrak{m}^{r-p^{e}} h_{L} \\
& =\sum_{\substack{1 \leq l \leq N, l \neq L}} \mathfrak{m}^{r-p^{e_{l}}} h_{l}+\mathfrak{m}^{r-p^{e}} h_{L}=\sum_{l=1}^{N} \mathfrak{m}^{r-p^{e_{l}}} h_{l} .
\end{aligned}
$$


Since $g \in\left(\sum_{l=1}^{N} R h_{l}\right) \cap \mathfrak{m}^{r}$ is arbitrary, we have the inclusion

$$
(\diamond) \quad\left(\sum_{l=1}^{N} R h_{l}\right) \cap \mathfrak{m}^{r} \subset \sum_{l=1}^{N} \mathfrak{m}^{r-p^{e}{ }_{l}} h_{l} .
$$

This completes the proof of Lemma 4.1.2.3.

4.1.3. Setting for the coefficient lemma. We describe the setting for the coefficient lemma:

Let $\mathbb{I}$ be a $\mathfrak{D}$-saturated idealistic filtration over $R$.

Let $\mathcal{H}=\left\{h_{1}, \ldots, h_{N}\right\} \subset R$ be a subset of $R$, and let $0 \leq e_{1} \leq \cdots \leq e_{N}$ be nonnegative integers associated to these elements, satisfying conditions (i) and (ii) as described in Setting 4.1.1, and satisfying one more condition (iii) $\left(h_{l}, p^{e_{l}}\right) \in \mathbb{I}$ for $l=1, \ldots, N$.

We denote by $(\mathcal{H}) \subset R$ the ideal generated by the set $\mathcal{H}$.

For $f \in R$, set

$$
\operatorname{ord}_{\mathcal{H}}(f)=\sup \left\{n \in \mathbb{Z}_{\geq 0} ; f \in \mathfrak{m}^{n}+(\mathcal{H})\right\}
$$

and

$$
\mu_{\mathcal{H}}(\mathbb{I}):=\inf \left\{\mu_{\mathcal{H}}(f, a):=\frac{\operatorname{ord}_{\mathcal{H}}(f)}{a} ;(f, a) \in \mathbb{I}, a \in \mathbb{R}_{>0}\right\} .
$$

Note that we set $\operatorname{ord}_{\mathcal{H}}(0)=\infty$ by definition.

We also bring the attention of the reader to the following notation:

For $B=\left(b_{1}, \ldots, b_{N}\right) \in \mathbb{Z}_{\geq 0}^{N}$, we set $[B]=\left(b_{1} p^{e_{1}}, \ldots, b_{N} p^{e_{N}}\right)$ and hence

$$
|[B]|=\sum_{l=1}^{N} b_{l} p^{e_{l}} .
$$

\subsubsection{Statement and proof of the coefficient lemma.}

Lemma 4.1.4.1 (Coefficient Lemma). Let $\nu \in \mathbb{R}_{\geq 0}$ be a nonnegative number such that $\nu<\mu_{\mathcal{H}}(\mathbb{I})$. Set

$$
\mathbb{I}_{t}^{\prime}=\mathbb{I}_{t} \cap \mathfrak{m}^{\lceil\nu t\rceil},
$$

where we use the convention that $\mathfrak{m}^{n}=R$ for $n \leq 0$. Then for any $a \in \mathbb{R}$, we have

$$
\mathbb{I}_{a}=\sum_{B} \mathbb{I}_{a-|[B]|}^{\prime} H^{B}
$$


Proof. We set

$$
\mathfrak{q}_{a}=\sum_{B} \mathbb{I}_{a-|[B]|}^{\prime} H^{B} \subset \mathbb{I}_{a} .
$$

Our goal is to show $\mathbb{I}_{a}=\mathfrak{q}_{a}$.

When $a \leq 0$, since $R=\mathbb{I}_{a}^{\prime} \subset \mathfrak{q}_{a}$, we have $\mathbb{I}_{a}=R=\mathfrak{q}_{a}$, the desired equality.

Therefore, in the following, we assume $a>0$.

Step 1. Proof for the inclusion $(\star)_{c, r}$ defined below.

For $c \in \mathbb{Z}_{>0}$ and $r \in \mathbb{Z}_{\geq 0}$, we set

$$
J_{c, r}=\mathfrak{m}^{r+1}+\mathfrak{q}_{a}+\sum_{|[B]| \geq c} \mathfrak{m}^{r-|[B]|} H^{B} .
$$

We prove the inclusion

$$
(\star)_{c, r} \quad \mathbb{I}_{a} \cap \mathfrak{m}^{r} \subset J_{c, r} \quad\left(1 \leq c \leq\lceil a\rceil, r \in \mathbb{Z}_{\geq 0}\right)
$$

by induction on $c$.

Case 1. $c=1$.

In this case, if $\lceil\nu a\rceil \leq r$, then the inclusion $(\star)_{1, r}$ holds since

$$
\mathbb{I}_{a} \cap \mathfrak{m}^{r} \subset \mathbb{I}_{a} \cap \mathfrak{m}^{\lceil\nu a\rceil}=\mathbb{I}_{a}^{\prime} \subset \mathfrak{q}_{a} \subset J_{1, r} .
$$

If $\lceil\nu a\rceil \geq r+1$, then we have

$$
\begin{aligned}
\mathbb{I}_{a} \cap \mathfrak{m}^{r} & \left.\subset\left(\mathfrak{m}^{\lceil\nu a\rceil}+(\mathcal{H})\right) \cap \mathfrak{m}^{r} \quad \text { (by definition of } \mu_{\mathcal{H}}(\mathbb{I}) \text { and } \nu\right) \\
& \subset \mathfrak{m}^{r+1}+\left(\sum_{l=1}^{N} R_{l} h_{l}\right) \cap \mathfrak{m}^{r} \quad\left(\text { since } \mathfrak{m}^{\lceil\nu a\rceil} \subset \mathfrak{m}^{r+1} \subset \mathfrak{m}^{r}\right) \\
& =\mathfrak{m}^{r+1}+\sum_{l=1}^{N} \mathfrak{m}^{r-p^{e_{l}}} h_{l} \quad(\text { by Supporting Lemma 3) } \\
& =\mathfrak{m}^{r+1}+\sum_{|B|=1} \mathfrak{m}^{r-|[B]|} H^{B} \subset \mathfrak{m}^{r+1}+\sum_{|[B]| \geq 1} \mathfrak{m}^{r-|[B]|} H^{B} \subset J_{1, r},
\end{aligned}
$$

and hence the inclusion $(\star)_{1, r}$.

Case 2. $\quad c \geq 2$ assuming the inclusion $(\star)_{c-1, r}$.

Using the inclusion $(\star)_{c-1, r}$, we have

$$
\begin{aligned}
\mathbb{I}_{a} \cap \mathfrak{m}^{r} & \subset\left(\mathfrak{m}^{r+1}+\mathfrak{q}_{a}+\sum_{|[B]| \geq c-1} \mathfrak{m}^{r-|[B]|} H^{B}\right) \cap \mathbb{I}_{a} \\
& =\mathfrak{q}_{a}+\left(\mathfrak{m}^{r+1}+\sum_{|[B]| \geq c-1} \mathfrak{m}^{r-|[B]|} H^{B}\right) \cap \mathbb{I}_{a}
\end{aligned}
$$


Since $\mathfrak{q}_{a} \subset J_{c, r}$, in order to show the inclusion $(\star)_{c, r}$, we have only to prove

$$
\left(\mathfrak{m}^{r+1}+\sum_{|[B]| \geq c-1} \mathfrak{m}^{r-|[B]|} H^{B}\right) \cap \mathbb{I}_{a} \subset J_{c, r} .
$$

Let $f$ be an element in the left-hand side of the desired inclusion above, so that there exists a finite set

$$
\left\{\alpha_{B} \in \mathfrak{m}^{r-|[B]|} ;|[B]| \geq c-1\right\} \subset R
$$

such that

$$
f-\sum_{|[B]| \geq c-1} \alpha_{B} H^{B} \in \mathfrak{m}^{r+1} .
$$

Fix a multi-index $B_{o}$ with $\left|\left[B_{o}\right]\right|=c-1$.

Choose a regular system of parameters $\left(x_{1}, \ldots, x_{d}\right)$ such that

$$
h_{l}-x_{l}^{p^{e_{l}}} \in \mathfrak{m}^{p^{e_{l}}+1} \quad(1 \leq l \leq N) .
$$

The partial derivatives in the following computation are taken with respect to this regular system of parameters $X=\left(x_{1}, \ldots, x_{d}\right)$. We use the abbreviation $\partial_{J}=\partial_{X^{J}}$. The symbol "三" denotes an equality modulo $\mathfrak{m}^{r-(c-1)+1}=\mathfrak{m}^{r-c+2}$. We compute

$$
\begin{aligned}
\partial_{\left[B_{o}\right]} f & \equiv \sum_{|[B]| \geq c-1} \partial_{\left[B_{o}\right]}\left(\alpha_{B} H^{B}\right) \\
& =\sum_{|[B]| \geq c-1} \sum_{J \leq\left[B_{o}\right]}\left(\partial_{\left[B_{o}\right]-J} \alpha_{B}\right)\left(\partial_{J} H^{B}\right) \quad \text { (by the generalized product rule) } \\
& \equiv \sum_{|[B]| \geq c-1} \sum_{J \leq\left[B_{o}\right]}\left(\partial_{\left[B_{o}\right]-J} \alpha_{B}\right)\left(\partial_{J} X^{[B]}\right) \\
& =\sum_{|[B]| \geq c-1} \sum_{J \leq\left[B_{o}\right]}\left(\partial_{\left[B_{o}\right]-J} \alpha_{B}\right)\left(\begin{array}{c}
{[B]} \\
J
\end{array}\right) X^{[B]-J} .
\end{aligned}
$$

In the last formula, the binomial coefficient $\left(\begin{array}{c}{[B]} \\ J\end{array}\right)$ is zero unless $J=[K]$ for some $K \leq B_{o}$. Therefore, we have

$$
\begin{aligned}
\partial_{\left[B_{o}\right]} f & \equiv \sum_{|[B]| \geq c-1} \sum_{K \leq B_{o}}\left(\partial_{\left[B_{o}-K\right]} \alpha_{B}\right)\left(\begin{array}{l}
{[B]} \\
{[K]}
\end{array}\right) X^{[B-K]} \\
& =\sum_{|[B]| \geq c-1} \sum_{K \leq B_{o}}\left(\partial_{\left[B_{o}-K\right]} \alpha_{B}\right)\left(\begin{array}{l}
B \\
K
\end{array}\right) X^{[B-K]} \\
& \equiv \sum_{|[B]| \geq c-1} \sum_{K \leq B_{o}}\left(\partial_{\left[B_{o}-K\right]} \alpha_{B}\right)\left(\begin{array}{l}
B \\
K
\end{array}\right) H^{B-K} .
\end{aligned}
$$


In the last formula, the binomial coefficient $\left(\begin{array}{c}B \\ K\end{array}\right)=0$ unless $K \leq B$.

If $K<B$, we have $|[B-K]| \geq 1$ and

$$
\partial_{\left[B_{o}-K\right]} \alpha_{B} \in \mathfrak{m}^{r-|[B]|-\left|\left[B_{o}-K\right]\right|}=\mathfrak{m}^{r-(c-1)-|[B-K]|} .
$$

If $K=B$, we have $B=B_{o}$, since $B=K \leq B_{o}$ and $\left|\left[B_{o}\right]\right|=c-1 \leq|[B]|$.

Therefore, we have

$$
\begin{aligned}
(*) \partial_{\left[B_{o}\right]} f-\alpha_{B_{o}} & \in \sum_{K<B} \mathfrak{m}^{r-c+1-|[B-K]|} H^{B-K}+\mathfrak{m}^{r-c+2} \\
& =\sum_{|[B]| \geq 1} \mathfrak{m}^{r-c+1-|[B]|} H^{B}+\mathfrak{m}^{r-c+2} .
\end{aligned}
$$

On the other hand, since $f \in \mathbb{I}_{a} \cap \mathfrak{m}^{r}$ and since the idealistic filtration $\mathbb{I}$ is $\mathfrak{D}$-saturated, we have

$$
\partial_{\left[B_{o}\right]} f \in \mathbb{I}_{a-(c-1)} \cap \mathfrak{m}^{r-(c-1)}=\mathbb{I}_{a-c+1} \cap \mathfrak{m}^{r-c+1} .
$$

Using the inclusion $(\star)_{1, r-c+1}$, we obtain

$$
(* *) \quad \partial_{\left[B_{o}\right]} f \in \mathbb{I}_{a-c+1} \cap \mathfrak{m}^{r-c+1} \subset \mathfrak{m}^{r-c+2}+\mathfrak{q}_{a-c+1}+\sum_{|[B]| \geq 1} \mathfrak{m}^{r-c+1-|[B]|} H^{B} .
$$

From $(*)$ and $(* *)$ it follows that

$$
\begin{aligned}
\alpha_{B_{o}} H^{B_{o}} & \in \mathfrak{m}^{r-c+2} H^{B_{o}}+\mathfrak{q}_{a-c+1} H^{B_{o}}+\sum_{|[B]| \geq 1} \mathfrak{m}^{r-c+1-|[B]|} H^{B+B_{o}} \\
& \subset \mathfrak{m}^{r+1}+\mathfrak{q}_{a}+\sum_{\left|\left[B+B_{o}\right]\right| \geq c} \mathfrak{m}^{r-c+1-|[B]|} H^{B+B_{o}} \subset J_{c, r} .
\end{aligned}
$$

Since $B_{o}$ is arbitrary with $\left|\left[B_{o}\right]\right|=c-1$, we conclude that $\alpha_{B} H^{B} \in J_{c, r}$ for all $B$ with $|[B]|=c-1$. Therefore, we have

$$
f \in \sum_{|[B]| \geq c-1} \alpha_{B} H^{B}+\mathfrak{m}^{r+1}=\sum_{|[B]|=c-1} \alpha_{B} H^{B}+\left(\mathfrak{m}^{r+1}+\sum_{|[B]| \geq c} \alpha_{B} H^{B}\right) \subset J_{c, r},
$$

which implies the desired inclusion $(\star)_{c, r}$.

This completes the proof of the inclusion $(\star)_{c, r}$.

Step 2. Finishing argument.

We finish the proof of Coefficient Lemma using the result of Step 1.

Applying the inclusion $(\star)_{\lceil a\rceil, r}$ for $r \in \mathbb{Z}_{\geq 0}$, we have

$$
\mathbb{I}_{a} \cap \mathfrak{m}^{r} \subset \mathfrak{m}^{r+1}+\mathfrak{q}_{a}+\sum_{|[B]| \geq\lceil a\rceil} \mathfrak{m}^{r-|[B]|} H^{B}=\mathfrak{m}^{r+1}+\mathfrak{q}_{a},
$$


since $\mathbb{I}_{a-|[B]|}^{\prime}=R$ for $B$ with $|[B]| \geq\lceil a\rceil$.

Therefore, we have

$$
\mathbb{I}_{a} \cap \mathfrak{m}^{r} \subset \mathbb{I}_{a} \cap\left(\mathfrak{m}^{r+1}+\mathfrak{q}_{a}\right)=\mathbb{I}_{a} \cap \mathfrak{m}^{r+1}+\mathfrak{q}_{a},
$$

which implies

$$
\mathbb{I}_{a} \cap \mathfrak{m}^{r}+\mathfrak{q}_{a}=\mathbb{I}_{a} \cap \mathfrak{m}^{r+1}+\mathfrak{q}_{a},
$$

for any $r \in \mathbb{Z}_{\geq 0}$. In particular, we have

$$
\mathbb{I}_{a}=\mathbb{I}_{a} \cap \mathfrak{m}^{0}+\mathfrak{q}_{a}=\mathbb{I}_{a} \cap \mathfrak{m}^{\lceil\nu a\rceil}+\mathfrak{q}_{a}=\mathbb{I}_{a}^{\prime}+\mathfrak{q}_{a}=\mathfrak{q}_{a} .
$$

This completes the proof of Lemma 4.1.4.1.

Remark 4.1.4.2.

(1) The purpose of having a nonnegative number $\nu \in \mathbb{R}_{\geq 0}$ with $\nu<\mu_{\mathcal{H}}(\mathbb{I})$ involved in our statement of Lemma 4.1.4.1 is to make it valid even when $\mu_{\mathcal{H}}(\mathbb{I})=\infty$, the case to which we often apply Coefficient Lemma. When $\mu_{\mathcal{H}}(\mathbb{I})<\infty$, we may of course apply Coefficient Lemma, setting $\nu=\mu_{\mathcal{H}}(\mathbb{I})$.

(2) We can restrict the range of $B$ in the expression $\mathbb{I}_{a}=\sum_{B} \mathbb{I}_{a-|[B]|}^{\prime} H^{B}$ to a specific finite range, e.g., $B$ with $|[B]|<a+p^{e_{N}}$. In fact, if $|[B]| \geq a+p^{e_{N}}$, there exists $B^{\prime}<B$ such that $a \leq\left|\left[B^{\prime}\right]\right|<a+p^{e_{N}}$. Then we have $\mathbb{I}_{a-|[B]|}^{\prime} H^{B} \subset$ $R H^{B^{\prime}}=\mathbb{I}_{a-\left|\left[B^{\prime}\right]\right|}^{\prime} H^{B^{\prime}}$. Therefore, if $B$ is out of this range, the term $\mathbb{I}_{a-|[B]|}^{\prime} H^{B}$ is redundant, i.e.,

$$
\sum_{B} \mathbb{I}_{a-|[B]|}^{\prime} H^{B}=\sum_{|[B]|<a+p^{e} N} \mathbb{I}_{a-|[B]|}^{\prime} H^{B} .
$$

(3) In Part II, given an element $(f, a) \in \mathbb{I}$ of a $\mathfrak{D}$-saturated idealistic filtration, we analyze "the power series expansion of $f$ with respect to a set $\mathcal{H}$ satisfying conditions (i), (ii), (iii) (e.g. a leading generator system of $\mathbb{I}$ )". This provides a different approach to Coefficient Lemma and an alternative proof.

\section{§4.2. Nonsingularity principle}

\subsubsection{Statement of the nonsingularity principle.}

Theorem 4.2.1.1. $\quad$ Let $\mathbb{I}$ be an idealistic filtration which is $\mathfrak{B}$-saturated. Let $\mathcal{H}=\left\{h_{1}, \ldots, h_{N}\right\} \subset R$ be a subset of $R$, and let $0 \leq e_{1} \leq \cdots \leq e_{N}$ be nonnegative integers associated to these elements, satisfying conditions (i), (ii), (iii) as described in Setting 4.1.3. Suppose $\mu_{\mathcal{H}}(\mathbb{I})=\infty$. Then 
(1) $\mathbb{H}=\left\{\left(h_{l}, p^{e_{l}}\right) ; l=1, \ldots, N\right\}$ generates the idealistic filtration $\mathbb{I}$, i.e.,

$$
\mathbb{I}=G(\mathbb{H}) .
$$

(2) The elements in $\mathbb{H}$ are all concentrated at level $p^{0}=1$, i.e.,

$$
\mathbb{H} \subset R \times\{1\} .
$$

(Note that in characteristic zero, where we take $p=\infty$ according to our convention, we set $p^{0}=\infty^{0}=1$. cf. 0.2.3.2.1.).

Consequently, we conclude that the support of the idealistic filtration is defined by $\mathcal{H}$, i.e., $\operatorname{Supp}(\mathbb{I})=V(\mathcal{H})$, and hence that it is nonsingular.

Remark 4.2.1.2.

(1) In Theorem 4.2.1.1, we see from assertion (1) that

$$
\left\{\overline{h_{l}}=\left(h_{l} \bmod \mathfrak{m}^{p^{e_{l}}}\right) ; l=1, \ldots, N\right\}
$$

generates $L(\mathbb{I})$ (cf. Definition 3.1.1.1), and hence conclude that $\mathbb{H}$ is a leading generator system, even though we do not a priori assume so.

(2) In Part II, we will look at the invariant $\widetilde{\mu}$, which is a priori defined to be $\widetilde{\mu}=\mu_{\mathcal{H}}(\mathbb{I})$ with respect to the set $\mathcal{H}$ associated to a leading generator system. We will see, however, that $\mu_{\mathcal{H}}(\mathbb{I})$ is independent of the choice of a leading generator system, and hence that $\widetilde{\mu}$ is actually an invariant intrinsic to the idealistic filtration $\mathbb{I}$. Therefore, the nonsingularity principle above can be regarded as the description of an idealistic filtration with $\widetilde{\mu}=\infty$, with the conclusions holding for any leading generator system $\mathbb{H}$.

(3) Recall that, as we construct the strand of invariants in our algorithm, we enlarge the idealistic filtration and construct its modifications (cf. 0.2.3.2.2. and 0.2 .3 .2 .4 .). At the end of the construction of the strand of invariants, we reach the last modification, which is an idealistic filtration (which is both $\mathfrak{R}$-saturated and $\mathfrak{D}$-saturated) whose leading generator system satisfies the conditions described in the above. The maximum locus of the strand of invariants, which we take as the center of blowup, coincides with the support of this last modification (in a neighborhood of each point of the maximum locus), and hence is nonsingular according to Theorem 4.2.1.1. This is why it is called the nonsingularity principle of the center.

(4) In order to show $\mathbb{I}=G(\mathbb{H})$, we only need $\mathbb{I}$ to be $\mathfrak{D}$-saturated, while in order to show $\mathbb{H} \subset R \times\{1\}$, we need $\mathbb{I}$ to be $\mathfrak{B}$-saturated. 


\subsubsection{Proof of the nonsingularity principle.}

Proof for assertion (1).

We show that $\mathbb{H}$ generates the idealistic filtration $\mathbb{I}$, i.e., $\mathbb{I}=G(\mathbb{H})$.

Since $\mu_{\mathcal{H}}(\mathbb{I})=\infty$, we can apply Coefficient Lemma with an arbitrary nonnegative number $\mathbb{Z}_{\geq 0} \ni \nu<\mu_{\mathcal{H}}(\mathbb{I})=\infty$ and obtain

$$
\begin{aligned}
\mathbb{I}_{a} & =\sum_{B} \mathbb{I}_{a-|[B]|}^{\prime} H^{B}=\sum_{|[B]| \geq a} \mathbb{I}_{a-|[B]|}^{\prime} H^{B}+\sum_{|[B]|<a} \mathbb{I}_{a-|[B]|}^{\prime} H^{B} \\
& \subset \sum_{|[B]| \geq a} R H^{B}+\mathbb{I}_{a-(\lceil a\rceil-1)}^{\prime} \subset \sum_{|[B]| \geq a} R H^{B}+\mathfrak{m}^{\lceil\nu(a-\lceil a\rceil+1)\rceil} .
\end{aligned}
$$

Since $a-\lceil a\rceil+1>0$, this implies by Krull's intersection theorem that

$$
\mathbb{I}_{a} \subset \bigcap_{r=0}^{\infty}\left(\sum_{|[B]| \geq a} R H^{B}+\mathfrak{m}^{r}\right)=\sum_{|[B]| \geq a} R H^{B} .
$$

This shows that $\mathbb{H}$ generates $\mathbb{I}$, i.e., $\mathbb{I}=G(\mathbb{H})$.

Proof for assertion (2).

We show that the elements in $\mathbb{H}$ are concentrated at level $p^{0}=1$, i.e., $\mathbb{H} \subset R \times\{1\}$. Set

$$
\mathbb{H}_{0}=\left\{\left(h_{l}, p^{e_{l}}\right) \in \mathbb{H} ; e_{l}=0\right\}=\mathbb{H} \cap(R \times\{1\}) .
$$

We will derive a contradiction assuming $\mathbb{H}_{0} \neq \mathbb{H}$. Set $e=\min \left\{e_{l} ; e_{l}>0\right\}$.

Step 1. We show that $\mathbb{I}_{a}=(\mathcal{H})$ for $0<a \leq 1$ and that $(\mathcal{H})=\sqrt{(\mathcal{H})}$. In fact, since $\mathbb{I}=G(\mathbb{H})$ and since $\mathbb{H} \subset R \times \mathbb{R}_{\geq 1}$, Lemma 2.2.1.2 (1) implies that

$$
\mathbb{I}_{a}=\sum_{l=1}^{N} R h_{l}=(\mathcal{H}) \quad \text { for } \quad 0<a \leq 1 .
$$

Suppose $g \in \sqrt{(\mathcal{H})}$, i.e., $g^{n} \in(\mathcal{H})=\mathbb{I}_{1}$ for some $n \in \mathbb{Z}_{>0}$. Since $\mathbb{I}$ is $\mathfrak{R}$ saturated, this implies $g \in \mathbb{I}_{1 / n}=(\mathcal{H})$. Therefore, we have $(\mathcal{H})=\sqrt{(\mathcal{H})}$.

Step 2. We show that $(\mathcal{H})=\left((\mathcal{H}) \cap R^{p^{e}}\right)+\left(\mathcal{H}_{0}\right)$.

Set

$$
\mathcal{D}=\left\{d \in \operatorname{Diff}_{R}^{p^{e}-1} ; d\left(\left(\mathcal{H}_{0}\right)\right) \subset\left(\mathcal{H}_{0}\right)\right\}
$$

Observe

$$
\text { (*) } \mathcal{D}((\mathcal{H})) \subset(\mathcal{H}) .
$$

In fact, for $d \in \mathcal{D}$, since $\mathcal{H} \backslash \mathcal{H}_{0} \subset \bigcup_{e_{l}>0} \mathbb{I}_{p^{e_{l}}}=\mathbb{I}_{p^{e}}$ and since $\mathbb{I}$ is $\mathfrak{D}$-saturated, we have

$$
d\left(\left(\mathcal{H} \backslash \mathcal{H}_{0}\right)\right) \subset d\left(\mathbb{I}_{p^{e}}\right) \subset \mathbb{I}_{p^{e}-\left(p^{e}-1\right)}=\mathbb{I}_{1}=(\mathcal{H}) .
$$


Therefore, we conclude

$$
d((\mathcal{H}))=d\left(\left(\mathcal{H}_{0}\right)\right)+d\left(\left(\mathcal{H} \backslash \mathcal{H}_{0}\right)\right) \subset\left(\mathcal{H}_{0}\right)+(\mathcal{H})=(\mathcal{H}) .
$$

Now $(*)$ implies

$$
(* *) \quad \operatorname{Diff}_{\bar{R}}^{p^{e}-1}(\overline{(\mathcal{H})}) \subset \overline{(\mathcal{H})} \text { and hence } \operatorname{Diff} \overline{p^{e}}-1(\overline{(\mathcal{H})})=\overline{(\mathcal{H})}
$$

where

$$
\bar{R}=R /\left(\mathcal{H}_{0}\right) \quad \text { and } \quad \overline{(\mathcal{H})}=(\mathcal{H}) /\left(\mathcal{H}_{0}\right)
$$

Then, by Proposition 1.3.1.2, (**) implies

$$
\overline{(\mathcal{H})}=\left(\overline{(\mathcal{H})} \cap \bar{R}^{p^{e}}\right) .
$$

Therefore, we have

$$
(\mathcal{H})=\left((\mathcal{H}) \cap R^{p^{e}}\right)+\left(\mathcal{H}_{0}\right)
$$

Step 3. Finishing argument.

By Step 2, we conclude

$$
\begin{aligned}
(\mathcal{H}) & =\left(\mathcal{H}_{0}\right)+\left((\mathcal{H}) \cap R^{p^{e}}\right)=\left(\mathcal{H}_{0}\right)+\left(\left\{g^{p^{e}} \in(\mathcal{H}) ; g \in R\right\}\right) \\
& =\left(\mathcal{H}_{0}\right)+\left(\left\{g^{p^{e}} ; g \in(\mathcal{H})\right\}\right) \quad(\text { by }(\mathcal{H})=\sqrt{(\mathcal{H})}) \\
& =\left(\mathcal{H}_{0}\right)+\left(\left\{g^{p^{e}} ; g \in\left(\mathcal{H} \backslash \mathcal{H}_{0}\right)\right\}\right) \subset\left(\mathcal{H}_{0}\right)+\mathfrak{m}^{p^{e}+1},
\end{aligned}
$$

i.e., $(\mathcal{H}) \subset\left(\mathcal{H}_{0}\right)+\mathfrak{m}^{p^{e}+1}$.

Choose a regular system of parameters $\left(x_{1}, \ldots, x_{d}\right)$ so that

$$
\left\{\begin{array}{l}
x_{l}=h_{l} \text { for } 1 \leq l \leq L \text { where } L=\#\left\{l ; e_{l}=0\right\} \\
x_{l}^{p^{e_{l}}} \equiv h_{l} \bmod \mathfrak{m}^{p^{e}+1} \quad \text { for } L+1 \leq l \leq N .
\end{array}\right.
$$

Then the above inclusion would imply

$$
\left\{(\mathcal{H})+\mathfrak{m}^{p^{e}+1}\right\} / \mathfrak{m}^{p^{e}+1} \subset\left\{\left(\mathcal{H}_{0}\right)+\mathfrak{m}^{p^{e}+1}\right\} / \mathfrak{m}^{p^{e}+1} \subset R / \mathfrak{m}^{p^{e}+1}
$$

and we identify $R / \mathfrak{m}^{p^{e}+1} \cong k\left[x_{1}, \ldots, x_{d}\right] /\left(x_{1}, \ldots, x_{d}\right)^{p^{e}+1}$.

On the other hand, however, we have the following element in the first quotient

(the leading term of $\left.h_{L+1}\right)=x_{L+1}^{p^{e_{L+1}}}=x_{L+1}^{p^{e}} \in\left\{(\mathcal{H})+\mathfrak{m}^{p^{e}+1}\right\} / \mathfrak{m}^{p^{e}+1}$, 
which obviously is not in the middle quotient

$$
\left(x_{1}, \ldots, x_{L}\right)=\left\{\left(\mathcal{H}_{0}\right)+\mathfrak{m}^{p^{e}+1}\right\} / \mathfrak{m}^{p^{e}+1},
$$

a contradiction!

This contradiction is derived from the assumption that $\mathbb{H}_{0} \neq \mathbb{H}$.

Therefore, we conclude $\mathbb{H}_{0}=\mathbb{H}$, i.e.,

$$
\mathbb{H} \subset R \times\{1\} .
$$

This completes the proof of Theorem 4.2.1.1, the nonsingularity principle.

\section{References}

[Abh66] S. S. Abhyankar, Resolution of singularities of embedded algebraic surfaces, Academic Press, New York, 1966.

[Abh77] L Lectures on expansion techniques in algebraic geometry, Tata Inst. Fund. Res., Bombay, 1977.

[Abh83] - Desingularization of plane curves, in Singularities, Part 1 (Arcata, Calif., 1981), 1-45, Proc. Sympos. Pure Math., 40, Amer. Math. Soc., Providence, RI.

[AdJ97] D. Abramovich and A. J. de Jong, Smoothness, semistability, and toroidal geometry, J. Algebraic Geom. 6 (1997), no. 4, 789-801.

[AHV75] J. M. Aroca, H. Hironaka and J. L. Vicente, The theory of the maximal contact, Inst. "Jorge Juan" Mat., Consejo Superior de Investigaciones Cientificas, Madrid, 1975 .

[AHV77] _ Desingularization theorems, Consejo Sup. Inv. Cient., Madrid, 1977.

[Ben70] B. M. Bennett, On the characteristic functions of a local ring, Ann. of Math. (2) 91 (1970), 25-87.

[BEV05] A. M. Bravo, S. Encinas and O. Villamayor U., A simplified proof of desingularization and applications, Rev. Mat. Iberoamericana 21 (2005), no. 2, 349-458.

[Bie04] E. Bierstone, Resolution of singularities. A preprint for series of lectures at the "Workshop on resolution of singularities, factorization of birational mappings, and toroidal geometry" at Banff International Research Station, Banff, during 11-16 December, 2004.

[Bie05] - Functoriality in resolution of singularities, Slides for a colloquium at Harvard University on November 10, 2005.

[BM89] E. Bierstone and P. D. Milman, Uniformization of analytic spaces, J. Amer. Math. Soc. 2 (1989), no. 4, 801-836.

[BM90] L Local resolution of singularities, in Real analytic and algebraic geometry (Trento, 1988), 42-64, Lecture Notes in Math., 1420, Springer, Berlin.

[BM91] _ A simple constructive proof of canonical resolution of singularities, in Effective methods in algebraic geometry (Castiglioncello, 1990), 11-30, Progr. Math., 94, Birkhäuser, Boston, Boston, MA.

[BM97] Canonical desingularization in characteristic zero by blowing up the maximum strata of a local invariant, Invent. Math. 128 (1997), no. 2, 207-302.

[BM99] , Resolution of singularities, in Several complex variables (Berkeley, CA, 1995-1996), 43-78, Cambridge Univ. Press, Cambridge.

[BM03] , Desingularization algorithms. I. Role of exceptional divisors, Mosc. Math. J. 3 (2003), no. 3, 751-805, 1197. 
[BM07] E. Bierstone and P. D. Milman, Functoriality in resolution of singularities. preprint, http://arXiv.org/abs/math.AG/0702375, 2007.

[Bou64] N. Bourbaki, Éléments de mathématique. Fasc. XXX. Algèbre commutative. Chapitre 5: Entiers. Chapitre 6: Valuations, Hermann, Paris, 1964.

[BP96] F. A. Bogomolov and T. G. Pantev, Weak Hironaka theorem, Math. Res. Lett. 3 (1996), no. 3, 299-307.

[BV03] A. M. Bravo and O. Villamayor U., A strengthening of resolution of singularities in characteristic zero, Proc. London Math. Soc. (3) 86 (2003), no. 2, 327-357.

[Cos87] V. Cossart, Forme normale d'une fonction sur un $k$-schéma de dimension 3 et de caractéristique positive, in Géométrie algébrique et applications, I (La Rábida, 1984), 1-21, Hermann, Paris.

[Cos00] Uniformisation et désingularisation des surfaces d'après Zariski, in Resolution of singularities (Obergurgl, 1997), 239-258, Progr. Math., 181, Birkhäuser, Basel.

[Cos04] $\longrightarrow$, Towards local uniformization along a valuation in Artin-Schreier extensions (dimension 3). A talk at the "Workshop on resolution of singularities, factorization of birational mappings, and toroidal geometry" at Banff International Research Station, Banff, during 11-16 December, 2004.

[Cut04] S. D. Cutkosky, Resolution of singularities, Amer. Math. Soc., Providence, RI, 2004.

[Cut06] _ Resolution of singularities for 3-folds in positive characteristic. preprint, http://arxiv.org/abs/math. AG/0606530, 2006.

[dJ96] A. J. de Jong, Smoothness, semi-stability and alterations, Inst. Hautes Études Sci. Publ. Math. No. 83 (1996), 51-93.

[EH02] S. Encinas and H. Hauser, Strong resolution of singularities in characteristic zero, Comment. Math. Helv. 77 (2002), no. 4, 821-845.

[ENV03] S. Encinas, A. Nobile and O. Villamayor U., On algorithmic equi-resolution and stratification of Hilbert schemes, Proc. London Math. Soc. (3) 86 (2003), no. 3, 607-648.

[EV98] S. Encinas and O. Villamayor, Good points and constructive resolution of singularities, Acta Math. 181 (1998), no. 1, 109-158.

[EV00] - A course on constructive desingularization and equivariance, in Resolution of singularities (Obergurgl, 1997), 147-227, Progr. Math., 181, Birkhäuser, Basel.

[EV03] A new proof of desingularization over fields of characteristic zero, Rev. Mat. Iberoamericana 19 (2003), no. 2, 339-353.

[EV07] _ Rees algebras and resolution of singularities. preprint, http://arXiv . org/abs/math. AG/0702836, 2007.

[Gir74] J. Giraud, Sur la théorie du contact maximal, Math. Z. 137 (1974), 285-310.

[Gir75] Contact maximal en caractéristique positive, Ann. Sci. École Norm. Sup. (4) 8 (1975), no. 2, 201-234.

[Gir83] , Forme normale d'une fonction sur une surface de caractéristique positive, Bull. Soc. Math. France 111 (1983), no. 2, 109-124.

[Gro67] A. Grothendieck, Éléments de géométrie algébrique. IV. Étude locale des schémas et des morphismes de schémas IV, Inst. Hautes Études Sci. Publ. Math. No. 32 (1967), $361 \mathrm{pp}$.

[Hau98] H. Hauser, Seventeen obstacles for resolution of singularities, in Singularities (Oberwolfach, 1996), 289-313, Progr. Math., 162, Birkhäuser, Basel.

[Hau03] $\_$, The Hironaka theorem on resolution of singularities (or: A proof we always wanted to understand), Bull. Amer. Math. Soc. (N.S.) 40 (2003), no. 3, 323-403 (electronic).

[Hir63] H. Hironaka, On resolution of singularities (characteristic zero), in Proc. Internat. Congr. Mathematicians (Stockholm, 1962), 507-521, Inst. Mittag-Leffler, Djursholm. 
[Hir64] H. Hironaka, Resolution of singularities of an algebraic variety over a field of characteristic zero. I, II, Ann. of Math. (2) 79 (1964), 109-203; ibid. (2) 79 (1964), 205-326.

[Hir70] Additive groups associated with points of a projective space, Ann. of Math. (2) 92 (1970), 327-334.

[Hir72a] _ Gardening of infinitely near singularities, in Algebraic geometry, Oslo 1970 (Proc. Fifth Nordic Summer School in Math.), 315-332, Wolters-Noordhoff, Groningen.

[Hir72b] _ Schemes, etc, in Algebraic geometry, Oslo 1970 (Proc. Fifth Nordic Summer School in Math.), 291-313, Wolters-Noordhoff, Groningen.

[Hir77] _ Idealistic exponents of singularity, in Algebraic geometry (J. J. Sylvester Sympos., Johns Hopkins Univ., Baltimore, Md., 1976), 52-125, Johns Hopkins Univ. Press, Baltimore, Md.

[Hir03] _ Theory of infinitely near singular points, J. Korean Math. Soc. 40 (2003), no. 5, 901-920.

[Hir05] _ Three key theorems on infinitely near singularities, in Singularités Franco-Japonaises, 87-126, Soc. Math. France, Paris.

[Hir06] _ A program for resolution of singularities, in all characteristics $p>0$ and in all dimensions. preprint for series of lectures in "Summer School on Resolution of Singularities" at International Center for Theoretical Physics, Trieste, during 12-30 June, 2006.

[HLOQ00] H. Hauser, J. Lipman, F. Oort and A. Quirós, editors. Resolution of singularities, volume 181 of Progress in Mathematics. Birkhäuser Verlag, Basel, 2000. A research textbook in tribute to Oscar Zariski, Papers from the Working Week held in Obergurgl, September 7-14, 1997.

[Kol05] J. Kollár, Resolution of singularities - Seattle lecture. notes for series of lectures given in the AMS Summer Institute, http://arxiv.org/abs/math.AG/0508332, 2005

[Kuh97] F.-V. Kuhlmann, On local uniformization in arbitrary characteristic. The Fields Institute Preprint Series, 1997.

[Kuh00] _ Valuation theoretic and model theoretic aspects of local uniformization, in Resolution of singularities (Obergurgl, 1997), 381-456, Progr. Math., 181, Birkhäuser, Basel.

[Lip69] J. Lipman, Rational singularities, with applications to algebraic surfaces and unique factorization, Inst. Hautes Études Sci. Publ. Math. No. 36 (1969), 195279.

[Lip75] Introduction to resolution of singularities, in Algebraic geometry (Proc. Sympos. Pure Math., Vol. 29, Humboldt State Univ., Arcata, Calif., 1974), 187230, Amer. Math. Soc., Providence, R.I.

[Lip78] , Desingularization of two-dimensional schemes. Ann. Math. (2) 107 (1978), no. 1, 151-207.

[Lip83] J. Lipman, Quasi-ordinary singularities of surfaces in $\mathbf{C}^{3}$, in Singularities, Part 2 (Arcata, Calif., 1981), 161-172, Proc. Sympos. Pure Math., 40, Amer. Math. Soc., Providence, RI.

[LT74] M. Lejeune-Jalabert and B. Teissier, Clôture intégrale des idéaux et équisingularité. Séminaire sur les singularité à l'Ecole Polytechnique, pages 1-66, 1974-1975.

[Mat86] H. Matsumura, Commutative ring theory, Translated from the Japanese by M. Reid, Cambridge Univ. Press, Cambridge, 1986.

[Mk07] K. Matsuki, Resolution of singularities in characteristic zero with focus on the inductive algorithm by Villamayor and its simplification by Włodarczyk. preprint, formally a revision of math.AG/0103120, but completely rewritten from scratch, 2007.

[Moh92] T. T. Moh, Quasi-canonical uniformization of hypersurface singularities of char- 
acteristic zero, Comm. Algebra 20 (1992), no. 11, 3207-3249.

[Moh96] T. T. Moh, On a Newton polygon approach to the uniformization of singularities of characteristic $p$, in Algebraic geometry and singularities (La Rábida, 1991), 49-93, Progr. Math., 134, Birkhäuser, Basel.

[Nag57] M. Nagata, Note on a paper of Samuel concerning asymptotic properties of ideals, Mem. Coll. Sci. Univ. Kyoto. Ser. A. Math. 30 (1957), 165-175.

[Nar83a] R. Narasimhan, Hyperplanarity of the equimultiple locus, Proc. Amer. Math. Soc. 87 (1983), no. 3, 403-408.

[Nar83b] _ Monomial equimultiple curves in positive characteristic, Proc. Amer. Math. Soc. 89 (1983), no. 3, 402-406.

[Oda73] T. Oda, Hironaka's additive group scheme, in Number theory, algebraic geometry and commutative algebra, in honor of Yasuo Akizuki, 181-219, Kinokuniya, Tokyo.

[Oda83] $\longleftarrow$, Hironaka's additive group scheme. II, Publ. Res. Inst. Math. Sci. 19 (1983), no. 3, 1163-1179.

[Oda87] _ Infinitely very near singular points, in Complex analytic singularities, 363-404, Adv. Stud. Pure Math., 8, North-Holland, Amsterdam.

[Par99] K. H. Paranjape, The Bogomolov-Pantev resolution, an expository account, in New trends in algebraic geometry (Warwick, 1996), 347-358, Cambridge Univ. Press, Cambridge.

[Pil04] O. Piltant, Applications of ramification theory to resolution of three-dimensional varieties. A talk at the "Workshop on resolution of singularities, factorization of birational mappings, and toroidal geometry" at Banff International Research Station, Banff, during 11-16 December, 2004.

[Pom74] M.-J. Pomerol, Sur la strate de Samuel du sommet d'un cône en caractéristique positive, Bull. Sci. Math. (2) 98 (1974), no. 3, 173-182.

[Spi04] M. Spivakovsky, Puiseaux expansions, a local analogue of Nash's space of arcs and the local uniformization theorem. A talk at the "Workshop on resolution of singularities, factorization of birational mappings, and toroidal geometry" at Banff International Research Station, Banff, during 11-16 December, 2004.

[Tei03] B. Teissier, Valuations, deformations, and toric geometry, in Valuation theory and its applications, Vol. II (Saskatoon, SK, 1999), 361-459, Amer. Math. Soc., Providence, RI.

[Vil89] O. E. Villamayor, Constructiveness of Hironaka's resolution, Ann. Sci. École Norm. Sup. (4) 22 (1989), no. 1, 1-32.

[Vil92] _ Patching local uniformizations, Ann. Sci. École Norm. Sup. (4) 25 (1992), no. $6,629-677$.

[Vil06a] L Rees algebras on smooth schemes: integral closure and higher differential operators. preprint, http://arXiv.org/abs/math.AC/0606795, 2006.

[Vil06b] _ Hypersurface singularities in positive characteristic. preprint, http:// arXiv.org/abs/math. AG/0606796, 2006.

[Wło05] J. Włodarczyk, Simple Hironaka resolution in characteristic zero, J. Amer. Math. Soc. 18 (2005), no. 4, 779-822 (electronic).

[Wło07] _ Giraud maximal contact in positive characteristic. in preparation, 2007.

[Zar40] O. Zariski, Local uniformization on algebraic varieties, Ann. of Math. (2) 41 (1940), 852-896. 